\title{
Efficiency and Market Share in Hungarian Corporate Sector
}

By: László Halpern and Gábor Körösi

Working Paper Number 333

July 2000 


\title{
Efficiency and Market Share in Hungarian Corporate Sector ${ }^{\dagger}$
}

\author{
by
}

\author{
László Halpern* and Gábor Körösi**
}

\begin{abstract}
One of the major tasks facing a transition economy is to create the competitive environment of a properly functioning market economy. It is widely believed that competition has positive effect on efficiency, but the theoretical and empirical support is quite scarce. The objective of this paper is to investigate the link between competition and efficiency for the Hungarian corporate sector during various phases of the transition process. We employ frontier production functions for exploring differences among groups of firms, and for identifying the typical adjustment process of each group separately throughout the transition period until 1997. Groups are defined according to industries, size, and ownership.

The estimated production functions indicate a gradual improvement in efficiency and a shift from decreasing to increasing returns to scale due to growing share of small firms entering higher returns regime. Market share can be explained by the degree of internal and external competition and by the efficiency of the firm.

Transitional recession in 1990-1 was followed by a fast consolidation period, with rapidly increasing firm level efficiency and improving returns to scale. This consolidation period ended in 1994-5, after that mean firm level efficiency only changed slowly. Massive investments largely increased the market share of the better performing firms and sectors, resulting in rapid economic growth. However, this economic growth may become vulnerable if productive efficiency fails to improve faster.
\end{abstract}

JEL Classification: C23, D21, D24

Keywords: Firm in transition economy; production functions; efficiency

$\dagger$ The financial support of EU Commission PHARE-ACE programme No. P97-8131-R and of OTKA No's T 019698 and T 029153 is gratefully acknowledged. We thank Loránd Ambrus-Lakatos, David Brown, Wendy Carlin, John Earle, Réka Horváth, Miklós Koren, László Mátyás, and Jan Svejnar for their comments and suggestions to earlier versions of this paper.

* Institute of Economics of Hungarian Academy of Sciences, CEPR, WDI and CEU, Budaörsi 45, Budapest 1112, Hungary, Tel: +361-3092661, Fax: +361-3193136, email: halpern@econ.core.hu

** Institute of Economics of Hungarian Academy of Sciences and CEU, Budaörsi 45, Budapest 1112, Hungary, Tel: +361-3092671, Fax: +361-3193136, email: korosi@econ.core.hu 


\section{Introduction}

Hungarian firms have gone through different periods of economic transformation since the liberalization of prices and imports in 1988-9. Initially, most firms just waited and did not adjust their capacities to the fall in internal and external demand. Although many (usually small) private firms were established in the period 1988-91, the privatisation of state-owned enterprises (SOE's) just started. The increased competition from both newly emerging firms and from liberalized imports, and more importantly, the loss of the former CMEA markets led to a severe recession and to a deep crisis of the banking sector in 1992-3. The adoption of new accounting standards and a tough bankruptcy law in 1992 contributed to the acceleration of restructuring, which was further enhanced by substantial foreign investment and the emergence of private firms. The March 1995 stabilization measures intended to re-establish the macroeconomic equilibrium in current accounts and in the general government budget and also to shift revenues to the corporate sector. As the privatisation, helped by the inflow of foreign capital, progressed, it created a favourable environment for better corporate performance.

Figure 1 clearly reflects the macroeconomic consequences of this process: The crisis in 1990-1 was followed by a gradual recovery, first in productivity ${ }^{1}$, from 1994 also in GDP growth. In this paper we are mostly concerned with developments in Hungarian corporate sector, underlying this gradual improvement.

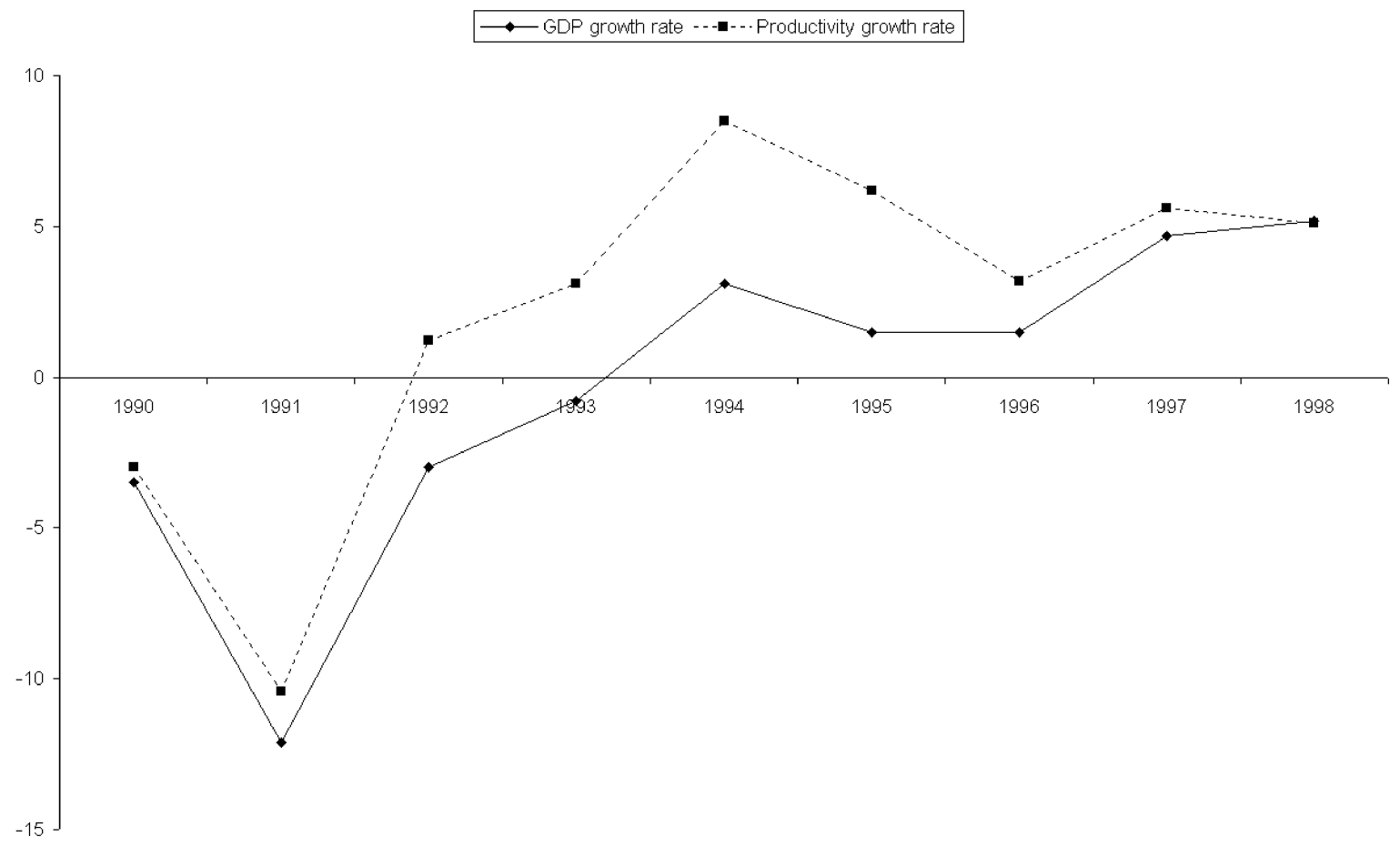

Figure 1. GDP and productivity, annual change in percentages

$\overline{1}$ Productivity is measured here as GDP over employment. 
This work was largely motivated by our earlier study of the performance of large Hungarian exporters (Halpern and Körösi (1998b)), where we basically analysed the factors determining the profitability of these firms, but we also estimated production functions. The results from the estimated production functions were very difficult to reconcile with other findings. Those estimates were average production functions, thus, we implicitly assumed that firms used the factor inputs efficiently, which is an unlikely proposition for firms undergoing serious restructuring. Our maintained hypothesis in this study is that most firms operate far away from the efficiency frontier during transition: partly because they underutilize existing capacities due to the lack of demand, and partly because many firms operate rather inefficiently during the reorganization period. Our former estimates may have been severely biased due to these circumstances. Halpern and Körösi (1998c) was our first attempt to overcome these problems; this paper revises and extends the analysis therein.

This study tries to assess the development of corporate performance between 1990 and 1997 on a larger, more comprehensive sample. Dynamic Cobb-Douglas frontier production functions were estimated. Frontier production functions can directly take into account the above inefficiencies. Dynamic functions provide estimates to assess the speed of the adjustment process to the new, changed environment of the firms. The estimated inefficiencies were subsequently used to explain the development of market share, together with import penetration and concentration. For this purpose balance sheet and profit and loss account data of a sample of several thousands of firms were used. Different subsamples were defined and analysed along sectors, size, and ownership.

The remainder of the paper is organized as follows. Section 2 provides some background on former studies of corporate performance. Section 3 discusses data issues. The framework of our empirical analysis is set out in Section 4. Empirical findings are analysed in Section 5. Finally, conclusions are drawn.

\section{Corporate performance}

The analytical framework of this study was largely set out in papers by Nickell (1996), Nickell et al. (1997), and Hay and Liu (1997). It is assumed that corporate efficiency is closely related to the structure of the market, prices, and firms' costs, hence profits may depend on the degree of competition. In this respect one can distinguish two approaches. In the first one, corporate cost level is outside the control of the firm. Their survival depends on the degree of competition and on the cost level of the rivals. According to the second interpretation cost level is a negative function of efforts, managerial and investment activities. Adopting the second approach the results of the effort of each firm can be compared with that of the best- practice firm and the relative efficiency can be assessed. According to the assumptions this (in)efficiency affects the market share and can be related to other performance indicators. It is, however, obvious that the relation between these categories may be simultaneous. The relation between efficiency and profitability, or investment activity may also be simultaneous, and only an empirical investigation may shed light on its nature. 
In principle one can distinguish between short- and long-run changes in efficiencies. Long-run efficiency can be influenced by the adoption of new technologies, by investment, while short-run efficiency depends on the ability of the management to allocate the existing capacities optimally according to market conditions. If the dynamics of these efficiencies can be assessed, then the time profile of performance indicators can be separated.

It is important to incorporate the basic market characteristics into the model. The more competitive the market, the stronger the link between efficiency and market share, i.e., in a very competitive market only the efficient firms have a good chance for survival. In a less competitive environment less efficient firms can also survive, and the relationship between efficiency and market shares will be weaker.

The speed and degree of price and foreign trade liberalization, the rules and costs of entry and exit for domestic and foreign participants, influence the development of markets in transition economies. They are quite different across countries. However, low capacity utilization, the increasingly large number of market participants, the lack of legal, behavioural and institutional stability and of transparency are common characteristics of transition economies, distinguishing them from mature market economies.

Hungarian corporate sector attracted substantial foreign direct investment compared to other transition economies. Large number of new firms was created, partly as spin-offs of liquidated firms. New domestic and foreign firms are assumed to be leaders of the competition, to be more efficient than the others. These assumptions will be investigated.

Similar investigations were made by Brada et al. (1997) for Hungary for 1991 and for Czechoslovakia for 1990, and by Konings and Repkin (1998) for Bulgaria for 1993-5 and Romania for 1994-5 and recently by Brown and Earle (2000) for Russia. Our results are not, however, directly comparable to these studies. There are major differences in the model specification, and also in the sample period. Nevertheless, the main direction of these studies is similar to ours as the estimation of frontier production function is concerned. This paper goes beyond the scope of these studies as the aim of this paper is the investigation of the relationship between efficiency and market share. The behaviour of Hungarian firms, the link between performance and ownership have been analysed by other studies (c.f., Major (1999) and Tóth (1999)), but none of them aim at assessing the link between performance and market power.

\section{Data}

The database for this empirical study consists of the profit and loss account and balance sheet data of the main Hungarian firms between 1989 and $1996 .{ }^{2}$ This dataset is linked to another database: a labour market survey database, although the latter is not used in the present study. ${ }^{3}$

2 We would like to express our gratitude to Mr. József Becsei and his collaborators for their help in compiling the data base.

3 We plan to extend the analysis by resolving the labour homogeneity assumption. In the labour market survey employment is differentiated by occupational categories and educational attainment. 
The corporate dataset covers those firms which were present in the labour survey. The survey theoretically covers all firms with at least 20 employees, but the actual compliance is far from complete, especially among smaller firms. On the other hand, some smaller firms, employing fewer people, also are in the sample. ${ }^{4}$ However, the corporate dataset also includes data in the 'neighbouring' years, if the firm could be identified for those years. That is, if a firm only participated in the labour survey in 1993, our dataset should include the balance sheet of the firm in years 1992-94, provided that the firm existed and following up the firm was possible.

Firms are identified by their tax-file number in the dataset. If a firm was reorganized: broken up, merged with another firm, or, sometimes, it simply changed name, relocated headquarters, etc., it got a new tax-file number. As our sample covers the period, when former SOE's were corporatised, frequently reorganized, and later privatised, there were many such changes, when a new tax-file number had to be assigned to the firm. Thus, in some cases, existing firms disappear from our sample, because their tax-file number was changed for some reason, and 'new' firms enter the dataset where the tax-file number is the only novelty. Unfortunately, we cannot distinguish de novo firms from the reorganized ones, or those split off from existing firms. Both groups are rather large: Since the mid1980's many small private firms were started. Many grew to considerable size, and they represent an important fraction of new firms in our sample, although they frequently enter this sample after several years of operation. On the other hand, there are many new firms created from existing former SOE's: Voszka (1997) reports that from remnants of 49 well-known former socialist SOE's which in 1989 produced approximately $30 \%$ of Hungarian GDP and 50\% of the exports at least 690 firms were created by 1996, most of them privately owned by then.

Our classification is further hampered by the fact that existing firms did not always participate in the labour survey for the entire sample period, thus, the firm may have been incorrectly classified as new or disappearing. Firm creation and destruction is overreported in our database, and thus in our analysis for all these reasons.

As the corporate dataset is a mirror image of the labour survey, sample selection is biased towards large firms. Only those firms are covered which have to comply with double-entry accounting rules, thus family firms and individual entrepreneurs are excluded, unless their equity or turnover exceeds a rather high limit. The dataset covers approximately $10-13 \%$ of the incorporated firms in each year. The sample included 2682 firms out of 23314 in 1990 and 11172 firms out of 120423 in 1997. The coverage varied a lot over sectors: while only $5-7 \%$ of trading firms are included, coverage is over $50 \%$ in mining in all years. The sample almost always covers at least $20 \%$ of the firms in all broad industrial sectors.

The coverage is, however, much higher with respect to sales volume. It is more than $50 \%$ even in the trade sector. In other sectors, including services and agriculture, at least $70 \%$ of the sales were at firms included in our sample. There are sectors, like mining, or electricity generation, where the coverage is well over $90 \%$.

Many observations, however, had to be excluded due to data problems, e.g., missing observations, so the actual sample size of the estimations is smaller, but the coverage,

$\overline{4}$ Before 1992 agriculture and some service sectors were excluded from the survey. 
measured by sales, is still high in all years. There was one important characteristic feature of the sample, which has a strong systematic influence on our results: There are many firms with negative (or zero) value added. As the dependent variable of the reported production functions is the logarithm of the value added, these firms had to be excluded from estimation. These firms represented more than $5 \%$ of our sample in all years, peaking with $20 \%$ of the covered firms in 1991 . These firms are the heavy loss makers, frequently bankrupt or at least approaching insolvency. Some resurface in later years, but most of them were closed down. This characteristically different group of firms was excluded from the current analysis, although we plan to study them later. ${ }^{5}$

Capital is a key variable of production functions. It is always difficult to measure capital stock appropriately. It is a probably even more problematic task in a transition economy. The assets of practically all pre-existing firms were revalued at least once (frequently for several times) during the process of commercialisation and privatisation. The asset value could change substantially without any change in the physical composition of the capital, and the timing of the revaluation(s) is unknown. For example, in the 1992 sample some firms will have capital stock recently revalued, and it is supposed to reflect the actual market value of the assets. Other firms, where no reorganization occurred, reported assets calculated from past investments flows. That certainly influences our results, however, we cannot assess its importance.

Definitional changes also caused some problems. Some definitions changed with the introduction of new accounting standards in 1992, but those changes could be followed through. Sectoral classification also changed in 1992. We aggregated the sectoral classification to a level where it is reasonably homogeneous for the sectoral subsamples, but some inconsistencies are inevitable. The four digit sectors, used for the determination of the market size, substantially changed from 1991 to 1992.

\section{Estimated Models}

The starting point of our analysis is the traditional Cobb-Douglas production function in its linearized form. We assume that the production function describes the potential production of the firm, thus, we use frontier production functions. We follow the traditional approach first suggested by Aigner et al. (1977):

$$
\log \left(Y_{t, i}\right)=c+\alpha \log \left(L_{t, i}\right)+\beta \log \left(K_{t, i}\right)+\gamma \log \left(Y_{t-1, i}\right)+v_{t, i}-u_{t, i},
$$

where $v$ is the usual disturbance term (assumed to be $v \sim \mathcal{N}\left(0, \sigma_{v}\right)$ ), while $u$ is assumed to have truncated normal distribution (for $u>0$ ), representing firm specific inefficiencies, compared to the 'best-practice' firm in the sample. As the returns to scale may also be interpreted as a measure of allocative efficiency of input use, or of market imperfection, we did not impose constant returns to scale (CRS).

5 We experimented with production functions, where the dependent variable was sales. These estimates were severely influenced by the observations corresponding to firms with negative value added: The overall performance of the production functions, estimated for the entire sample was worse than those estimated for firms with positive value added only, and the likelihood function was also much worse conditioned. 
The lagged dependent variable captures the fact that with substantial changes in factor input or in circumstances adjustment to the new long-run production level may take a relatively long time. Fixed time effect is also included in all panel estimates, which in this case represents the change of the mean (in)efficiency for that year compared to the (first) base year.

The difference with respect to the best-practice firm is defined as inefficiency. ${ }^{6}$ There are at least two possible problems with this interpretation. First, this measure is a general capacity disutilization. In both market and emerging economies capacity (dis)utilization can be different across factors which is not allowed in our specification. The other possibility is that labour and/or capital are not homogenous, labour skills and capital might be different across firms or sectors. Our present approach is not appropriate for choosing between these interpretations. Otto (1999) attempts to separate them at the aggregate level.

Frontier functions were estimated in two forms. First, the functions were augmented by variables reflecting the competition firms have to face. Three variables are used to describe this pressure: import penetration, concentration and market share. We expect positive coefficient for import penetration, market share and negative for concentration. The rationale behind is that stronger competition may force the company to become more efficient. Market share is lagged in order to avoid possible simultaneity: More efficient firms may increase their market share, thus leading to a possible reverse causality. On the other hand increasing market share may be associated with weakening competition. Second, a 'simple' production function was coupled with a dynamic second equation, describing the market share of the firm which included the same indicators of competitive pressure (concentration and import penetration) and also the residual $\widehat{u}$ of the production equation, representing the efficiency of the productive process. ${ }^{7}$ The market share equation:

$$
\text { share }_{t, i}=\gamma_{0}+\gamma_{1} \text { share }_{t-1, i}+\gamma_{2} \widehat{u}_{t, i}+\gamma_{3} \operatorname{conc}_{t, i}+\gamma_{4} i m p p_{t, i}+\epsilon
$$

The underlying assumption is that efficient firms will gain market share. We expect that efficiency has a positive and growing effect on market share as long as market institutions evolve and competition increases. We also assume that concentration has a positive effect on market share, as higher concentration is associated with a less competitive market making it easier to increase market share. Finally, the import penetration is expected to enter the equation with negative sign, since higher import penetration increases domestic competition and reduces market share for domestic firm.

We also check the hypothesis that profitability may be related to efficiency and that investments may influence efficiency. Simple linear correlation is used for this purpose.

The models used throughout this paper are best applied for manufacturing. One important feature of the Hungarian corporate sector is that the sectoral classification

6 Due to the features of the data set it was impossible to estimate a panel model with fixed firm effect to separate short and long term inefficiencies.

7 Hay and Liu (1997) found for UK data that efficiency is exogenous to the market share. The reverse causation was also examined, long run efficiency was regressed on investment, short run efficiency was explained by lagged market share, lagged gross profit and by rival firms efficiency. Due to data constraint we were unable to explore all these issues. 
may be biased, the principal activity of the time of registration may be totally different of the actual one and firms are following quite distant and heterogeneous activities. That is why results for non-manufacturing sectors were also analysed.

\section{Estimation results}

We estimated the outlined models for the entire sample, and also for various subsamples. The equations were estimated for sectors; for small, medium-sized, and large firms; and for five ownership categories. The sectoral classification of this study is: Agriculture, Manufacturing, Construction, Trade, and Services. Within manufacturing Engineering, Chemical industry, Food industry, Light industry, ${ }^{8}$ and Other industries were distinguished. Observations were grouped for state-owned, domestic private, foreign, important foreign minority and other ownership categories. ${ }^{9}$ We do not report estimation results for all subsamples. However, for most subsamples for most years we got significantly different coefficient vectors than for the entire sample. Thus, the behaviour of firms is not homogeneous. These structural differences necessitate the analysis of characteristic differences over the various groups of firms.

The frontier production function is relevant only if the second component of the disturbance term, representing (in)efficiency, is different from 0 . This is indicated by the ratio of the two standard errors $\left(\sigma_{v} / \sigma_{u}\right)$. There is a small number of cases, when the estimation of this ratio converges to extremely low (or large) values. This obviously indicates severe specification error in those cases involved, however, we were unable to find a consistently better specification. ${ }^{10}$ In most cases the ratio of the two standard errors in the equation was above unity. These estimates are significantly larger than the usual estimates for developed countries. It may reflect higher inefficiency of firms in Hungary, compared to developed market economies, although direct comparison is strongly influenced by the actual model specification, the characteristics of the sample information, and also by the variance of the traditional disturbance term. Anyway, the overwhelmingly significant estimate for this coefficient clearly indicates that the use of frontier production functions was justified.

Tables 1-3 present results of panel estimates for the single equation model, while Tables 4-6 the same for the two-equation one. The same models are estimated for the entire period, for two sub-periods (1990-3 and 1994-7) and for biannual panels. Parameter estimates in different time periods are significantly different from each other according to the structural break tests, 1996-7 excepted. The two four-yearly panels have very different estimated properties. The four-yearly and the biannual panel estimates suggest very

8 Light industry consists of textile, clothing, leather, footwear, wood, paper and printing industries.

9 The definitions of size and ownership categories are given in Appendix A, while estimation results in Appendix B.

10 Other distributions were also tried (c.f., Greene (1993) for further details), however, the attempted other distributions usually also led to very questionable estimates. The convergence problems usually emerge when the sample size becomes relatively small. As ill-conditioned likelihood function can just be the consequence of small sample size, we did not want to present estimation results from a different specification for these cases. 
different time paths for the change in mean inefficiency for 1990-3. These discrepancies are much smaller for the second half of the sample period, but these panel estimates may still be biased.

Even though coefficient vectors, estimated for the later years, are not far from each other, the significant structural breaks for most of the sample period suggest that sample information should be treated as a series of repeated cross-sections instead of a single panel. Thus, we continued our analysis by departing from the panel framework. Sample information is as repeated cross sections of a large and growing number of heterogeneous firms. Tables 7-10 B summarize estimation results for each year, ${ }^{11}$ while Tables 11-27 present estimates for relevant groups of firms: classified according to sectors, ownership and size.

There are two models for each sample: production function augmented with market variables, and a two equation model consisting of the production and the market share equations. Both models have high explanatory power, but the market share equation of the second model is usually far from being satisfactory: residuals are heteroscedastic, and the reset test indicates incomplete specification. Estimated coefficients are in line with the expectations with some exceptions: The sign of import penetration is uncertain, and concentration is usually insignificant in the single equation model. These variables perform better in the two-equation model.

The overall picture on the corporate performance is, that the 1990-1 transitional crisis was characterized by huge inefficiencies and decreasing returns. Corporate efficiency improved rapidly from 1992, also accompanied by higher (close to constant) returns to scale, or, from 1994 even slightly above that. However, firm level efficiency improvement was substantially slower from 1995. It was no longer uniform: the heterogeneity of the firms increased with respect to efficiency, but this increase cannot be attributed to any specific group of firms.

There are, however, interesting differences behind the general tendencies, which will be analysed in the following sections. First, the analysis of the efficiency of the production process is presented. Second, the market share equations and the role of the variables representing competitive pressure are discussed. Third, the link between efficiency and profit and investment is shown. Fourth, returns to scale estimations according to industries and size are presented.

\subsection{Efficiency}

One can look at the mean (in)efficiency of the production process within a group of firms in two alternative ways. On the one hand, when the production function is estimated for the entire sample of all firms, groups of firms (say sectors) can be ranked according to differences in the mean efficiency. This is the traditional interpretation, and in this paper we mostly deal with this efficiency measure. On the other hand, when the production function is estimated for the individual groups of firms, mean inefficiency of the group

\footnotetext{
11 The $B$ tables consist of mean inefficiencies of various group of firms. While ranking firm level inefficiency would be a futile effort, these means have relatively small variances, thus their comparison is meaningful.
} 
reflects the heterogeneity of firms with respect to efficiency. Theoretically, the two measures may develop very differently: It may happen that a sector is rather homogeneous, all firms are close to the most efficient one within the sector, thus the mean inefficiency of the sectoral production function is small. But that does not tell anything about the efficiency of the sector, compared to other sectors: It may happen, that the overall efficiency of the production process is (uniformly) much lower in this sector than in others. It is interesting to note that these two sorts of efficiency measures developed rather similarly over time and over the relevant groups of firms in the Hungarian corporate sector. It indicates that specific groups usually had lower or higher overall mean efficiency because firms were more or less heterogeneous within the group. We look at the overall efficiency of firms from three different aspects in the following subsections: Sectoral differences, and variations according to the size and ownership of the firms.

Fixed time effects of the panel estimates reflect year to year changes of the mean inefficiency, compared to the base year. Although conflicts emerge between estimates for panels covering different time periods, their overall pattern is similar to the time path emerging from cross-section estimates. This clearly indicates that very powerful forces shaped the productive efficiency of Hungarian firms during the transition period; no matter how we look at it: in panel models, at aggregate level, or for various groups of firms, we get similar results. Thus we do not analyse these panel estimates separately.

\subsubsection{Sectors}

The time path of the efficiency of various sectors is quite similar to the overall picture obtained from the panel estimates: Substantial drop in 1991, rapid growth until 1994 and a mild decline afterwards. (See Figures 1 and 2.) There is a curious discrepancy between this assessment of the developments in corporate efficiency and the aggregate (macroeconomic) development: Economic growth was rather sluggish after the 1991-2 recession, and it speeded up after 1996, by which time the productive efficiency of the corporate sector did not improve. It indicates that the substantial deterioration of firm level efficiency in 1991 basically reflected the enormous capacity underutilization of most firms, due to the sudden loss of important markets. First, firms had to adjust their capacities to the realities of the new market conditions, probably cutting excess capacities, and they could efficiently use the productive inputs only afterwards.

While practically all major sectors move to the same direction, important sectoral differences can be observed. Agriculture is usually the least efficient sector, and it is clearly left behind by other sectors at the end of the sample period. Services and Manufacturing are the most efficient sectors in the second half of the sample, although these differences are only noticeable in the dynamic specifications.

The picture is somewhat different within Manufacturing; there is no decline after 1994 for Engineering and Light industry. The 1991 crisis hit Engineering the hardest, but it recovered within two years, and it became the most efficient industry. Pharmaceuticals, the traditional standard bearer in the Hungarian corporate sector, on the other hand, suffered a major efficiency loss in 1993, and it no longer stands out. 


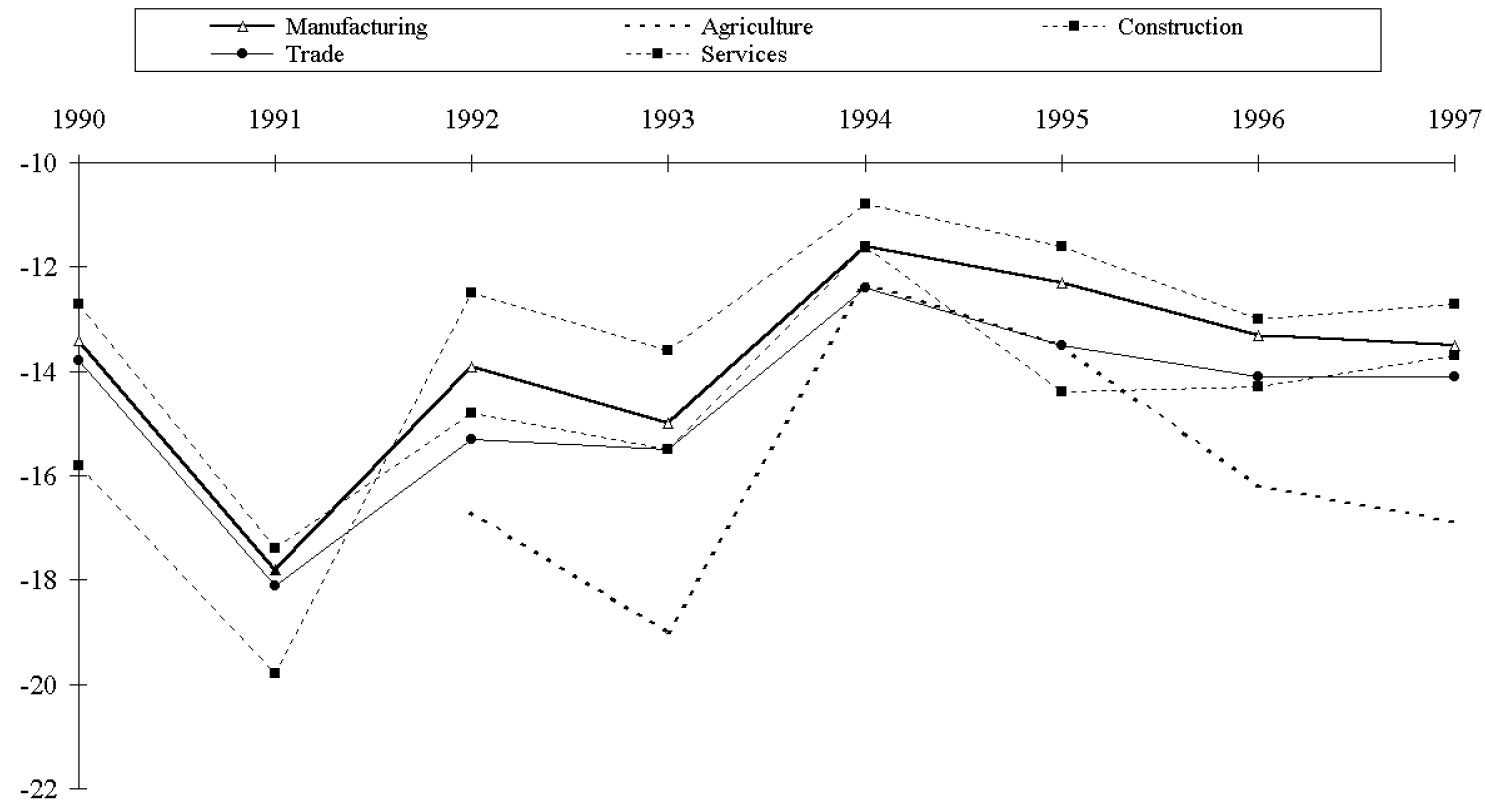

Figure 2. Sectoral mean inefficiencies

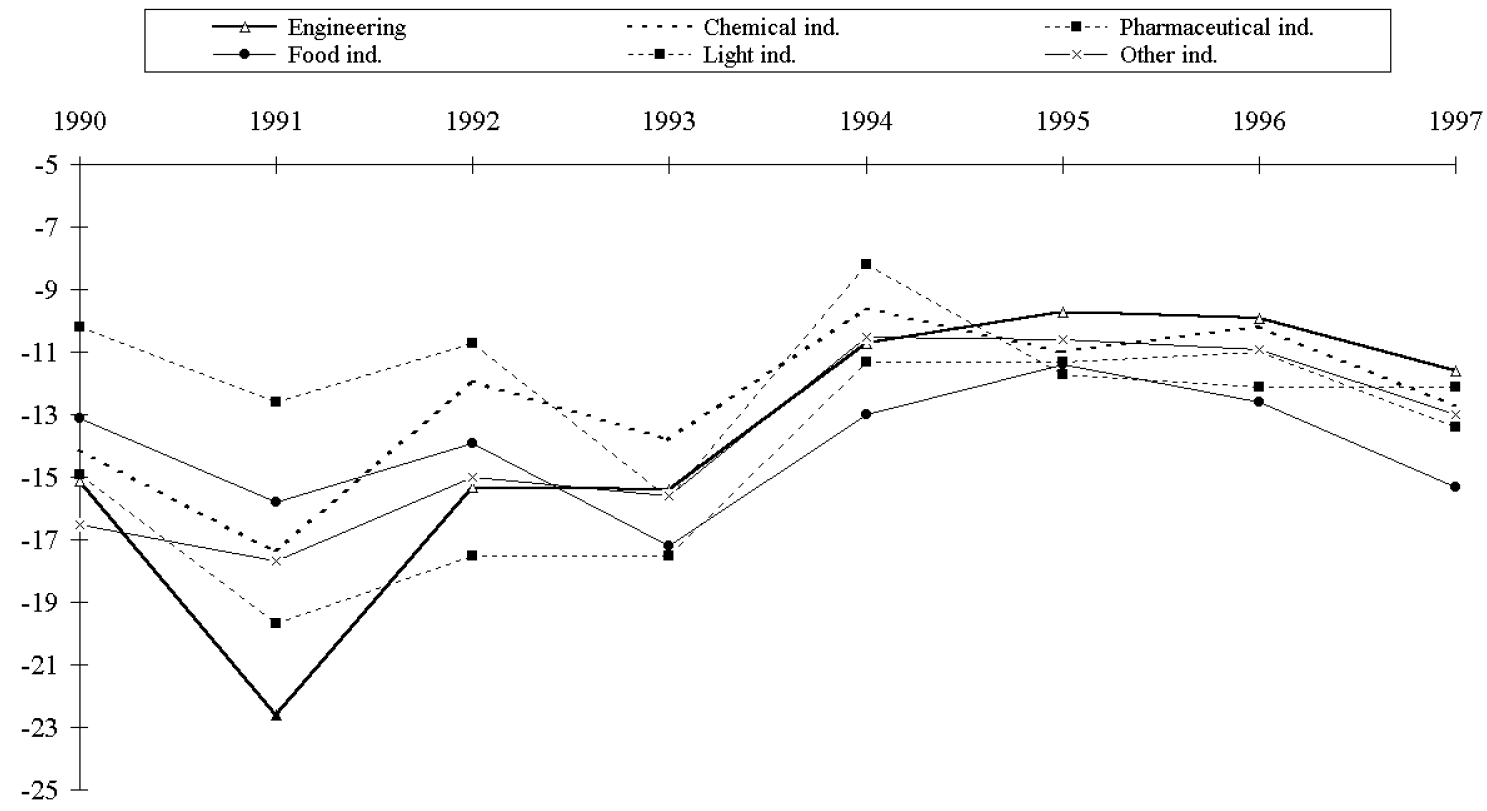

Figure 3. Mean inefficiencies in the manufacturing sectors 


\subsubsection{Size}

Three size groups were defined: small, medium and large. (See definitions in the Appendix). Results derived from the estimation on the entire sample reveal that large firms were consistently the most efficient group. (See Figure 3.) The difference between the other two groups was negligible. This ranking is also supported by the individual estimation results for these groups, and the heterogeneity decreases with size. There are curious discrepancies between these efficiency estimates and the returns to scales, to be analysed later.

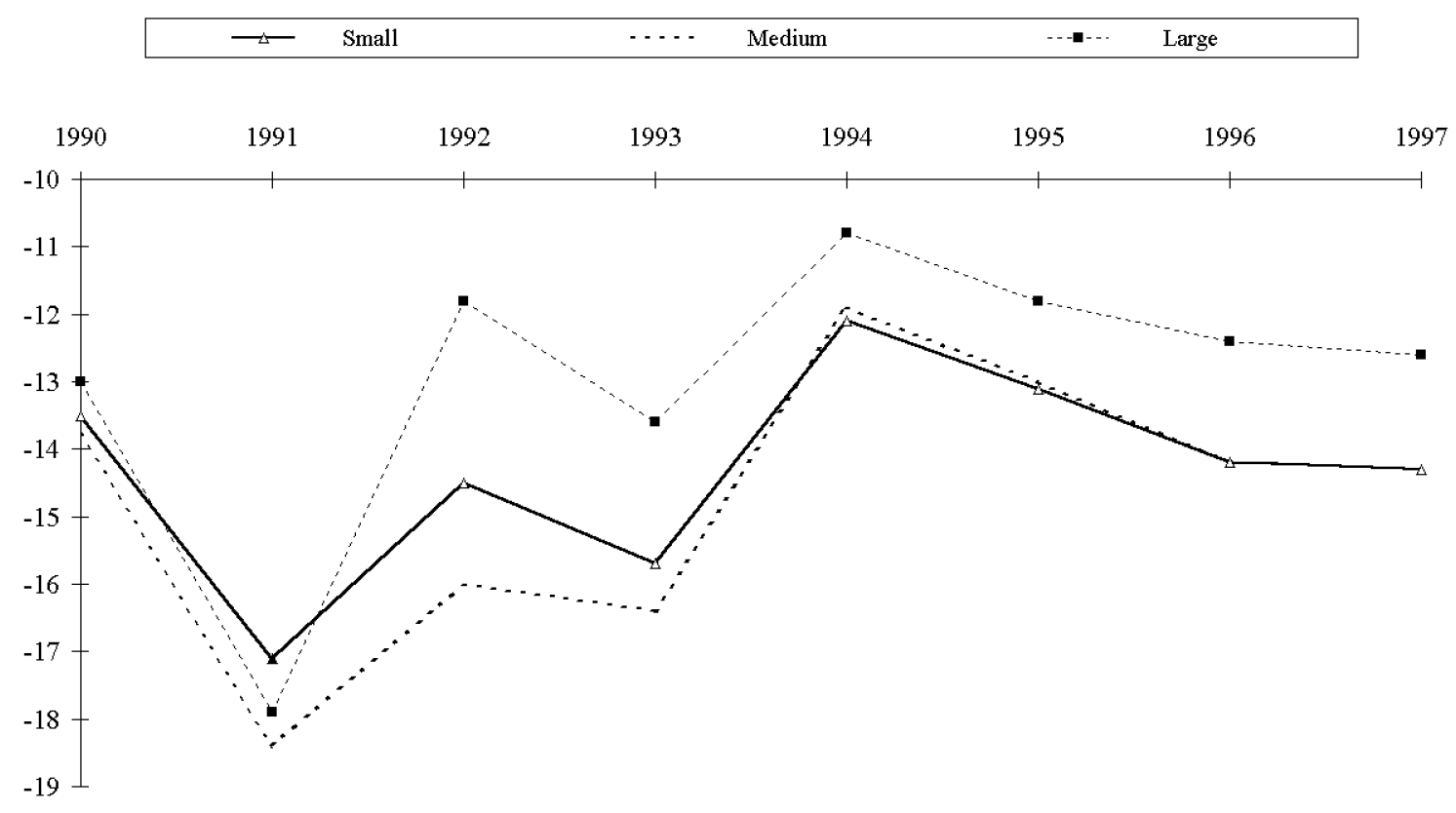

Figure 4. Mean inefficiency by size

\subsubsection{Ownership}

There is a clear ranking in efficiency according to ownership: 1) foreign 2) important foreign 3) domestic private 4),5) other and state. It is true for the entire sample and even more pronounced for Manufacturing. (See Figure 4.) This persistence in ranking can be a result of selection bias in privatisation; it goes beyond the scope of this paper to address the endogeneity issue between privatisation and efficiency. However, we believe that sample selection bias may only be substantial in the initial years: Foreign and domestic private ownership became so widespread after 1993 that persistent substantial differences in the preconditions are unlikely. The more plausible explanation is that these differences are caused by differences in corporate governance, the quality of management, access to markets and resources, etc. The difference between the efficiency among firms in foreign 


\begin{tabular}{lllll|}
\hline$\longrightarrow$ & Private & $\cdots \cdots$ & Government & $\cdots$ \\
Imp. foreign & $\cdots$ & Other & Foreign \\
\hline
\end{tabular}

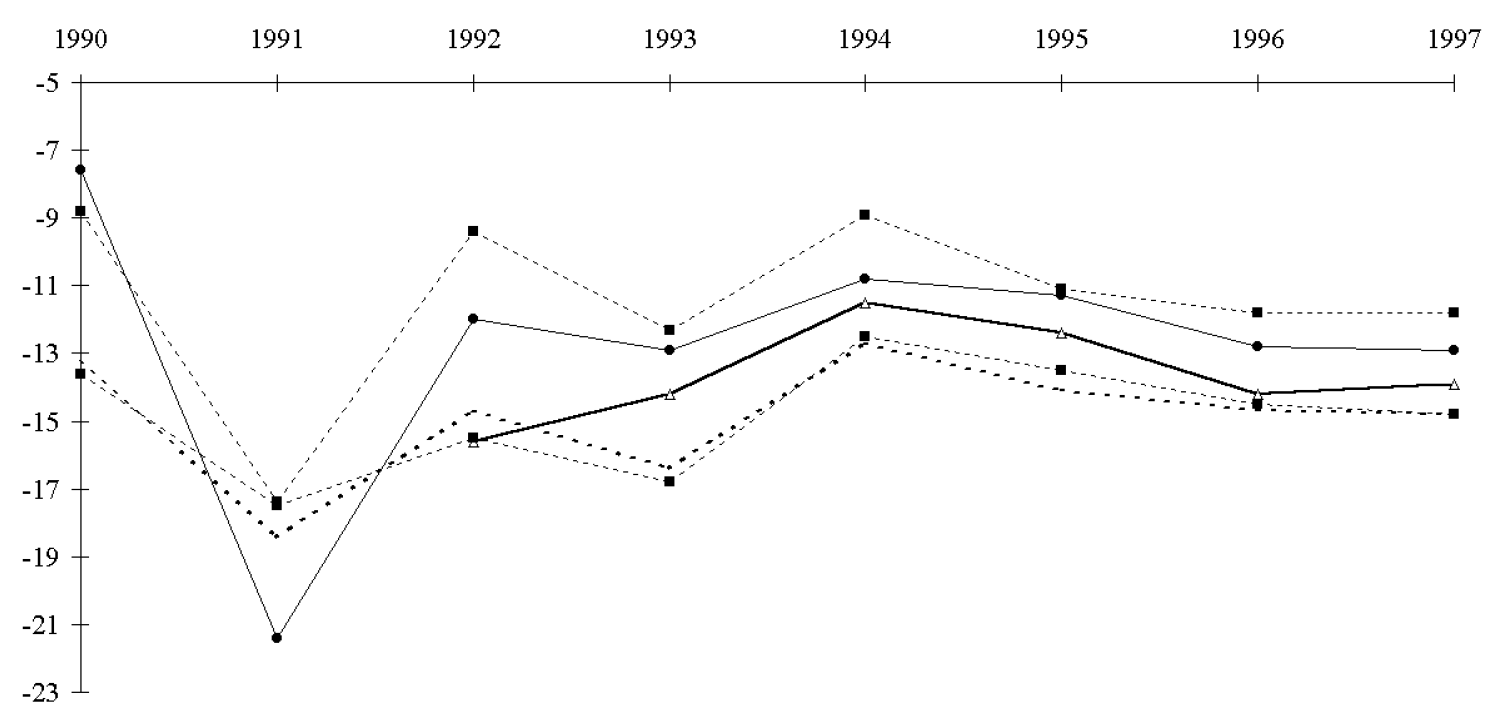

Figure 5. Mean inefficiency by ownership categories

and domestic private ownership is remarkable. It certainly reflects differences in market access. It may also indicate that domestic private owners are very much constrained at the financial markets. It is curious that state owned enterprises are not much less efficient than domestic private owned firms, although most theoretical works would suggest that. This is especially true for the other ownership group, which largely consists of private firms after 1994. ${ }^{12}$ This group of firms includes many medium sized former SOE's, frequently bought up by the (former) management through limited liability companies. They are clearly less efficient than the majority of the corporate sector. The efficiency gap between the firms privatised to foreign and domestic owners clearly indicate a curious failure of the privatisation in creating a group of domestic owners who can operate efficiently and compete internationally. However, fluctuations and the gap between the most and least efficient groups diminished, especially after 1994, indicating a move towards homogeneity and competition. The efficiency gap between firms owned by foreign and domestic investors is, however, persistent, and almost uniform over various groups of firms by size or sector.

12 The other group includes firms with no dominant owner, or firms which have a dominant corporate owner. This second type is much more numerous. Initially the corporate owners usually were state owned holding companies, but as privatisation progressed, the overwhelming majority of these indirectly owned firms were in fact (domestic) private. However, we have no exact information on the ownership structure of the parent company. 


\subsection{Market share}

As indicated earlier, we had little success with the attempt to augment the production functions with variables indicating market conditions and competitive pressure. Coefficients are frequently insignificant or they change sign from one year to the other, and the joint effect of the three variables, describing market structure, is usually negligible. Especially, concentration was hardly ever significant in the single equation model.

In the alternative two-equation model we estimated an autoregressive equation for the market share explained by the productive efficiency (measured as $\widehat{u}$ ), import penetration and concentration. These regressions fit reasonably, but diagnostic tests indicate significant specification problems. These variables most probably are insufficient to explain why firms gain or lose market share. ${ }^{13}$ Thus we have to interpret these results with due caution.

Efficiency usually was significant with the expected positive sign, indicating that efficient firms gain market share. With respect to the other two variables results are rather mixed: They do not play important role in explaining market share, and they rarely enter the equation with correct sign significantly. For example, import played a substitution role in the more intense phase of restructuring in manufacturing; while a complementary role has developed and became general in the second half of 1990s.

\subsection{Efficiency vs. profitability and investment}

As sample information did not facilitate the separation of short and long-term efficiencies, our efficiency measure incorporates both. Efficiency should somehow be correlated to the profitability of the firm and to its investment activity. Simple correlation coefficients show that there was a semi-strong positive link between efficiency and profit margin and only a very weak positive relationship between efficiency and investment. The profit relationship has weakened in 1997, while the investment relation became more common.

\subsection{Returns to scale}

The null of CRS was rejected in almost all cases. In the early years of transition all groups of firms faced decreasing returns to scale, indicating substantial mismatch of input use under the new market conditions. Later returns to scale increased, and the long-run returns to scale actually exceeded unity after 1994. This tendency could suggest another interpretation of efficiency: The larger the firm, the better the output to input ratio. One could also interpret this general tendency as the consequence of institutional and behavioural changes: The hardening of the budget constraint has brought about substantial 13 Our concentration indicator is an unfortunately poor measure to assess characteristics of a sector. We
did not have information on all the firms of a sector to compute better indicators. 
improvements in the resource allocation, hence in efficiency. Another important possibility is related to the market structure and entry conditions: The larger the firm, the wider the possibility to have access to monopoly rents. ${ }^{14}$

Market structure can be very different across sectors, resulting in a varying potential for increasing return to scale. However, the sectoral differences were rather small. All sectors started with decreasing returns to scale in 1990-1, and most, except Agriculture and Services, entered the increasing return to scale region by 1997. It is also important to note that the sectoral variation declined a lot.

We also estimated separate models for samples defined by ownership. ${ }^{15}$ The above tendency is true for the ownership classification with two exceptions: SOE's were always in a regime with increasing return to scale, starting from 1992, while firms classified as Other ownership left that regime in 1996 and 1997.

It would be easy to jump to apparently obvious conclusions at this stage, e.g., saying that two major factors contribute to the increasing returns to scale: (i) The sample includes large number of SMEs. The underreporting of output can be much larger than for the inputs for SMEs; (ii) Some industries are rather concentrated, and very small firms are disadvantaged by oligopolistic competition. These general assumptions, however, are rejected by the analysis of the results when splitting the sample by size.

Classification by size revealed substantial and persistent differences. (See Figure 5.) Results for the entire sample seem to be strongly influenced by the change in composition: by the growing share of small firms (from $1 / 4$ to $2 / 3$ ), and by the increase of their returns to scale. Medium-sized firms were practically in CRS after 1991. Large firms, curiously enough, always stayed in decreasing returns to scale with substantial fluctuations.

We also looked at the interaction of size and ownership, using two ownership categories in this case: foreign and domestic. ${ }^{16}$ The tendency of returns to scale becoming gradually larger was practically the same for all small firms, although foreign owned ones tended to have slightly higher returns to scale. However, for medium-sized, and large firms we found that domestic companies had higher return to scale in almost all years than foreign ones.

This result is rather surprising and casts some doubt on the validity of our data. One could think that large firms have decreasing returns to scale because they are too large for the market, and operate at the increasing part of the U shaped cost curve. However, the large foreign owned firms are typically local subsidiaries of multinational companies. It is an unlikely proposition for them. This is a curious finding for which we do not have acceptable interpretation which can also be substantiated from the available sample information. ${ }^{17}$

${ }^{14}$ In our previous works (Halpern and Körösi $(1998 \mathrm{a}, \mathrm{b})$ ) we studied monopoly rent: It had disappeared around 1989-90, during the period of large scale price and import liberalization, and reappeared afterwards.

15 This analysis could only be started with 1992, as the number of privately and foreign owned firms was far too small before that.

16 The owner is domestic, if the share of foreign ownership is less than $50 \%$.

17 Large multinationals may initially start their activity with low value added, because of the high startup costs, and investing into gaining a large share of the newly entered market. But for many of these firms the target is not the Hungarian market; their production is largely exported. And most of them 


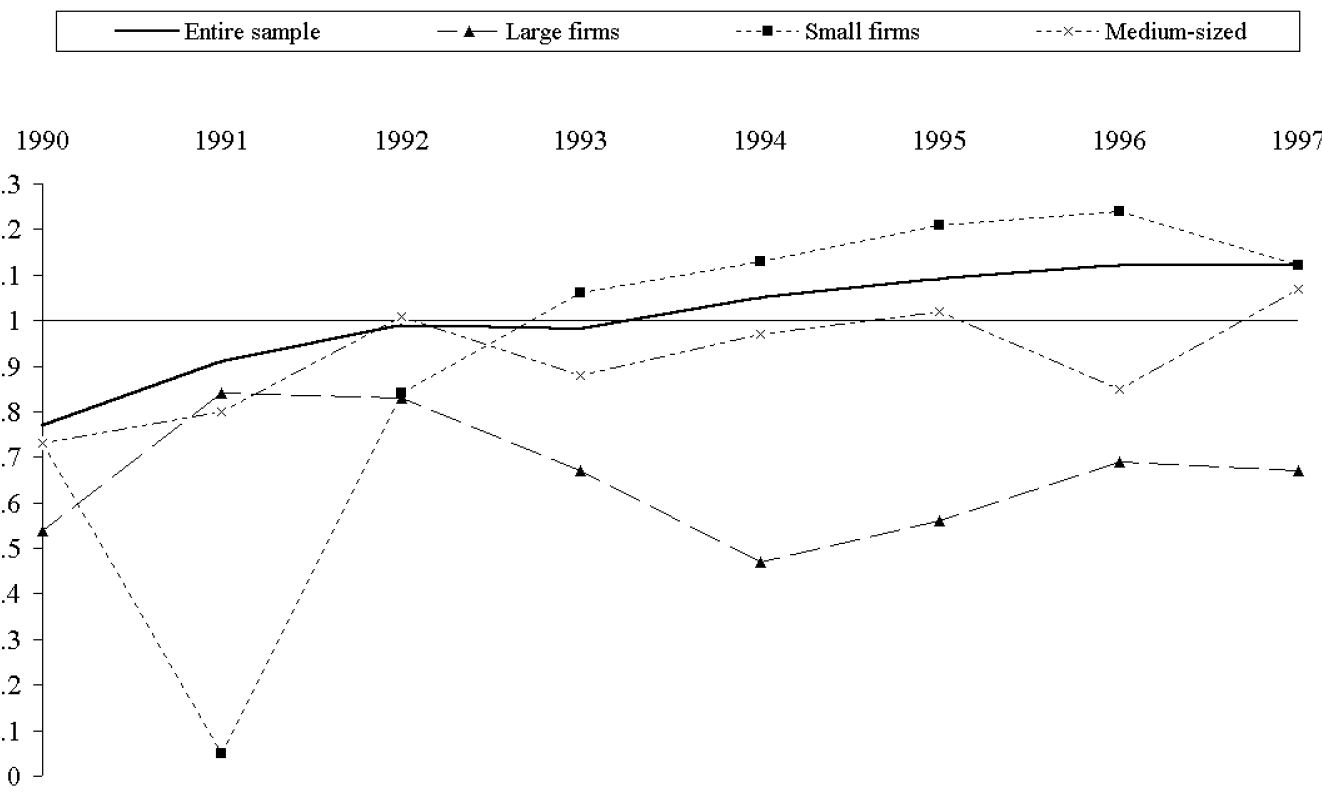

Figure 6. Long-run returns to scale by size categories

Based on these results the market structure explanation of the increasing returns for the entire Hungarian corporate sector should be rejected. The large number of small firms with increasing returns to scale may be interpreted as a positive sign of efficiency and a prospect for further competition. However, it may lead to further differentiation, namely, that very small firms are strongly disadvantaged by their meagre resources and insufficient access to important markets. This possibility requires further investigation.

Given that the returns to scale is smaller for large foreign firms than for any other group, or that one estimated for all firms, the estimated efficiency for this group must substantially understate their efficiency advantage; firms in this group are even more efficient than indicated earlier.

\section{Conclusions}

Our results do strongly qualify the findings of Brada et al. (1997) and of Konings and Repkin (1998), referring to a hypothesis in Ickes and Ryterman (1992), that the larger the firm the higher the allocative efficiency prior to transition. Our results show that there was a substantial difference in efficiency according to size, fluctuations were rather dominated by macroeconomic developments, like the fall in external and/or internal demand, what

entered the Hungarian market rather early, what makes this explanation quite unappealing by the end of our sample period. We do not have data to test a possible explanation: the transfer pricing hypothesis. 
happened in Hungary in 1991 or by a wave of bankruptcies and liquidation in 1993 . Microeconomic restructuring had a positive effect and it can be seen that after 1993 efficiency in different disaggregation became more homogeneous and higher as compared with previous years.

Capacity underutilization is very large in the early years of transition, mainly because of the fall of overall demand and the high cost of supply reaction to the changing pattern of demand. The results for the Hungarian corporate sector between 1990 and 1997 confirm the positive development of the performance after a painful and deep microeconomic restructuring and macroeconomic adjustment. However, the speed and scope of recovery varied substantially over different groups of firms.

State-owned firms were among the least efficient, while foreign-owned firms were clearly the most efficient ones throughout the transition period. This is an important difference to our earlier findings (Halpern and Körösi, 1998a, b) on the performance of firms: The link between profitability and foreign ownership was less obvious, and less persistent, than between efficiency and ownership.

The ability to achieve the highest returns to scale in the relevant market can also be defined as efficiency. Smaller firms seem to perform better than larger ones, what offer two possible explanations: Small firms have used their opportunities better, while large firms, especially foreign ones, either have not been able to perform better what contradicts to previous results on higher efficiency, or did not show up in their data for different reasons. This calls for further investigations.

Market characteristics play a changing role during transition. Import competition, sectoral concentration and efficiency are important explanatory factors for the development of market share of a firm. Heterogeneity can be observed across sectors, according to ownership and to size. The differences, however, are not that large and were diminishing, what makes the hypothesis of the importance of market environment in the determination of corporate performance plausible.

When looking at corporate performance, the 1990-7 period can be divided into three distinct subperiods. Transition started by a sudden collapse of corporate efficiency, as one important element of the transitional recession. It was followed by a fast consolidation period, with rapidly increasing efficiency and improving returns to scale. During this period performance was frequently improved by downsizing, thus fast improving corporate performance could not be translated into economic growth. This consolidation period ended in 1994-5, after that mean firm level efficiency only changed slowly. However, the 1995 stabilization package created a favourable environment for substantial investments into the Hungarian corporate sector. These investments largely increased the market share of the better performing firms and sectors, and the massive investments, together with substantial structural improvements brought about rapid economic growth. However, this economic growth may become vulnerable if productive efficiency fails to improve faster. 


\section{References}

Aghion, P., Blanchard, O.J. and Carlin, W. (1994): The Economics of Enterprise Restructuring in Central and Eastern Europe; CEPR Discussion Paper 1058.

Aigner, D.K., Lovell, K. and Schmidt, P. (1977): Formulation and Estimation of Stochastic Frontier Production Function Models; Journal of Econometrics, Vol. 6, No. 1, pp. 21-37.

Brada, J., King, A. and Ma, C. (1997): Industrial Economics of the Transition: Determinants of Enterprise Efficiency in Czechoslovakia and Hungary; Oxford Economic Papers, Vol. 49, pp. 104-127.

Brown, D.J. and Earle, J.S. (2000): Competition and Firm Performance: Lessons from Russia; CEPR Discussion Paper 2444.

Estrin, S. and Hare, P. (1992): Firms in Transition: Modelling Enterprise Adjustment; Centre for Economic Performance, LSE Discussion Paper No. 89, p. 42.

Färe, R., Grosskopf, S., and Lovell, C.A.K. (1994): Production frontiers; Cambridge University Press, Cambridge.

Greene, W. (1993): The Econometric Approach to Efficiency Analysis; in: Fried, H.O., Lovell, C.A.K and Schmidt, S.S (Eds.): The Measurement of Productive Efficiency, Oxford University Press, pp. 68-119.

Halpern, L. and Körösi, G. (1995): Le pouvoir de marché: effets de taille et de monopole en Hongrie (Market power: firm size and monopoly effects in Hungary); Économie Internationale, No. 62, pp. 35-48.

Halpern, L. and Körösi, G. (1998a): Labour Market Characteristics and Profitability (Econometric Analysis of Hungarian Exporting Firms, 1986-1995); Economics of Transition, Vol. 6, No. 1, pp. 145-162.

Halpern, L. and G. Körösi (1998b): Corporate Performance in Transition (Econometric Analysis of Hungarian Exporting Firms, 1985-1994); in: Halpern, L. and C. Wyplosz (Eds.): Hungary: Towards a Market Economy; Cambridge University Press pp. 192-212.

Halpern, L. and G. Körösi (1998c): Corporate Structure and Performance in Hungary; William Davidson Institute Working Paper Series No. 187 p. 28.

Harrison, A. (1994): Productivity, Imperfect Competition and Trade Reform: Theory and Evidence', Journal of International Economics, Vol. 36, pp. 53-73.

Hay, D.A. and Liu, G.S. (1997): The Efficiency of Firms: What Difference Does Competition Make?; The Economic Journal, Vol. 107, No. 442, pp. 597-617.

Ickes, B. and Ryterman, R. (1992): Entry without Exits: Economic Selection under Socialism; mimeo, World Bank.

Kalirajan, K.P. (1997): A measure of economic efficency using returns to scale; Economics Letters, Vol. 56, No. 3, pp. 253-259. 
Konings, J. (1997): Competition and Firm Performance in Transition Economies: Evidence from Firm Level Surveys in Slovenia, Hungary and Romania; CEPR Discussion Paper No. 1770.

Konings, J. and Repkin, A. (1998): How Efficient Are Firms In Transition Countries? Firm-Level Evidence From Bulgaria and Romania; CEPR Discussion Paper No. 1839.

Major, I. (1999): The Transforming Enterprise: Company Performance After Privatization in Hungary Between 1988 and 1997; Comparative Economic Studies, Vol. 41, No. 2-3, pp. 61-110.

Nickell, S. (1996): Competition and corporate performance; Journal of Political Economy, Vol. 104, No. 4, pp. 724-746.

Nickell, S., D. Nicolitsas and Dryden, N. (1997): What Makes Firms Perform Well?; European Economic Review, Vol. 41, pp. 783-796.

Otto, G. (1999): The Solow Residual for Australia: Technology Shocks or Factor Utilization?; Economic Inquiry, Vol. 37, No. 1, pp. 136-153.

Tóth, I.J. (1999): Ownership Structure, Business Links and Performance of Firms in a Transforming Economy. The Case of Hungary; Institute of Economics, Hungarian Academy of Sciences Discussion Papers No. 1999/3.

Voszka, É. (1997): A dinoszauruszok esélyei (Prospects of Dinosaurs); Közgazdasági Szemle, Vol. 44, pp. 31-41. 


\section{Appendix A: Definitions}

All variables (except employment) were deflated, usually with four digit sectoral producer price indices. There were some - usually small-sectors, where the price index was only available at a higher level of aggregation ( 2 or 3 digit sectors). Variables are measured in million Forints at 1991 prices. The variables are:

Valued added: Sales less broadly defined material costs. Its logarithm is the dependent variable of all production functions.

Labour (L): Annual average employment at the firm.

Capital (K): Fixed assets. See data section for qualifications.

Market share: Sales of the firm divided by the market size, where market size is the sectoral production plus competing imports less exports, all measured at the four digit sectoral level. The sectoral classification of imports is based on the four-digit product classification.

Import penetration: The ratio of the sectoral imports to the above defined market size.

Concentration: The reciprocal of the number of firms in the four digit sector.

Efficiency: The error term $u$ of the frontier production function.

Profit margin: Pre-tax profits relative to sales.

Investment ratio: Change of capital value plus depreciation over the current capital value.

Large firm: A firm where the number of employees is greater than 500, or the value of fixed assets is greater than $1 \mathrm{bn} .1991$ forints or sales volume is greater than 1.5bn. 1991 forints.

Small firm: A firm where the number of employees is less than 50 , or the value of fixed assets is less than $20 \mathrm{~m} .1991$ forints or sales volume is less than $25 \mathrm{~m}$. 1991 forints.

New firm: A firm with an identifier (tax-file number) which was not in the sample in an earlier year.

Disappearing firm: A firm with an identifier (tax-file number) which was not in the sample in a later year.

Private firm: A firm where named persons (investors, employees and managers) owned more than $50 \%$ of the equity capital. Firms owned indirectly (by domestic firms) are excluded, as the parent company can be a SOE.

State owned firm: A firm where the central and local governments together owned more than $50 \%$ of the equity capital.

Foreign owned firm: Foreign investors owned more than $50 \%$ of the equity capital.

Important foreign ownership: Foreign investors owned 25-50\% of the equity capital. This category may include firms which are present at other ownership categories.

Legend to the tables: Production functions were estimated by maximum likelihood. Asterisks after the coefficients and test statistics indicate that the test is significant at 0.05 level $(*)$ or at 0.01 level $(* *)$. The null for returns to scale $(\nu)$ is that $\nu=1$. $\sigma$ denotes the standard error of the compound disturbance term $\left(\sigma^{2}=\sigma_{u}^{2}+\sigma_{v}^{2}\right)$, while $\sigma_{u} / \sigma_{v}$ stands for the ratio of the two standard errors (often denoted by $\lambda$ ). Mean inefficiency is normalized by the mean of the dependent variable. Abbreviations: Nob: number of observations; SEE: standard error of the estimation; Reset $\mathrm{y}^{2}$ : Ramsey's Reset test using the squared fitted values; Reset $\mathrm{y}^{2}, \mathrm{y}^{3}$ the same using both the squares and the cubes of the fitted values. Chow test is for structural break between the two consecutive years; for production functions it is the LR-test, while for share equations the Wald-test. All share equations were estimated by OLS using White heteroscedasticity consistent standard errors. 


\section{Appendix B: Selected estimation results}

Table 1: Single equation models: panel estimates

\begin{tabular}{|l|c|c|c|c|c|c|}
\hline & \multicolumn{3}{|c|}{ All firms } & \multicolumn{3}{c|}{ Manufacturing } \\
\hline Variable & $1990-97$ & $1990-93$ & $1994-97$ & $1990-97$ & $1990-93$ & $1994-97$ \\
\hline Constant & $-0.10 * *$ & $0.09 * *$ & $0.17 * *$ & 0.00 & $0.29 * *$ & $0.20 * *$ \\
$\mathrm{y}_{t-1}$ & $0.63 * *$ & $0.45 * *$ & $0.72 * *$ & $0.60 * *$ & $0.46 * *$ & $0.68 * *$ \\
$\log (\mathrm{L})$ & $0.28 * *$ & $0.40 * *$ & $0.22 * *$ & $0.26 * *$ & $0.31 * *$ & $0.22 * *$ \\
$\log (\mathrm{K})$ & $0.10 * *$ & $0.10 * *$ & $0.09 * *$ & $0.13 * *$ & $0.16 * *$ & $0.11 * *$ \\
import penetration & $0.02 * *$ & 0.00 & $0.02 * *$ & 0.00 & $-0.03 * *$ & $0.01 *$ \\
market share $t-1$ & $0.23 * *$ & $0.49 * *$ & $0.16 * *$ & $0.23 * *$ & $0.44 * *$ & $0.17 * *$ \\
concentration & $0.51 * *$ & $0.83 * *$ & 0.23 & $0.78 * *$ & $1.36 * *$ & $0.48 * *$ \\
Dummy for 1991 & $-0.25 * *$ & $-0.27 * *$ & & $-0.23 * *$ & $-0.24 * *$ & \\
Dummy for 1992 & $0.46 * *$ & $0.33 * *$ & & $0.59 * *$ & $0.51 * *$ & \\
Dummy for 1993 & $0.31 * *$ & $0.26 * *$ & & $0.35 * *$ & $0.32 * *$ & \\
Dummy for 1994 & $0.31 * *$ & & & $0.31 * *$ & & \\
Dummy for 1995 & $0.26 * *$ & & $-0.05 * *$ & $0.31 * *$ & & 0.00 \\
Dummy for 1996 & $0.27 * *$ & & $-0.05 * *$ & $0.31 * *$ & & -0.01 \\
Dummy for 1997 & $0.28 * *$ & & $-0.03 * *$ & $0.31 * *$ & & 0.00 \\
$\sigma$ & $0.78 * *$ & $0.90 * *$ & $0.70 * *$ & $0.75 * *$ & $0.91 * *$ & $0.66 * *$ \\
$\sigma_{u} / \sigma_{v}$ & $1.40 * *$ & $1.49 * *$ & $1.35 * *$ & $1.58 * *$ & $1.93 * *$ & $1.38 * *$ \\
\hline short-run ret. to scale & $0.38 * *$ & $0.50 * *$ & $0.30 * *$ & $0.40 * *$ & $0.47 * *$ & $0.34 * *$ \\
long-run ret. to scale & $1.02 * *$ & $0.92 * *$ & $1.08 * *$ & 0.99 & $0.88 * *$ & $1.05 * *$ \\
\hline Nob & 45777 & 13345 & 32432 & 17292 & 5320 & 11972 \\
Mean of dep.var & 3.38 & 3.64 & 3.27 & 3.63 & 3.75 & 3.58 \\
S.dev of dep.var & 1.47 & 1.36 & 1.49 & 1.54 & 1.43 & 1.59 \\
$\mathrm{R}^{2}$ & 0.83 & 0.75 & 0.87 & 0.86 & 0.79 & 0.90 \\
Mean inefficiency $(\%)$ & -14.63 & -16.07 & -13.46 & -13.65 & -16.75 & -11.66 \\
\hline
\end{tabular}


Table 2: Single equation models: bi-annual panels, all firms

\begin{tabular}{|c|c|c|c|c|c|c|c|}
\hline Variable & $1990-1$ & $1991-2$ & $1992-3$ & $1993-4$ & $1994-5$ & $1995-6$ & $1996-7$ \\
\hline Constant & $0.45 * *$ & $-0.28 * *$ & $0.22 * *$ & $0.23 * *$ & $0.18 * *$ & $0.12 * *$ & $0.13 * *$ \\
\hline $\mathrm{y}_{t-1}$ & $0.67 * *$ & $0.30 * *$ & $0.39 * *$ & $0.60 * *$ & $0.69 * *$ & $0.72 * *$ & $0.74 * *$ \\
\hline $\log (\mathrm{L})$ & $0.17 * *$ & $0.54 * *$ & $0.48 * *$ & $0.31 * *$ & $0.24 * *$ & $0.22 * *$ & $0.20 * *$ \\
\hline $\log (K)$ & $0.10 * *$ & $0.11 * *$ & $0.10 * *$ & $0.08 * *$ & $0.09 * *$ & $0.09 * *$ & $0.08 * *$ \\
\hline import penetration & -0.07 & 0.02 & 0.00 & 0.00 & $0.03 * *$ & $0.04 * *$ & $0.01 *$ \\
\hline market share ${ }_{t-1}$ & -0.13 & $0.76 * *$ & $0.79 * *$ & $0.29 * *$ & $0.23 * *$ & $0.21 * *$ & $0.13 * *$ \\
\hline concentration & $1.78 * *$ & $0.93 *$ & 0.33 & 0.37 & -0.01 & 0.26 & $0.41 *$ \\
\hline year $_{2}$ & $-0.23 * *$ & $0.53 * *$ & $-0.03 *$ & 0.01 & $-0.05 * *$ & 0.01 & $0.02 * *$ \\
\hline$\sigma$ & $0.89 * *$ & $0.92 * *$ & $0.88 * *$ & $0.78 * *$ & $0.69 * *$ & $0.71 * *$ & $0.70 * *$ \\
\hline$\sigma_{u} / \sigma_{v}$ & $1.96 * *$ & $1.64 * *$ & $1.37 * *$ & $1.37 * *$ & $1.35 * *$ & $1.33 * *$ & $1.36 * *$ \\
\hline short-run ret. to scale & $0.27 * *$ & $0.65 * *$ & $0.58 * *$ & $0.39 * *$ & $0.33 * *$ & $0.31 * *$ & $0.29 * *$ \\
\hline long-run ret. t & $0.81 * *$ & $0.93 * *$ & $0.95 * *$ & 0.99 & $1.05 * *$ & $1.07 * *$ & $1.10 * *$ \\
\hline Nob & 3552 & 4870 & 9793 & 12040 & 13814 & 16881 & 18618 \\
\hline Mean of dep.var & 4.08 & 3.68 & 3.49 & 3.52 & 3.46 & 3.27 & 3.12 \\
\hline S.dev of dep.var & 1.33 & 1.31 & 1.34 & 1.37 & 1.43 & 1.48 & 1.52 \\
\hline $\mathrm{R}^{2}$ & 0.76 & 0.73 & 0.74 & 0.81 & 0.86 & 0.86 & 0.87 \\
\hline Mean inefficiency (\%) & -15.05 & -16.84 & -16.09 & -13.99 & -12.59 & -13.59 & -14.18 \\
\hline Chow test $\left(\chi^{2}\right)$ & $22.19 * *$ & $353.28 * *$ & $518.09 * *$ & $149.54 * *$ & $28.13 * *$ & $36.17 * *$ & 5.89 \\
\hline
\end{tabular}

Table 3: Single equation models: bi-annual panels, manufacturing

\begin{tabular}{|l|c|c|c|c|c|c|c|}
\hline Variable & $1990-1$ & $1991-2$ & $1992-3$ & $1993-4$ & $1994-5$ & $1995-6$ & $1996-7$ \\
\hline Constant & $0.47 * *$ & $-0.24 * *$ & $0.60 * *$ & $0.49 * *$ & $0.28 * *$ & $0.16 * *$ & $0.16 * *$ \\
y $t-1$ & $0.64 * *$ & $0.29 * *$ & $0.39 * *$ & $0.55 * *$ & $0.64 * *$ & $0.67 * *$ & $0.71 * *$ \\
$\log (\mathrm{L})$ & $0.17 * *$ & $0.51 * *$ & $0.40 * *$ & $0.27 * *$ & $0.23 * *$ & $0.23 * *$ & $0.21 * *$ \\
$\log (\mathrm{K})$ & $0.13 * *$ & $0.16 * *$ & $0.16 * *$ & $0.15 * *$ & $0.13 * *$ & $0.12 * *$ & $0.11 * *$ \\
import penetration & 1.07 & -0.03 & $-0.03 * *$ & $-0.02 * *$ & $0.01 *$ & $0.02 * *$ & 0.01 \\
market share $t-1$ & $-0.29 *$ & $0.60 * *$ & $0.87 * *$ & $0.43 * *$ & $0.41 * *$ & $0.29 * *$ & $0.13 * *$ \\
concentration & $2.88 * *$ & $1.25 *$ & 0.74 & $0.93 *$ & 0.16 & $0.50 *$ \\
year & $-0.19 * *$ & $0.68 * *$ & $-0.13 * *$ & -0.03 & 0.00 & -0.01 & $0.60 * *$ \\
$\sigma$ & $0.93 * *$ & $0.96 * *$ & $0.88 * *$ & $0.78 * *$ & $0.67 * *$ & $0.65 * *$ & $0.66 * *$ \\
$\sigma_{u} / \sigma_{v}$ & $2.24 * *$ & $2.30 * *$ & $1.85 * *$ & $1.77 * *$ & $1.33 * *$ & $1.17 * *$ & $1.44 * *$ \\
\hline short-run ret. to scale & $0.30 * *$ & $0.67 * *$ & $0.56 * *$ & $0.41 * *$ & $0.35 * *$ & $0.34 * *$ & $0.32 * *$ \\
long-run ret. to scale & $0.83 * *$ & $0.94 * *$ & $0.91 * *$ & $0.92 * *$ & 0.97 & $1.04 *$ & $1.09 * *$ \\
\hline Nob & 1850 & 1894 & 3470 & 4355 & 5102 & 6232 & 6870 \\
Mean of dep.var & 3.94 & 3.75 & 3.66 & 3.76 & 3.74 & 3.58 & 3.46 \\
S.dev of dep.var & 1.37 & 1.42 & 1.45 & 1.48 & 1.53 & 1.57 \\
$\mathrm{R}^{2}$ & 0.76 & 0.78 & 0.81 & 0.85 & 0.88 & 0.89 \\
Mean inefficiency $(\%)$ & -16.61 & -18.33 & -16.54 & -13.99 & -11.17 & -10.85 \\
Chow test $\left(\chi^{2}\right)$ & $54.48 * *$ & $92.90 * *$ & $165.52 * *$ & $48.61 * *$ & $13.91 *$ & $22.44 * *$ & $16.25 * *$ \\
\hline
\end{tabular}


Table 4: Two-equation models: panel estimates

\begin{tabular}{|c|c|c|c|c|c|c|}
\hline & \multicolumn{3}{|c|}{ All firms } & \multicolumn{3}{|c|}{ Manufacturing } \\
\hline Variable & 1990-97 & 1990-93 & $1994-97$ & $1990-97$ & 1990-93 & 1994-97 \\
\hline \multicolumn{7}{|l|}{ Production function } \\
\hline Constant & $-0.13 * *$ & 0.02 & $0.16 * *$ & -0.04 & $0.21 * *$ & $0.19 * *$ \\
\hline $\mathrm{y}_{t-1}$ & $0.63 * *$ & $0.46 * *$ & $0.72 * *$ & $0.60 * *$ & $0.47 * *$ & $0.69 * *$ \\
\hline $\log (\mathrm{L})$ & $0.29 * *$ & $0.41 * *$ & $0.22 * *$ & $0.27 * *$ & $0.32 * *$ & $0.22 * *$ \\
\hline $\log (K)$ & $0.10 * *$ & $0.10 * *$ & $0.09 * *$ & $0.14 * *$ & $0.16 * *$ & $0.12 * *$ \\
\hline Dummy for 1991 & $-0.25 * *$ & $-0.27 * *$ & & $-0.24 * *$ & $-0.25 * *$ & \\
\hline Dummy for 1992 & $0.46 * *$ & $0.34 * *$ & & $0.60 * *$ & $0.51 * *$ & \\
\hline Dummy for 1993 & $0.31 * *$ & $0.27 * *$ & & $0.35 * *$ & $0.32 * *$ & \\
\hline Dummy for 1994 & $0.32 * *$ & & & $0.32 * *$ & & \\
\hline Dummy for 1995 & $0.27 * *$ & & $-0.05 * *$ & $0.32 * *$ & & 0.00 \\
\hline Dummy for 1996 & $0.28 * *$ & & $-0.05 * *$ & $0.31 * *$ & & -0.01 \\
\hline Dummy for 1997 & $0.30 * *$ & & $-0.02 *$ & $0.32 * *$ & & 0.00 \\
\hline$\sigma$ & $0.77 * *$ & $0.90 * *$ & $0.70 * *$ & $0.75 * *$ & $0.91 * *$ & $0.66 * *$ \\
\hline$\sigma_{u} / \sigma_{v}$ & $1.39 * *$ & $1.45 * *$ & $1.35 * *$ & $1.54 * *$ & $1.86 * *$ & $1.35 * *$ \\
\hline short-run ret. to scale & $0.39 * *$ & $0.51 * *$ & $0.31 * *$ & $0.40 * *$ & $0.49 * *$ & $0.34 * *$ \\
\hline long-run ret. to scale & $1.05 * *$ & $0.95 * *$ & $1.10 * *$ & 1.02 & $0.92 * *$ & $1.07 * *$ \\
\hline Nob & 45777 & 13345 & 32432 & 17292 & 5320 & 11972 \\
\hline Mean of dep.var & 3.38 & 3.64 & 3.27 & 3.63 & 3.75 & 3.58 \\
\hline S.dev of dep.var & 1.47 & 1.36 & 1.49 & 1.54 & 1.43 & 1.59 \\
\hline $\mathrm{R}^{2}$ & 0.83 & 0.75 & 0.87 & 0.86 & 0.79 & 0.90 \\
\hline Mean inefficiency (\%) & -14.57 & -15.88 & -13.43 & -13.52 & -16.53 & -11.57 \\
\hline \multicolumn{7}{|l|}{ Market share equation } \\
\hline Constant & $0.01 * *$ & $0.01 * *$ & $0.01 * *$ & $0.02 * *$ & $0.01 * *$ & $0.02 * *$ \\
\hline market share $_{t-1}$ & $0.63 * *$ & $0.81 * *$ & $0.59 * *$ & $0.50 * *$ & $0.82 * *$ & $0.44 * *$ \\
\hline efficiency & $1.18 * *$ & $0.82 * *$ & $1.42 * *$ & $1.52 * *$ & $0.88 * *$ & $2.17 * *$ \\
\hline import penetration & $-0.01 * *$ & $-0.01 * *$ & $-0.01 * *$ & $-0.01 * *$ & $-0.01 * *$ & $-0.01 * *$ \\
\hline concentration & $0.73 * *$ & $0.61 * *$ & $0.70 * *$ & $0.77 * *$ & $0.45 * *$ & $0.77 * *$ \\
\hline Mean of dep.var & 0.02 & 0.03 & 0.02 & 0.03 & 0.03 & 0.03 \\
\hline S.dev of dep.var & 0.10 & 0.10 & 0.10 & 0.10 & 0.10 & 0.10 \\
\hline SEE & 0.06 & 0.05 & 0.06 & 0.07 & 0.04 & 0.07 \\
\hline $\mathrm{R}^{2}$ & 0.69 & 0.77 & 0.66 & 0.57 & 0.80 & 0.52 \\
\hline White-hetero & $28072 * *$ & $397 * *$ & $20513 * *$ & $16488 * *$ & $2895 * *$ & $10882 * *$ \\
\hline Reset $\mathrm{y}^{2}, \mathrm{y}^{3}$ & $13924 * *$ & $12 * *$ & $16764 * *$ & $14316 * *$ & $7 * *$ & $14017 * *$ \\
\hline \multicolumn{7}{|l|}{ Corr of rel. efficiency } \\
\hline $\begin{array}{l}\text { profit margin } \\
\text { investment rate }\end{array}$ & 0.17 & 0.17 & 0.17 & 0.40 & 0.37 & 0.42 \\
\hline
\end{tabular}


Table 5: Two-equation models: bi-annual panels, all firms

\begin{tabular}{|c|c|c|c|c|c|c|c|}
\hline Variable & $1990-1$ & $1991-2$ & $1992-3$ & $1993-4$ & $1994-5$ & $1995-6$ & $1996-7$ \\
\hline \multicolumn{8}{|l|}{ Production function } \\
\hline Constant & $0.44 * *$ & $-0.41 * *$ & $0.13 * *$ & $0.19 * *$ & $0.15 * *$ & $0.10 * *$ & $0.11 * *$ \\
\hline $\mathrm{y}_{t-1}$ & $0.67 * *$ & $0.30 * *$ & $0.40 * *$ & $0.61 * *$ & $0.69 * *$ & $0.72 * *$ & $0.74 * *$ \\
\hline $\log (\mathrm{L})$ & $0.17 * *$ & $0.56 * *$ & $0.49 * *$ & $0.32 * *$ & $0.24 * *$ & $0.22 * *$ & $0.21 * *$ \\
\hline $\log (K)$ & $0.11 * *$ & $0.11 * *$ & $0.10 * *$ & $0.08 * *$ & $0.09 * *$ & $0.09 * *$ & $0.08 * *$ \\
\hline year $_{2}$ & $-0.24 * *$ & $0.54 * *$ & -0.02 & 0.01 & $-0.05 * *$ & 0.01 & $0.02 * *$ \\
\hline$\sigma$ & $0.90 * *$ & $0.92 * *$ & $0.88 * *$ & $0.78 * *$ & $0.69 * *$ & $0.71 * *$ & $0.70 * *$ \\
\hline$\sigma_{u} / \sigma_{v}$ & $1.94 * *$ & $1.55 * *$ & $1.33 * *$ & $1.36 * *$ & $1.34 * *$ & $1.32 * *$ & $1.35 * *$ \\
\hline short-run ret. to scale & $0.27 * *$ & $0.67 * *$ & $0.59 * *$ & $0.40 * *$ & $0.33 * *$ & $0.31 * *$ & $0.29 * *$ \\
\hline long-run ret. to scale & $0.83 * *$ & $0.97 *$ & 0.99 & 1.01 & $1.08 * *$ & $1.11 * *$ & $1.12 * *$ \\
\hline Nob & 3552 & 4870 & 9793 & 12040 & 13814 & 16881 & 18618 \\
\hline Mean of dep.var & 4.08 & 3.68 & 3.49 & 3.52 & 3.46 & 3.27 & 3.12 \\
\hline S.dev of dep.var & 1.33 & 1.31 & 1.34 & 1.37 & 1.43 & 1.48 & 1.52 \\
\hline $\mathrm{R}^{2}$ & 0.76 & 0.73 & 0.74 & 0.81 & 0.86 & 0.86 & 0.87 \\
\hline Mean inefficiency (\%) & -15.05 & -16.44 & -15.86 & -13.92 & -12.54 & -13.57 & -14.15 \\
\hline Chow test $\left(\chi^{2}\right)$ & $15.39 * *$ & $289.37 * *$ & $500.50 * *$ & $140.34 * *$ & $15.59 * *$ & $31.97 * *$ & 3.15 \\
\hline \multicolumn{8}{|l|}{ Market share equation } \\
\hline Constant & 0.00 & $0.01 * *$ & $0.01 * *$ & $0.01 * *$ & $0.01 * *$ & $0.01 * *$ & $0.01 * *$ \\
\hline market share $t_{-1}$ & $0.92 * *$ & $0.72 * *$ & $0.75 * *$ & $0.81 * *$ & $0.87 * *$ & $0.87 * *$ & $0.48 * *$ \\
\hline efficiency & $0.87 * *$ & $1.03 * *$ & $1.05 * *$ & $1.25 * *$ & $1.46 * *$ & $1.17 * *$ & $1.35 * *$ \\
\hline import penetration & 0.00 & $-0.02 * *$ & $-0.01 * *$ & $0.00 *$ & $0.00 *$ & $0.00 * *$ & $-0.01 * *$ \\
\hline concentration & $0.15 *$ & $0.94 * *$ & $0.94 * *$ & $0.46 *$ & 0.07 & 0.21 & $0.93 * *$ \\
\hline year $_{2}$ & $0.00 * *$ & $0.01 * *$ & $0.00 * *$ & 0.00 & $0.00 * *$ & 0.00 & 0.00 \\
\hline Mean of dep.var & 0.03 & 0.03 & 0.02 & 0.02 & 0.02 & 0.02 & 0.02 \\
\hline S.dev of dep.var & 0.11 & 0.09 & 0.10 & 0.11 & 0.11 & 0.10 & 0.09 \\
\hline SEE & 0.03 & 0.05 & 0.05 & 0.05 & 0.05 & 0.05 & 0.06 \\
\hline $\mathrm{R}^{2}$ & 0.92 & 0.66 & 0.72 & 0.81 & 0.80 & 0.80 & 0.62 \\
\hline White-hetero & $1503 * *$ & $1949 * *$ & $690 * *$ & $942 * *$ & $379 * *$ & $543 * *$ & $17333 * *$ \\
\hline Reset $y^{2}$ & $285.52 * *$ & $64.91 * *$ & $5.96 *$ & $38.91 * *$ & $385.89 * *$ & $405.29 * *$ & $29036.10 * *$ \\
\hline Reset $\mathrm{y}^{2}, \mathrm{y}^{3}$ & $187.73 * *$ & $38.96 * *$ & $12.39 * *$ & $142.93 * *$ & $266.72 * *$ & $298.46 * *$ & $18400.48 * *$ \\
\hline Chow test (F) & 6.48 & $21.76 * *$ & $17.90 * *$ & $9.55 *$ & 7.38 & $9.80 *$ & 8.88 \\
\hline
\end{tabular}


Table 6: Two-equation models: bi-annual panels, manufacturing

\begin{tabular}{|c|c|c|c|c|c|c|c|}
\hline Variable & $1990-1$ & $1991-2$ & $1992-3$ & $1993-4$ & $1994-5$ & $1995-6$ & $1996-7$ \\
\hline \multicolumn{8}{|l|}{ Production function } \\
\hline Constant & $0.51 * *$ & $-0.36 * *$ & $0.48 * *$ & $0.43 * *$ & $0.24 * *$ & $0.14 * *$ & $0.14 * *$ \\
\hline $\mathrm{y}_{t-1}$ & $0.64 * *$ & $0.30 * *$ & $0.40 * *$ & $0.56 * *$ & $0.65 * *$ & $0.68 * *$ & $0.71 * *$ \\
\hline $\log (\mathrm{L})$ & $0.16 * *$ & $0.53 * *$ & $0.41 * *$ & $0.27 * *$ & $0.23 * *$ & $0.23 * *$ & $0.21 * *$ \\
\hline $\log (K)$ & $0.14 * *$ & $0.16 * *$ & $0.17 * *$ & $0.15 * *$ & $0.13 * *$ & $0.12 * *$ & $0.11 * *$ \\
\hline year $_{2}$ & $-0.21 * *$ & $0.70 * *$ & $-0.13 * *$ & -0.03 & 0.00 & -0.01 & 0.01 \\
\hline$\sigma$ & $0.93 * *$ & $0.95 * *$ & $0.87 * *$ & $0.78 * *$ & $0.67 * *$ & $0.65 * *$ & $0.65 * *$ \\
\hline$\sigma_{u} / \sigma_{v}$ & $2.18 * *$ & $2.15 * *$ & $1.77 * *$ & $1.73 * *$ & $1.30 * *$ & $1.15 * *$ & $1.42 * *$ \\
\hline short-run ret. to scale & $0.30 * *$ & $0.69 * *$ & $0.58 * *$ & $0.42 * *$ & $0.36 * *$ & $0.35 * *$ & $0.32 * *$ \\
\hline long-run ret. to scale & $0.83 * *$ & 0.98 & $0.96 *$ & $0.96 *$ & 1.01 & $1.07 * *$ & $1.11 * *$ \\
\hline Nob & 1850 & 1894 & 3470 & 4355 & 5102 & 6232 & 6870 \\
\hline Mean of dep.var & 3.94 & 3.75 & 3.66 & 3.76 & 3.74 & 3.58 & 3.46 \\
\hline S.dev of dep.var & 1.37 & 1.42 & 1.45 & 1.48 & 1.53 & 1.57 & 1.62 \\
\hline $\mathrm{R}^{2}$ & 0.76 & 0.78 & 0.81 & 0.85 & 0.88 & 0.89 & 0.90 \\
\hline Mean inefficiency (\%) & -16.56 & -17.96 & -16.31 & -13.88 & -11.04 & -10.75 & -12.04 \\
\hline Chow test $\left(\chi^{2}\right)$ & $38.65 * *$ & $60.12 * *$ & $166.16 * *$ & $47.76 * *$ & $6.67 *$ & $16.92 * *$ & 5.28 \\
\hline \multicolumn{8}{|l|}{ Market share equation } \\
\hline Constant & 0.00 & $0.01 *$ & $0.02 * *$ & $0.01 * *$ & $0.01 * *$ & $0.01 * *$ & $0.02 * *$ \\
\hline market share $e_{t-1}$ & $0.95 * *$ & $0.67 * *$ & $0.75 * *$ & $0.98 * *$ & $1.01 * *$ & $0.93 * *$ & $0.32 * *$ \\
\hline efficiency & 0.83 & $0.87 *$ & $1.28 * *$ & $1.33 * *$ & $1.68 * *$ & $1.76 * *$ & $1.99 * *$ \\
\hline import penetration & 0.01 & $-0.02 * *$ & $-0.01 * *$ & $0.00 *$ & 0.00 & $0.00 * *$ & $-0.01 * *$ \\
\hline concentration & 0.02 & $1.07 * *$ & $0.69 * *$ & 0.03 & -0.03 & $0.21 *$ & $0.93 * *$ \\
\hline year $_{2}$ & $0.00 *$ & 0.01 & $-0.01 *$ & 0.00 & $0.00 * *$ & 0.00 & 0.00 \\
\hline Mean of dep.var & 0.04 & 0.03 & 0.03 & 0.03 & 0.03 & 0.03 & 0.03 \\
\hline S.dev of dep.var & 0.11 & 0.10 & 0.09 & 0.10 & 0.10 & 0.10 & 0.10 \\
\hline SEE & 0.03 & 0.06 & 0.05 & 0.03 & 0.03 & 0.04 & 0.07 \\
\hline $\mathrm{R}^{2}$ & 0.91 & 0.65 & 0.73 & 0.89 & 0.90 & 0.85 & 0.46 \\
\hline White-hetero & $798 * *$ & $1267 * *$ & $2285 * *$ & $735 * *$ & $974 * *$ & $599 * *$ & $5013 * *$ \\
\hline Reset $\mathrm{y}^{2}$ & $230.25 * *$ & $11.09 * *$ & $14.78 * *$ & $384.67 * *$ & $228.61 * *$ & 0.70 & $15634.67 * *$ \\
\hline Reset $\mathrm{y}^{2}, \mathrm{y}^{3}$ & $152.44 * *$ & $50.82 * *$ & $7.53 * *$ & $205.92 * *$ & $129.49 * *$ & $76.23 * *$ & $7818.86 * *$ \\
\hline Chow test $(\mathrm{F})$ & 7.80 & $27.63 * *$ & $59.32 * *$ & 2.15 & 3.25 & $17.61 * *$ & $46.59 * *$ \\
\hline
\end{tabular}


Table 7-A: Single equation models: all firms

\begin{tabular}{|c|c|c|c|c|c|c|c|c|}
\hline Variable & 1990 & 1991 & 1992 & 1993 & 1994 & 1995 & 1996 & 1997 \\
\hline Constant & $0.55 * *$ & 0.04 & 0.10 & $0.28 * *$ & $0.21 * *$ & $0.11 * *$ & $0.14 * *$ & $0.14 * *$ \\
\hline $\mathrm{y}_{t-1}$ & $0.68 * *$ & $0.63 * *$ & $0.23 * *$ & $0.54 * *$ & $0.69 * *$ & $0.69 * *$ & $0.74 * *$ & $0.73 * *$ \\
\hline $\log (\mathrm{L})$ & $0.13 * *$ & $0.24 * *$ & $0.62 * *$ & $0.35 * *$ & $0.24 * *$ & $0.24 * *$ & $0.20 * *$ & $0.21 * *$ \\
\hline $\log (\mathrm{K})$ & $0.10 * *$ & $0.10 * *$ & $0.10 * *$ & $0.08 * *$ & $0.08 * *$ & $0.09 * *$ & $0.08 * *$ & $0.09 * *$ \\
\hline import penetration & 0.54 & -0.08 & 0.00 & -0.01 & 0.01 & $0.05 * *$ & $0.02 *$ & 0.01 \\
\hline market share $_{t-1}$ & 0.03 & $-0.34 * *$ & $1.65 * *$ & $0.38 * *$ & $0.23 * *$ & $0.25 * *$ & $0.18 *$ & $0.12 * *$ \\
\hline concentration & $1.66 * *$ & 1.62 & 0.64 & 0.71 & -0.21 & 0.08 & 0.44 & 0.33 \\
\hline$\sigma$ & $0.85 * *$ & $0.97 * *$ & $0.87 * *$ & $0.86 * *$ & $0.67 * *$ & $0.71 * *$ & $0.71 * *$ & $0.70 * *$ \\
\hline$\sigma_{u} / \sigma_{v}$ & $1.85 * *$ & $2.22 * *$ & $1.50 * *$ & $1.34 * *$ & $1.49 * *$ & $1.28 * *$ & $1.39 * *$ & $1.33 * *$ \\
\hline short-run ret. to scale & $0.23 * *$ & $0.34 * *$ & $0.72 * *$ & $0.44 * *$ & $0.32 * *$ & $0.33 * *$ & $0.28 * *$ & $0.30 * *$ \\
\hline long-run ret. to scale & $0.73 * *$ & 0.92 & $0.93 * *$ & $0.95 * *$ & 1.02 & $1.06 * *$ & $1.09 * *$ & $1.10 * *$ \\
\hline Nob & 2156 & 1396 & 3474 & 6319 & 5721 & 8093 & 8788 & 9830 \\
\hline Mean of dep.var & 4.22 & 3.85 & 3.62 & 3.42 & 3.64 & 3.34 & 3.20 & 3.06 \\
\hline S.dev of dep.var & 1.31 & 1.34 & 1.29 & 1.35 & 1.38 & 1.45 & 1.50 & 1.54 \\
\hline $\mathrm{R}^{2}$ & 0.77 & 0.74 & 0.75 & 0.76 & 0.86 & 0.85 & 0.86 & 0.87 \\
\hline Mean inefficiency (\%) & -13.55 & -17.71 & -15.72 & -15.86 & -11.97 & -13.06 & -14.10 & -14.24 \\
\hline
\end{tabular}

Table 7-B: Mean relative inefficiency for subsets of observations

\begin{tabular}{|l|r|r|c|c|c|c|c|c|}
\hline Variable & 1990 & 1991 & 1992 & 1993 & 1994 & 1995 & 1996 & 1997 \\
\hline Manufacturing & -13.6 & -17.6 & -14.7 & -15.1 & -11.7 & -12.5 & -13.4 & -13.6 \\
\hline Engineering & -13.7 & -20.1 & -14.3 & -14.4 & -11.4 & -11.5 & -12.4 & -12.6 \\
Chemical ind. & -12.6 & -15.5 & -11.9 & -12.7 & -9.9 & -13.0 & -12.7 & -13.4 \\
Pharmaceutical ind. & -9.0 & -10.9 & -10.6 & -14.1 & -8.7 & -13.6 & -15.0 & -12.7 \\
Food ind. & -11.9 & -14.5 & -12.6 & -15.5 & -13.4 & -12.9 & -15.4 & -15.5 \\
Light ind. & -13.7 & -17.5 & -16.3 & -16.2 & -11.9 & -13.3 & -13.7 & -14.0 \\
Other ind. & -14.9 & -15.9 & -14.3 & -13.8 & -10.6 & -12.0 & -13.3 & -13.3 \\
\hline Agriculture & & & -17.2 & -18.9 & -12.3 & -13.4 & -16.2 & -16.9 \\
Construction & -12.7 & -17.2 & -15.6 & -15.5 & -11.7 & -14.3 & -14.2 & -13.7 \\
Trade & -13.7 & -17.9 & -16.0 & -15.6 & -12.5 & -13.4 & -14.0 & -14.2 \\
Services & -17.6 & -20.2 & -13.9 & -14.1 & -11.0 & -11.6 & -13.1 & -12.8 \\
\hline Owner: Private & & & -16.4 & -14.3 & -11.6 & -12.5 & -14.2 & -13.9 \\
$\quad$ Government & -13.3 & -18.2 & -15.7 & -16.5 & -12.7 & -14.1 & -14.7 & -14.8 \\
$\quad$ Foreign & -9.2 & -17.9 & -9.7 & -12.3 & -9.0 & -11.2 & -11.9 & -11.9 \\
$\quad$ Imp. foreign & -7.5 & -20.7 & -12.6 & -13.1 & -11.0 & -11.4 & -12.9 & -13.0 \\
$\quad$ Other & -13.7 & -17.3 & -16.3 & -16.8 & -12.5 & -13.5 & -14.5 & -14.9 \\
\hline Size: Small & -13.8 & -17.0 & -15.5 & -15.8 & -12.2 & -13.2 & -14.2 & -14.3 \\
$\quad$ Medium & -13.8 & -18.2 & -16.6 & -16.4 & -11.9 & -13.0 & -14.1 & -14.3 \\
Large & -13.0 & -17.6 & -12.7 & -13.8 & -10.9 & -11.9 & -12.6 & -12.8 \\
\hline
\end{tabular}


Table 8-A: Two-equation models: all firms

\begin{tabular}{|c|c|c|c|c|c|c|c|c|}
\hline Variable & 1990 & 1991 & 1992 & 1993 & 1994 & 1995 & 1996 & 1997 \\
\hline \multicolumn{9}{|l|}{ Production function } \\
\hline Constant & $0.51 * *$ & 0.08 & -0.10 & $0.24 * *$ & $0.18 * *$ & $0.09 * *$ & $0.13 * *$ & $0.13 * *$ \\
\hline $\mathrm{y}_{t-1}$ & $0.69 * *$ & $0.62 * *$ & $0.24 * *$ & $0.55 * *$ & $0.69 * *$ & $0.69 * *$ & $0.75 * *$ & $0.73 * *$ \\
\hline $\log (\mathrm{L})$ & $0.13 * *$ & $0.24 * *$ & $0.65 * *$ & $0.36 * *$ & $0.25 * *$ & $0.24 * *$ & $0.20 * *$ & $0.21 * *$ \\
\hline $\log (\mathrm{K})$ & $0.11 * *$ & $0.10 * *$ & $0.10 * *$ & $0.08 * *$ & $0.08 * *$ & $0.10 * *$ & $0.08 * *$ & $0.09 * *$ \\
\hline$\sigma$ & $0.84 * *$ & $0.98 * *$ & $0.85 * *$ & $0.86 * *$ & $0.67 * *$ & $0.71 * *$ & $0.71 * *$ & $0.70 * *$ \\
\hline$\sigma_{u} / \sigma_{v}$ & $1.80 * *$ & $2.27 * *$ & $1.35 * *$ & $1.33 * *$ & $1.48 * *$ & $1.26 * *$ & $1.38 * *$ & $1.32 * *$ \\
\hline short-run ret. to scale & $0.24 * *$ & $0.34 * *$ & $0.75 * *$ & $0.44 * *$ & $0.32 * *$ & $0.34 * *$ & $0.28 * *$ & $0.30 * *$ \\
\hline long-run ret. to scale & $0.77 * *$ & $0.91 *$ & 0.99 & 0.98 & $1.05 *$ & $1.09 * *$ & $1.12 * *$ & $1.12 * *$ \\
\hline Nob & 2156 & 1396 & 3474 & 6319 & 5721 & 8093 & 8788 & 9830 \\
\hline Mean of dep.var & 4.22 & 3.85 & 3.62 & 3.42 & 3.64 & 3.34 & 3.20 & 3.06 \\
\hline S.dev of dep.var & 1.31 & 1.34 & 1.29 & 1.35 & 1.38 & 1.45 & 1.50 & 1.54 \\
\hline $\mathrm{R}^{2}$ & 0.77 & 0.73 & 0.74 & 0.76 & 0.86 & 0.85 & 0.86 & 0.87 \\
\hline Mean inefficiency (\%) & -13.46 & -17.91 & -14.97 & -15.81 & -11.91 & -13.00 & -14.10 & -14.18 \\
\hline \multicolumn{9}{|l|}{ Market share equation } \\
\hline Constant & 0.00 & 0.01 & $0.02 * *$ & $0.00 * *$ & $0.01 * *$ & $0.01 * *$ & $0.01 * *$ & $0.01 * *$ \\
\hline market $\operatorname{share}_{t-1}$ & $0.89 * *$ & $0.97 * *$ & $0.54 * *$ & $0.83 * *$ & $0.79 * *$ & $0.97 * *$ & $0.81 * *$ & $0.36 * *$ \\
\hline efficiency & $0.50 * *$ & $1.30 *$ & $1.75 * *$ & $0.86 * *$ & $1.65 * *$ & $1.26 * *$ & $1.09 * *$ & $1.32 * *$ \\
\hline import penetration & -0.02 & 0.00 & $-0.02 * *$ & -0.01 & $0.00 * *$ & 0.00 & $0.00 * *$ & $-0.01 * *$ \\
\hline concentration & $0.17 *$ & 0.15 & $1.27 * *$ & 0.68 & 0.21 & -0.07 & $0.43 *$ & $1.10 * *$ \\
\hline Mean of dep.var & 0.03 & 0.03 & 0.02 & 0.02 & 0.02 & 0.02 & 0.02 & 0.02 \\
\hline S.dev of dep.var & 0.10 & 0.11 & 0.08 & 0.11 & 0.11 & 0.11 & 0.09 & 0.09 \\
\hline SEE & 0.03 & 0.04 & 0.05 & 0.05 & 0.04 & 0.05 & 0.04 & 0.06 \\
\hline $\mathrm{R}^{2}$ & 0.94 & 0.89 & 0.55 & 0.78 & 0.87 & 0.76 & 0.85 & 0.54 \\
\hline White-hetero & $343 * *$ & $879 * *$ & $950 * *$ & $465 * *$ & $3689 * *$ & $281 * *$ & $3344 * *$ & $8575 * *$ \\
\hline Reset $y^{2}$ & $114.12 * *$ & $186.79 * *$ & $109.63 * *$ & 0.35 & $227.61 * *$ & $89.57 * *$ & $225.25 * *$ & $22005.68 * *$ \\
\hline Reset $\mathrm{y}^{2}, \mathrm{y}^{3}$ & $57.62 * *$ & $173.21 * *$ & $54.84 * *$ & 0.57 & $322.26 * *$ & $55.98 * *$ & $381.02 * *$ & $14929.58 * *$ \\
\hline \multicolumn{9}{|l|}{ Corr of rel. efficiency } \\
\hline $\begin{array}{l}\text { profit margin } \\
\text { investment rate }\end{array}$ & 0.32 & 0.48 & 0.33 & 0.18 & 0.24 & 0.16 & 0.17 & \\
\hline
\end{tabular}

Table 8-B: Mean relative inefficiency for subsets of observations

\begin{tabular}{|l|r|r|r|r|r|r|r|r|}
\hline Variable & 1990 & 1991 & 1992 & 1993 & 1994 & 1995 & 1996 & 1997 \\
\hline Manufacturing & -13.4 & -17.8 & -13.9 & -15.0 & -11.6 & -12.3 & -13.3 & -13.5 \\
\hline Engineering & -13.6 & -20.4 & -13.6 & -14.4 & -11.2 & -11.2 & -12.2 & -12.4 \\
Chemical ind. & -12.5 & -16.0 & -10.7 & -12.7 & -9.9 & -12.6 & -12.5 & -13.3 \\
Pharmaceutical ind. & -9.0 & -11.7 & -9.6 & -14.1 & -8.7 & -13.5 & -15.0 & -12.7 \\
Food ind. & -11.8 & -14.5 & -12.4 & -15.5 & -13.4 & -12.9 & -15.5 & -15.5 \\
Light ind. & -13.6 & -17.7 & -15.4 & -16.2 & -11.8 & -13.0 & -13.6 & -13.9 \\
Other ind. & -14.1 & -16.0 & -13.0 & -13.6 & -10.6 & -11.9 & -13.2 & -13.2 \\
\hline Agriculture & & & -16.7 & -19.0 & -12.3 & -13.5 & -16.2 & -16.9 \\
Construction & -12.7 & -17.4 & -14.8 & -15.5 & -11.6 & -14.4 & -14.3 & -13.7 \\
Trade & -13.8 & -18.1 & -15.3 & -15.5 & -12.4 & -13.5 & -14.1 & -14.1 \\
Services & -15.8 & -19.8 & -12.5 & -13.6 & -10.8 & -11.6 & -13.0 & -12.7 \\
\hline Owner: Private & & & -15.6 & -14.2 & -11.5 & -12.4 & -14.2 & -13.9 \\
$\quad$ Government & -13.2 & -18.4 & -14.7 & -16.4 & -12.7 & -14.1 & -14.7 & -14.8 \\
$\quad$ Foreign & -8.8 & -17.4 & -9.4 & -12.3 & -8.9 & -11.1 & -11.8 & -11.8 \\
Imp. foreign & -7.6 & -21.4 & -12.0 & -12.9 & -10.8 & -11.3 & -12.8 & -12.9 \\
Other & -13.6 & -17.5 & -15.5 & -16.8 & -12.5 & -13.5 & -14.5 & -14.8 \\
\hline Size: Small & -13.5 & -17.1 & -14.5 & -15.7 & -12.1 & -13.1 & -14.2 & -14.3 \\
Medium & -13.8 & -18.4 & -16.0 & -16.4 & -11.9 & -13.0 & -14.2 & -14.3 \\
Large & -13.0 & -17.9 & -11.8 & -13.6 & -10.8 & -11.8 & -12.4 & -12.6 \\
\hline
\end{tabular}


Table 9-A: Single equation models: manufacturing

\begin{tabular}{|c|c|c|c|c|c|c|c|c|}
\hline Variable & 1990 & 1991 & 1992 & 1993 & 1994 & 1995 & 1996 & 1997 \\
\hline Constant & $0.54 * *$ & -0.09 & $0.33 * *$ & $0.54 * *$ & $0.39 * *$ & $0.20 * *$ & $0.12 * *$ & $0.20 * *$ \\
\hline $\mathrm{y}_{t-1}$ & $0.69 * *$ & $0.49 * *$ & $0.23 * *$ & $0.50 * *$ & $0.64 * *$ & $0.64 * *$ & $0.70 * *$ & $0.71 * *$ \\
\hline $\log (\mathrm{L})$ & $0.11 * *$ & $0.36 * *$ & $0.56 * *$ & $0.30 * *$ & $0.21 * *$ & $0.23 * *$ & $0.22 * *$ & $0.21 * *$ \\
\hline $\log (\mathrm{K})$ & $0.11 * *$ & $0.14 * *$ & $0.15 * *$ & $0.16 * *$ & $0.12 * *$ & $0.13 * *$ & $0.10 * *$ & $0.11 * *$ \\
\hline import penetration & $1.87 *$ & -0.42 & $-0.04 *$ & $-0.03 *$ & -0.01 & $0.03 * *$ & 0.01 & 0.00 \\
\hline market share sh $_{t}$ & -0.16 & $-0.64 * *$ & $1.28 * *$ & 0.32 & $0.51 * *$ & $0.33 * *$ & $0.25 * *$ & $0.12 * *$ \\
\hline concentration & $3.41 * *$ & 1.27 & 0.93 & 1.05 & 0.71 & -0.11 & $1.20 * *$ & -0.02 \\
\hline$\sigma$ & $0.87 * *$ & $1.03 * *$ & $0.86 * *$ & $0.87 * *$ & $0.67 * *$ & $0.66 * *$ & $0.64 * *$ & $0.67 * *$ \\
\hline$\sigma_{u} / \sigma_{v}$ & $2.13 * *$ & $2.70 * *$ & $1.94 * *$ & $1.89 * *$ & $1.61 * *$ & $1.15 * *$ & $1.20 * *$ & $1.68 * *$ \\
\hline short-run ret. to scale & $0.23 * *$ & $0.50 * *$ & $0.71 * *$ & $0.46 * *$ & $0.33 * *$ & $0.37 * *$ & $0.32 * *$ & $0.31 * *$ \\
\hline long-run ret. to scale & $0.74 * *$ & 0.98 & $0.93 * *$ & $0.91 * *$ & $0.92 * *$ & 1.01 & $1.07 * *$ & $1.09 * *$ \\
\hline Nob & 1182 & 668 & 1226 & 2244 & 2111 & 2991 & 3241 & 3629 \\
\hline Mean of dep.var & 4.01 & 3.80 & 3.72 & 3.63 & 3.90 & 3.63 & 3.52 & 3.39 \\
\hline S.dev of dep.var & 1.32 & 1.46 & 1.40 & 1.48 & 1.48 & 1.55 & 1.59 & 1.64 \\
\hline $\mathrm{R}^{2}$ & 0.77 & 0.76 & 0.81 & 0.82 & 0.88 & 0.88 & 0.90 & 0.91 \\
\hline Mean inefficiency (\%) & -14.99 & -19.48 & -16.28 & -16.38 & -11.28 & -10.86 & -10.89 & -13.06 \\
\hline
\end{tabular}

Table 9-B: Mean relative inefficiency for subsets of observations

\begin{tabular}{|l|c|c|c|c|c|c|c|c|}
\hline Variable & 1990 & 1991 & 1992 & 1993 & 1994 & 1995 & 1996 & 1997 \\
\hline Engineering & -15.2 & -22.3 & -15.6 & -15.4 & -10.9 & -9.9 & -10.0 & -11.7 \\
Chemical ind. & -14.2 & -16.5 & -12.8 & -13.8 & -9.7 & -11.2 & -10.4 & -12.8 \\
Pharmaceutical ind. & -10.0 & -11.2 & -11.4 & -15.7 & -8.0 & -11.7 & -12.1 & -12.1 \\
Food ind. & -13.3 & -15.9 & -14.1 & -17.5 & -13.2 & -11.4 & -12.6 & -15.4 \\
Light ind. & -14.9 & -19.5 & -18.1 & -17.5 & -11.4 & -11.5 & -11.1 & -13.5 \\
Other ind. & -17.3 & -17.6 & -15.9 & -15.9 & -10.6 & -10.6 & -11.0 & -13.1 \\
\hline Owner: Private & & & -15.1 & -15.4 & -11.1 & -10.4 & -11.3 & -13.2 \\
$\quad$ Government & -14.4 & -20.4 & -16.6 & -18.5 & -13.2 & -12.1 & -11.9 & -15.5 \\
$\quad$ Foreign & -13.8 & -18.9 & -9.9 & -13.0 & -8.7 & -9.9 & -9.4 & -11.4 \\
$\quad$ Imp. foreign & -7.9 & -16.0 & -12.5 & -13.7 & -10.8 & -10.0 & -10.6 & -11.9 \\
$\quad$ Other & -15.5 & -18.8 & -17.4 & -17.4 & -12.0 & -11.4 & -11.2 & -13.6 \\
\hline Size: Small & -15.0 & -19.7 & -16.1 & -16.9 & -11.4 & -11.0 & -11.0 & -13.2 \\
$\quad$ Medium & -14.8 & -19.5 & -17.2 & -16.0 & -11.2 & -10.8 & -10.7 & -13.0 \\
Large & -15.3 & -19.1 & -14.2 & -15.3 & -11.0 & -10.5 & -10.5 & -12.2 \\
\hline
\end{tabular}


Table 10-A: Two-equation models: manufacturing

\begin{tabular}{|c|c|c|c|c|c|c|c|c|}
\hline Variable & 1990 & 1991 & 1992 & 1993 & 1994 & 1995 & 1996 & 1997 \\
\hline \multicolumn{9}{|l|}{ Production function } \\
\hline Constant & $0.56 * *$ & 0.02 & 0.13 & $0.49 * *$ & $0.32 * *$ & $0.18 * *$ & $0.10 * *$ & $0.19 * *$ \\
\hline $\mathrm{y}_{t-1}$ & $0.70 * *$ & $0.49 * *$ & $0.24 * *$ & $0.51 * *$ & $0.65 * *$ & $0.64 * *$ & $0.71 * *$ & $0.72 * *$ \\
\hline $\log (\mathrm{L})$ & $0.10 * *$ & $0.33 * *$ & $0.59 * *$ & $0.30 * *$ & $0.22 * *$ & $0.24 * *$ & $0.22 * *$ & $0.20 * *$ \\
\hline $\log (\mathrm{K})$ & $0.13 * *$ & $0.15 * *$ & $0.15 * *$ & $0.17 * *$ & $0.12 * *$ & $0.13 * *$ & $0.10 * *$ & $0.11 * *$ \\
\hline$\sigma$ & $0.87 * *$ & $1.04 * *$ & $0.85 * *$ & $0.86 * *$ & $0.67 * *$ & $0.66 * *$ & $0.64 * *$ & $0.67 * *$ \\
\hline$\sigma_{u} / \sigma_{v}$ & $2.02 * *$ & $2.78 * *$ & $1.75 * *$ & $1.86 * *$ & $1.56 * *$ & $1.13 * *$ & $1.17 * *$ & $1.65 * *$ \\
\hline short-run ret. to scale & $0.23 * *$ & $0.48 * *$ & $0.75 * *$ & $0.47 * *$ & $0.34 * *$ & $0.37 * *$ & $0.32 * *$ & $0.31 * *$ \\
\hline long-run ret. to scale & $0.77 * *$ & 0.94 & 0.99 & $0.94 * *$ & 0.98 & 1.04 & $1.11 * *$ & $1.11 * *$ \\
\hline Nob & 1182 & 668 & 1226 & 2244 & 2111 & 2991 & 3241 & 3629 \\
\hline Mean of dep.var & 4.01 & 3.80 & 3.72 & 3.63 & 3.90 & 3.63 & 3.52 & 3.39 \\
\hline S.dev of dep.var & 1.32 & 1.46 & 1.40 & 1.48 & 1.48 & 1.55 & 1.59 & 1.64 \\
\hline $\mathrm{R}^{2}$ & 0.77 & 0.75 & 0.80 & 0.82 & 0.88 & 0.88 & 0.90 & 0.91 \\
\hline Mean inefficiency (\%) & -14.88 & -19.75 & -15.73 & -16.31 & -11.15 & -10.72 & -10.78 & -12.96 \\
\hline \multicolumn{9}{|l|}{ Market share equation } \\
\hline Constant & 0.00 & 0.01 & $0.02 * *$ & $0.01 * *$ & $0.01 * *$ & $0.01 * *$ & $0.01 * *$ & $0.02 * *$ \\
\hline market share $\operatorname{sh}_{t-1}$ & $0.89 * *$ & $1.06 * *$ & $0.43 * *$ & $0.95 * *$ & $1.00 * *$ & $1.02 * *$ & $0.85 * *$ & $0.24 * *$ \\
\hline efficiency & $0.44 * *$ & 1.32 & $1.94 * *$ & $1.12 * *$ & $1.75 * *$ & $1.62 * *$ & $1.84 * *$ & $1.61 * *$ \\
\hline import penetration & 0.00 & 0.02 & $-0.02 * *$ & $0.00 *$ & 0.00 & 0.00 & $-0.01 * *$ & $-0.01 * *$ \\
\hline concentration & 0.08 & -0.03 & $1.31 * *$ & 0.09 & -0.03 & -0.03 & 0.42 & $1.00 * *$ \\
\hline Mean of dep.var & 0.04 & 0.04 & 0.03 & 0.03 & 0.03 & 0.03 & 0.03 & 0.03 \\
\hline S.dev of dep.var & 0.11 & 0.12 & 0.08 & 0.10 & 0.11 & 0.10 & 0.10 & 0.10 \\
\hline SEE & 0.03 & 0.04 & 0.05 & 0.03 & 0.04 & 0.03 & 0.04 & 0.07 \\
\hline $\mathrm{R}^{2}$ & 0.94 & 0.90 & 0.53 & 0.91 & 0.88 & 0.93 & 0.79 & 0.44 \\
\hline White-hetero & $167 * *$ & $583 * *$ & $313 * *$ & $819 * *$ & $286 * *$ & $1452 * *$ & $359 * *$ & $2182 * *$ \\
\hline Reset $y^{2}$ & $84.73 * *$ & $175.41 * *$ & $4.29 *$ & $125.00 * *$ & $232.53 * *$ & $44.00 * *$ & $10.65 * *$ & $10655.00 * *$ \\
\hline Reset $\mathrm{y}^{2}, \mathrm{y}^{3}$ & $50.47 * *$ & $115.75 * *$ & $6.55 * *$ & $98.91 * *$ & $117.01 * *$ & $125.42 * *$ & $100.64 * *$ & $5452.52 * *$ \\
\hline \multicolumn{9}{|l|}{ Corr of rel. efficiency } \\
\hline $\begin{array}{l}\text { profit margin } \\
\text { investment rate }\end{array}$ & 0.34 & 0.56 & 0.37 & 0.46 & $\begin{array}{l}0.47 \\
0.19\end{array}$ & 0.41 & 0.44 & $\begin{array}{l}0.40 \\
0.21\end{array}$ \\
\hline
\end{tabular}

Table 10-B: Mean relative inefficiency for subsets of observations

\begin{tabular}{|l|c|c|c|c|c|c|c|c|}
\hline Variable & 1990 & 1991 & 1992 & 1993 & 1994 & 1995 & 1996 & 1997 \\
\hline Engineering & -15.1 & -22.6 & -15.3 & -15.4 & -10.7 & -9.7 & -9.9 & -11.6 \\
Chemical ind. & -14.1 & -17.4 & -11.9 & -13.8 & -9.6 & -11.0 & -10.2 & -12.7 \\
Pharmaceutical ind. & -10.2 & -12.6 & -10.7 & -15.7 & -8.2 & -11.7 & -12.1 & -12.1 \\
Food ind. & -13.1 & -15.8 & -13.9 & -17.2 & -13.0 & -11.4 & -12.6 & -15.3 \\
Light ind. & -14.9 & -19.7 & -17.5 & -17.5 & -11.3 & -11.3 & -11.0 & -13.4 \\
Other ind. & -16.5 & -17.7 & -15.0 & -15.6 & -10.5 & -10.6 & -10.9 & -13.0 \\
\hline Owner: Private & & & -15.0 & -15.3 & -11.0 & -10.3 & -11.2 & -13.2 \\
$\quad$ Government & -14.3 & -20.7 & -16.0 & -18.4 & -13.1 & -12.0 & -11.8 & -15.4 \\
$\quad$ Foreign & -11.8 & -19.4 & -9.9 & -12.9 & -8.7 & -9.7 & -9.3 & -11.3 \\
$\quad$ Imp. foreign & -8.4 & -16.1 & -12.3 & -13.7 & -10.7 & -9.9 & -10.4 & -11.9 \\
$\quad$ Other & -15.4 & -19.1 & -16.8 & -17.3 & -11.8 & -11.2 & -11.1 & -13.5 \\
\hline Size: Small & -14.7 & -20.1 & -15.4 & -16.7 & -11.2 & -10.8 & -10.9 & -13.1 \\
$\quad$ Medium & -14.7 & -19.7 & -17.0 & -16.1 & -11.2 & -10.7 & -10.7 & -13.0 \\
Large & -15.4 & -19.4 & -13.5 & -15.0 & -10.7 & -10.4 & -10.3 & -12.1 \\
\hline
\end{tabular}


Table 11: Two-equation models: engineering

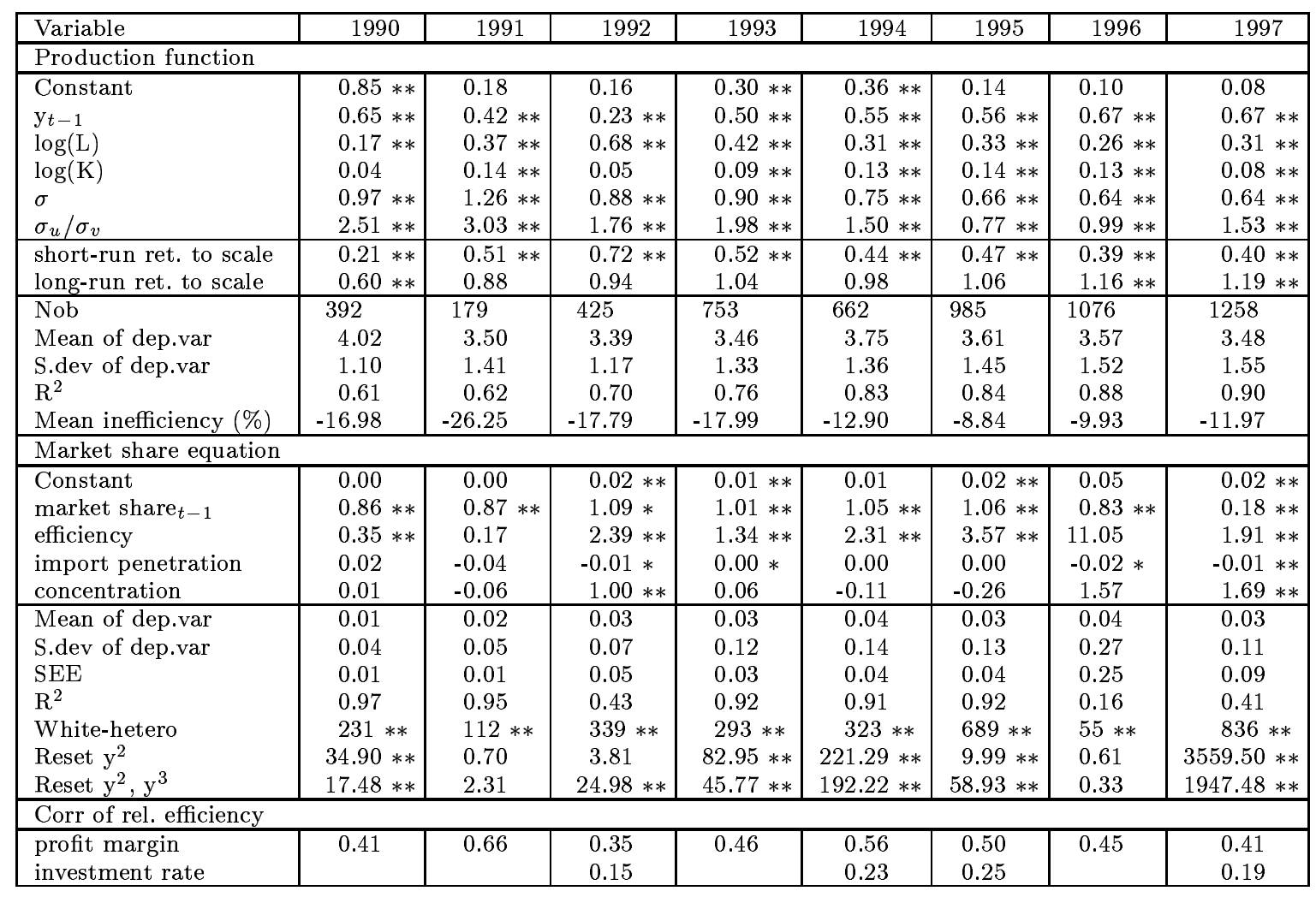

Table 12: Two-equation models: chemical industry

\begin{tabular}{|c|c|c|c|c|c|c|c|c|}
\hline Variable & 1990 & 1991 & 1992 & 1993 & 1994 & 1995 & 1996 & 1997 \\
\hline \multicolumn{9}{|l|}{ Production function } \\
\hline Constant & 0.68 & $0.48 * *$ & -0.09 & $0.54 *$ & $0.40 * *$ & 0.22 & 0.12 & $0.23 *$ \\
\hline $\mathrm{y}_{t-1}$ & $0.69 * *$ & $0.63 * *$ & $0.21 * *$ & $0.43 * *$ & $0.79 * *$ & $0.67 * *$ & $0.69 * *$ & $0.76 * *$ \\
\hline $\log (\mathrm{L})$ & 0.14 & -0.01 & $0.65 * *$ & $0.29 * *$ & $0.10 *$ & $0.15 * *$ & $0.17 * *$ & $0.13 * *$ \\
\hline $\log (K)$ & 0.12 & $0.30 * *$ & $0.22 * *$ & $0.25 * *$ & $0.10 * *$ & $0.19 * *$ & $0.16 * *$ & $0.14 * *$ \\
\hline$\sigma$ & $1.00 * *$ & $0.85 * *$ & $0.74 * *$ & $0.77 * *$ & $0.53 * *$ & $0.70 * *$ & $0.52 * *$ & $0.63 * *$ \\
\hline$\sigma_{u} / \sigma_{v}$ & $2.07 *$ & 246.63 & 1.41 & $1.57 * *$ & $1.19 * *$ & $1.41 * *$ & $0.86 *$ & $2.43 * *$ \\
\hline $\begin{array}{l}\text { short-run ret. to scale } \\
\text { long-run ret. to scale }\end{array}$ & $\begin{array}{l}0.26 * * \\
0.83\end{array}$ & $\begin{array}{l}0.29 * * \\
0.77 * *\end{array}$ & $\begin{array}{l}0.87 * \\
1.10\end{array}$ & $\begin{array}{l}0.54 * * \\
0.96\end{array}$ & $\begin{array}{l}0.20 * * \\
0.95\end{array}$ & $\begin{array}{l}0.34 * * \\
1.05\end{array}$ & $\begin{array}{l}0.33 * * \\
1.06\end{array}$ & $\begin{array}{l}0.27 * * \\
1.14\end{array}$ \\
\hline Nob & 72 & 43 & 88 & 174 & 173 & 243 & 263 & 283 \\
\hline Mean of dep.var & 4.97 & 4.31 & 4.28 & 4.18 & 4.47 & 4.09 & 3.92 & 3.80 \\
\hline S.dev of dep.var & 1.70 & 1.35 & 1.70 & 1.65 & 1.63 & 1.71 & 1.70 & 1.81 \\
\hline $\mathrm{R}^{2}$ & 0.83 & 0.85 & 0.89 & 0.88 & 0.93 & 0.90 & 0.93 & 0.94 \\
\hline Mean inefficiency (\%) & -14.24 & -14.31 & -11.31 & -12.31 & -7.10 & -10.87 & -6.82 & -11.49 \\
\hline \multicolumn{9}{|l|}{ Market share equation } \\
\hline Constant & 0.01 & -0.02 & 0.01 & 0.00 & 0.01 & $0.01 *$ & 0.00 & 0.00 \\
\hline market share $_{t-1}$ & $0.90 * *$ & $1.30 * *$ & $0.43 * *$ & $0.86 * *$ & $0.92 * *$ & $1.03 * *$ & $0.93 * *$ & $0.98 * *$ \\
\hline efficiency & 0.71 & -0.60 & $4.69 *$ & $0.49 *$ & $1.70 *$ & $2.07 *$ & 1.40 & $0.42 *$ \\
\hline import penetration & & 0.00 & $-0.01 * *$ & 0.00 & 0.00 & 0.00 & 0.00 & 0.00 \\
\hline concentration & -0.01 & 0.11 & $2.50 * *$ & 0.39 & -0.08 & -0.02 & $0.03 *$ & $0.17 * *$ \\
\hline Mean of dep.var & 0.11 & 0.12 & 0.05 & 0.04 & 0.04 & 0.04 & 0.03 & 0.03 \\
\hline S.dev of dep.var & 0.25 & 0.36 & 0.16 & 0.12 & 0.13 & 0.12 & 0.11 & 0.11 \\
\hline SEE & 0.08 & 0.05 & 0.04 & 0.03 & 0.02 & 0.03 & 0.01 & 0.01 \\
\hline $\mathrm{R}^{2}$ & 0.91 & 0.99 & 0.92 & 0.95 & 0.98 & 0.92 & 0.98 & 0.99 \\
\hline White-hetero & 16 & $38 * *$ & $76 * *$ & $73 * *$ & $132 * *$ & $39 * *$ & $173 * *$ & $152 * *$ \\
\hline Reset $\mathrm{y}^{2}$ & $12.94 * *$ & $6.93 *$ & $11.09 * *$ & $3.95 *$ & $4.79 *$ & 0.26 & 3.55 & 0.09 \\
\hline Reset $\mathrm{y}^{2}, \mathrm{y}^{3}$ & $7.04 * *$ & $132.34 * *$ & $8.70 * *$ & 2.42 & $5.17 * *$ & $6.78 * *$ & $11.84 * *$ & 0.38 \\
\hline \multicolumn{9}{|l|}{ Corr of rel. efficiency } \\
\hline $\begin{array}{l}\text { profit margin } \\
\text { investment rate }\end{array}$ & 0.26 & 0.38 & $\begin{array}{l}0.68 \\
0.20\end{array}$ & 0.43 & & 0.44 & $\begin{array}{l}0.52 \\
0.25\end{array}$ & $\begin{array}{l}0.44 \\
0.34\end{array}$ \\
\hline
\end{tabular}


Table 13: Two-equation models: food industry

\begin{tabular}{|c|c|c|c|c|c|c|c|c|}
\hline Variable & 1990 & 1991 & 1992 & 1993 & 1994 & 1995 & 1996 & 1997 \\
\hline \multicolumn{9}{|l|}{ Production function } \\
\hline Constant & -0.28 & -0.03 & 0.11 & $0.41 *$ & 0.06 & $0.21 *$ & -0.05 & 0.17 \\
\hline $\mathrm{y}_{t-1}$ & $0.44 * *$ & $0.49 * *$ & $0.18 * *$ & $0.33 * *$ & $0.51 * *$ & $0.59 * *$ & $0.70 * *$ & $0.55 * *$ \\
\hline $\log (\mathrm{L})$ & $0.64 * *$ & $0.66 *$ & $0.63 * *$ & $0.42 * *$ & $0.36 * *$ & $0.24 * *$ & $0.20 * *$ & $0.26 * *$ \\
\hline $\log (\mathrm{K})$ & -0.06 & -0.20 & $0.20 * *$ & $0.21 * *$ & $0.16 * *$ & $0.16 * *$ & $0.14 * *$ & $0.22 * *$ \\
\hline$\sigma$ & $0.84 * *$ & $0.92 * *$ & $0.78 * *$ & $0.91 * *$ & $0.82 * *$ & $0.68 * *$ & $0.66 * *$ & $0.85 * *$ \\
\hline$\sigma_{u} / \sigma_{v}$ & 4.49 & 3.49 & $2.35 * *$ & $2.64 * *$ & $2.62 * *$ & $1.68 * *$ & $1.68 * *$ & $2.28 * *$ \\
\hline $\begin{array}{l}\text { short-run ret. to scale } \\
\text { long-run ret. to scale }\end{array}$ & $\begin{array}{l}0.58 * * \\
1.03\end{array}$ & $\begin{array}{l}0.46 * * \\
0.90\end{array}$ & $\begin{array}{l}0.83 * * \\
1.00\end{array}$ & $\begin{array}{l}0.63 * * \\
0.94\end{array}$ & $\begin{array}{l}0.52 * * \\
1.06\end{array}$ & $\begin{array}{l}0.40 * * \\
0.99\end{array}$ & $\begin{array}{l}0.34 * * \\
1.15 *\end{array}$ & $\begin{array}{l}0.47 * * \\
1.05\end{array}$ \\
\hline Nob & 97 & 65 & 129 & 274 & 304 & 460 & 487 & 531 \\
\hline Mean of dep.var & 5.08 & 4.87 & 4.56 & 4.09 & 4.19 & 3.70 & 3.46 & 3.18 \\
\hline S.dev of dep.var & 1.05 & 1.09 & 1.48 & 1.59 & 1.60 & 1.68 & 1.76 & 1.81 \\
\hline $\mathrm{R}^{2}$ & 0.69 & 0.66 & 0.87 & 0.84 & 0.87 & 0.91 & 0.92 & 0.89 \\
\hline Mean inefficiency (\%) & -11.85 & -13.22 & -12.33 & -15.74 & -13.82 & -12.17 & -12.85 & -18.46 \\
\hline \multicolumn{9}{|l|}{ Market share equation } \\
\hline Constant & -0.01 & 0.00 & 0.01 & 0.00 & 0.00 & $0.00 * *$ & $0.00 *$ & $0.00 *$ \\
\hline market share $_{t-1}$ & $0.79 * *$ & $1.00 * *$ & $0.88 * *$ & $0.84 * *$ & $0.98 * *$ & $1.02 * *$ & $0.99 * *$ & $0.95 * *$ \\
\hline efficiency & 0.00 & 0.10 & $0.86 *$ & $1.23 * *$ & $0.70 * *$ & $0.58 * *$ & $0.54 * *$ & $0.70 *$ \\
\hline import penetration & & 0.00 & $0.16 *$ & 0.13 & -0.01 & 0.00 & -0.02 & 0.03 \\
\hline concentration & $0.48 *$ & 0.27 & 0.18 & 0.04 & 0.04 & 0.10 & 0.12 & -0.09 \\
\hline Mean of dep.var & 0.07 & 0.05 & 0.03 & 0.04 & 0.04 & 0.03 & 0.03 & 0.03 \\
\hline S.dev of dep.var & 0.15 & 0.06 & 0.05 & 0.10 & 0.10 & 0.09 & 0.09 & 0.08 \\
\hline SEE & 0.03 & 0.01 & 0.04 & 0.03 & 0.02 & 0.01 & 0.02 & 0.02 \\
\hline $\mathrm{R}^{2}$ & 0.96 & 0.97 & 0.53 & 0.91 & 0.97 & 0.98 & 0.97 & 0.95 \\
\hline White-hetero & $68 * *$ & $27 * *$ & 16 & $189 * *$ & $127 * *$ & $155 * *$ & $105 * *$ & $277 * *$ \\
\hline Reset $\mathrm{y}^{2}$ & $36.26 * *$ & $17.21 * *$ & 0.07 & 0.56 & $8.81 * *$ & 0.26 & $6.53 *$ & 1.66 \\
\hline Reset $\mathrm{y}^{2}, \mathrm{y}^{3}$ & $36.34 * *$ & $13.69 * *$ & 0.58 & $3.22 *$ & $8.47 * *$ & 0.22 & $11.12 * *$ & $10.14 * *$ \\
\hline \multicolumn{9}{|l|}{ Corr of rel. efficiency } \\
\hline $\begin{array}{l}\text { profit margin } \\
\text { investment rate }\end{array}$ & 0.33 & $\begin{array}{r}0.38 \\
-0.16\end{array}$ & $\begin{array}{l}0.64 \\
0.15\end{array}$ & $\begin{array}{l}0.32 \\
0.55\end{array}$ & $\begin{array}{l}0.43 \\
0.27\end{array}$ & $\overline{0.41}$ & 0.30 & $\begin{array}{l}0.43 \\
0.30\end{array}$ \\
\hline
\end{tabular}

Table 14: Two-equation models: light industry

\begin{tabular}{|c|c|c|c|c|c|c|c|c|}
\hline Variable & 1990 & 1991 & 1992 & 1993 & 1994 & 1995 & 1996 & 1997 \\
\hline \multicolumn{9}{|l|}{ Production function } \\
\hline Constant & $0.37 * *$ & -0.06 & 0.05 & $0.35 * *$ & $0.28 * *$ & 0.09 & 0.09 & $0.25 * *$ \\
\hline $\mathrm{y}_{t-1}$ & $0.69 * *$ & $0.53 * *$ & $0.22 * *$ & $0.61 * *$ & $0.77 * *$ & $0.66 * *$ & $0.69 * *$ & $0.75 * *$ \\
\hline $\log (\mathrm{L})$ & $0.10 * *$ & $0.33 * *$ & $0.57 * *$ & $0.23 * *$ & $0.12 * *$ & $0.24 * *$ & $0.24 * *$ & $0.15 * *$ \\
\hline $\log (\mathrm{K})$ & $0.15 * *$ & $0.12 * *$ & $0.20 * *$ & $0.16 * *$ & $0.10 * *$ & $0.12 * *$ & $0.09 * *$ & $0.11 * *$ \\
\hline$\sigma$ & $0.68 * *$ & $0.97 * *$ & $0.83 * *$ & $0.80 * *$ & $0.53 * *$ & $0.64 * *$ & $0.62 * *$ & $0.63 * *$ \\
\hline$\sigma_{u} / \sigma_{v}$ & $1.57 * *$ & $2.74 * *$ & $2.03 * *$ & $1.72 * *$ & $1.51 * *$ & $1.54 * *$ & $1.35 * *$ & $1.94 * *$ \\
\hline $\begin{array}{l}\text { short-run ret. to scale } \\
\text { long-run ret. to scale }\end{array}$ & $\begin{array}{l}0.25 * * \\
0.80 * *\end{array}$ & $\begin{array}{l}0.45 * * \\
0.95\end{array}$ & $\begin{array}{l}0.77 * * \\
0.98\end{array}$ & $\begin{array}{l}0.39 * * \\
0.99\end{array}$ & $\begin{array}{l}0.23 * * \\
0.98\end{array}$ & $\begin{array}{l}0.36 * * \\
1.06\end{array}$ & $\begin{array}{l}0.32 * * \\
1.06\end{array}$ & $\begin{array}{l}0.26 * * \\
1.06\end{array}$ \\
\hline Nob & 537 & 309 & 483 & 843 & 764 & 988 & 1065 & 1173 \\
\hline Mean of dep.var & 3.51 & 3.29 & 3.43 & 3.29 & 3.54 & 3.27 & 3.18 & 3.06 \\
\hline S.dev of dep.var & 1.07 & 1.14 & 1.19 & 1.29 & 1.26 & 1.36 & 1.37 & 1.43 \\
\hline $\mathrm{R}^{2}$ & 0.77 & 0.65 & 0.76 & 0.79 & 0.90 & 0.87 & 0.88 & 0.90 \\
\hline Mean inefficiency (\%) & -12.71 & -21.23 & -17.08 & -16.31 & -9.72 & -12.73 & -12.18 & -14.05 \\
\hline \multicolumn{9}{|l|}{ Market share equation } \\
\hline Constant & $0.00 * *$ & 0.01 & $0.02 * *$ & $0.01 * *$ & $0.01 *$ & $0.00 * *$ & $0.01 * *$ & $0.01 * *$ \\
\hline market share $_{t-1}$ & $0.84 * *$ & $0.49 * *$ & $0.50 * *$ & $0.95 * *$ & $0.82 * *$ & $1.00 * *$ & $0.65 * *$ & $0.93 * *$ \\
\hline efficiency & 0.38 & $0.92 *$ & $0.95 *$ & $0.84 * *$ & $1.32 *$ & $0.87 * *$ & $2.22 * *$ & $1.05 * *$ \\
\hline import penetration & & -0.05 & $-0.03 * *$ & 0.00 & 0.00 & 0.00 & $-0.02 * *$ & $0.00 *$ \\
\hline concentration & -0.06 & $1.67 *$ & $2.01 * *$ & 0.21 & 0.42 & 0.03 & $2.89 * *$ & $0.27 *$ \\
\hline Mean of dep.var & 0.02 & 0.02 & 0.02 & 0.02 & 0.02 & 0.02 & 0.02 & 0.02 \\
\hline S.dev of dep.var & 0.05 & 0.04 & 0.07 & 0.04 & 0.06 & 0.05 & 0.08 & 0.07 \\
\hline SEE & 0.01 & 0.03 & 0.05 & 0.02 & 0.04 & 0.02 & 0.05 & 0.01 \\
\hline $\mathrm{R}^{2}$ & 0.94 & 0.58 & 0.51 & 0.81 & 0.60 & 0.92 & 0.62 & 0.96 \\
\hline White-hetero & $178 * *$ & $222 * *$ & $135 * *$ & $104 * *$ & $179 * *$ & $339 * *$ & $399 * *$ & $507 * *$ \\
\hline Reset $\mathrm{y}^{2}$ & $17.15 * *$ & $306.88 * *$ & $26.34 * *$ & 2.53 & $60.80 * *$ & $33.29 * *$ & $96.22 * *$ & $103.45 * *$ \\
\hline Reset $\mathrm{y}^{2}, \mathrm{y}^{3}$ & $12.41 * *$ & $247.00 * *$ & $18.72 * *$ & 1.27 & $81.69 * *$ & $19.92 * *$ & $78.29 * *$ & $111.32 * *$ \\
\hline \multicolumn{9}{|l|}{ Corr of rel. efficiency } \\
\hline $\begin{array}{l}\text { profit margin } \\
\text { investment rate }\end{array}$ & 0.31 & 0.48 & $\begin{array}{l}0.50 \\
0.17\end{array}$ & 0.54 & 0.43 & 0.41 & $\begin{array}{l}0.48 \\
0.19\end{array}$ & $\begin{array}{l}0.48 \\
0.19\end{array}$ \\
\hline
\end{tabular}


Table 15: Two-equation models: other industries

\begin{tabular}{|c|c|c|c|c|c|c|c|c|}
\hline Variable & 1990 & 1991 & 1992 & 1993 & 1994 & 1995 & 1996 & 1997 \\
\hline \multicolumn{9}{|l|}{ Production function } \\
\hline Constant & 0.58 & 0.24 & 0.22 & $0.66 * *$ & $0.28 *$ & $0.24 * *$ & 0.13 & 0.05 \\
\hline $\mathrm{y}_{t-1}$ & $0.73 * *$ & $0.37 * *$ & $0.21 * *$ & $0.37 * *$ & $0.56 * *$ & $0.65 * *$ & $0.64 * *$ & $0.68 * *$ \\
\hline $\log (\mathrm{L})$ & 0.03 & 0.33 & $0.63 * *$ & $0.39 * *$ & $0.34 * *$ & $0.24 * *$ & $0.28 * *$ & $0.22 * *$ \\
\hline $\log (\mathrm{K})$ & $0.20 *$ & $0.21 *$ & $0.13 * *$ & $0.17 * *$ & $0.10 * *$ & $0.12 * *$ & $0.09 * *$ & $0.13 * *$ \\
\hline$\sigma$ & $1.14 * *$ & $0.88 * *$ & $0.83 * *$ & $0.84 * *$ & $0.59 * *$ & $0.65 * *$ & $0.61 * *$ & $0.52 * *$ \\
\hline$\sigma_{u} / \sigma_{v}$ & $2.06 * *$ & 2.01 & $2.13 * *$ & $2.41 * *$ & $1.86 * *$ & $1.88 * *$ & $1.60 * *$ & $1.08 * *$ \\
\hline $\begin{array}{l}\text { short-run ret. to scale } \\
\text { long-run ret. to scale }\end{array}$ & $\begin{array}{l}0.23 * * \\
0.86\end{array}$ & $\begin{array}{l}0.54 * * \\
0.86\end{array}$ & $\begin{array}{l}0.76 * * \\
0.97\end{array}$ & $\begin{array}{l}0.56 * * \\
0.89 *\end{array}$ & $\begin{array}{l}0.43 * * \\
0.99\end{array}$ & $\begin{array}{l}0.36 * * \\
1.03\end{array}$ & $\begin{array}{l}0.37 * * \\
1.05\end{array}$ & $\begin{array}{l}0.35 * * \\
1.09\end{array}$ \\
\hline Nob & 98 & 78 & 114 & 238 & 251 & 369 & 412 & 449 \\
\hline Mean of dep.var & 5.44 & 5.50 & 4.90 & 4.51 & 4.71 & 4.39 & 4.20 & 4.10 \\
\hline S.dev of dep.var & 1.78 & 1.40 & 1.66 & 1.72 & 1.64 & 1.71 & 1.74 & 1.79 \\
\hline $\mathrm{R}^{2}$ & 0.79 & 0.80 & 0.87 & 0.88 & 0.93 & 0.92 & 0.93 & 0.94 \\
\hline Mean inefficiency (\%) & -14.45 & -11.11 & -12.04 & -13.20 & -8.64 & -9.98 & -9.47 & -7.27 \\
\hline \multicolumn{9}{|l|}{ Market share equation } \\
\hline Constant & 0.00 & 0.01 & $0.04 * *$ & 0.01 & $0.01 *$ & $0.02 *$ & $0.02 *$ & 0.01 \\
\hline market share ${ }_{t-1}$ & $0.98 * *$ & $1.01 * *$ & $0.39 * *$ & $1.02 * *$ & $1.00 * *$ & $0.85 * *$ & $0.65 * *$ & $1.08 * *$ \\
\hline efficiency & 0.81 & $2.42 *$ & 2.18 & $1.98 *$ & $2.83 *$ & $1.93 *$ & 2.51 & $2.51 *$ \\
\hline import penetration & -0.02 & 0.00 & $-0.01 * *$ & 0.00 & 0.00 & 0.00 & $0.00 *$ & 0.00 \\
\hline concentration & 0.15 & 0.03 & $0.66 * *$ & 0.03 & 0.04 & -0.08 & 0.27 & -0.01 \\
\hline Mean of dep.var & 0.12 & 0.09 & 0.08 & 0.06 & 0.07 & 0.05 & 0.04 & 0.04 \\
\hline S.dev of dep.var & 0.19 & 0.18 & 0.12 & 0.13 & 0.14 & 0.11 & 0.09 & 0.11 \\
\hline SEE & 0.05 & 0.04 & 0.08 & 0.04 & 0.05 & 0.04 & 0.04 & 0.03 \\
\hline $\mathrm{R}^{2}$ & 0.93 & 0.96 & 0.58 & 0.90 & 0.87 & 0.87 & 0.81 & 0.95 \\
\hline White-hetero & $60 * *$ & $54 * *$ & 17 & $146 * *$ & $43 * *$ & $336 * *$ & $379 * *$ & $378 * *$ \\
\hline Reset $\mathrm{y}^{2}$ & $12.33 * *$ & $16.49 * *$ & 1.22 & $29.89 * *$ & 3.50 & $43.01 * *$ & $329.47 * *$ & $95.45 * *$ \\
\hline Reset $\mathrm{y}^{2}, \mathrm{y}^{3}$ & $6.12 * *$ & $13.97 * *$ & 1.31 & $21.23 * *$ & $3.18 *$ & $21.48 * *$ & $292.55 * *$ & $87.23 * *$ \\
\hline \multicolumn{9}{|l|}{ Corr of rel. efficiency } \\
\hline $\begin{array}{l}\text { profit margin } \\
\text { investment rate }\end{array}$ & 0.36 & 0.62 & 0.37 & $\begin{array}{l}0.62 \\
0.17\end{array}$ & 0.45 & $\begin{array}{l}0.37 \\
0.21\end{array}$ & 0.48 & $\begin{array}{l}0.35 \\
0.23\end{array}$ \\
\hline
\end{tabular}

Table 16: Two-equation models: agriculture

\begin{tabular}{|c|c|c|c|c|c|c|}
\hline Variable & 1992 & 1993 & 1994 & 1995 & 1996 & 1997 \\
\hline \multicolumn{7}{|l|}{ Production function } \\
\hline Constant & -0.08 & 0.03 & $-0.16 *$ & 0.04 & $0.16 *$ & 0.00 \\
\hline $\mathrm{y}_{t-1}$ & $0.19 * *$ & $0.32 * *$ & $0.43 * *$ & $0.57 * *$ & $0.66 * *$ & $0.58 * *$ \\
\hline $\log (\mathrm{L})$ & $0.67 * *$ & $0.56 * *$ & $0.55 * *$ & $0.37 * *$ & $0.21 * *$ & $0.33 * *$ \\
\hline $\log (K)$ & $0.08 * *$ & $0.05 * *$ & $0.03 * *$ & $0.04 * *$ & $0.11 * *$ & $0.08 * *$ \\
\hline$\sigma$ & $0.81 * *$ & $0.88 * *$ & $0.62 * *$ & $0.63 * *$ & $0.75 * *$ & $0.75 * *$ \\
\hline$\sigma_{u} / \sigma_{v}$ & $2.60 * *$ & $2.39 * *$ & $1.86 * *$ & $1.64 * *$ & $2.31 * *$ & $1.97 * *$ \\
\hline short-run ret. to scale & $0.75 * *$ & $0.62 * *$ & $0.58 * *$ & $0.41 * *$ & $0.33 * *$ & $0.41 * *$ \\
\hline long-run ret. to scale & $0.93 * *$ & $0.91 * *$ & 1.03 & 0.96 & 0.96 & 0.99 \\
\hline Nob & 799 & 1072 & 1107 & 1396 & 1459 & 1509 \\
\hline Mean of dep.var & 3.74 & 3.27 & 3.45 & 3.23 & 3.01 & 2.85 \\
\hline S.dev of dep.var & 0.95 & 1.04 & 1.04 & 1.10 & 1.20 & 1.21 \\
\hline $\mathrm{R}^{2}$ & 0.66 & 0.64 & 0.81 & 0.82 & 0.80 & 0.80 \\
\hline Mean inefficiency (\%) & -15.69 & -19.30 & -12.38 & -12.91 & -17.57 & -18.21 \\
\hline \multicolumn{7}{|l|}{ Market share equation } \\
\hline Constant & 0.00 & $0.00 *$ & 0.00 & 0.00 & 0.00 & $0.00 * *$ \\
\hline market share $_{t-1}$ & $0.58 * *$ & $0.81 * *$ & $0.86 * *$ & $1.08 * *$ & 0.32 & $0.82 * *$ \\
\hline efficiency & $0.16 * *$ & $0.09 * *$ & $0.21 * *$ & $0.05 *$ & $0.19 * *$ & $0.09 * *$ \\
\hline import penetration & 0.01 & $0.00 * *$ & 0.00 & $0.00 *$ & -0.01 & $0.00 *$ \\
\hline concentration & $3.15 *$ & $0.38 *$ & 1.65 & $0.48 *$ & 2.52 & -0.26 \\
\hline Mean of dep.var & 0.00 & 0.00 & 0.00 & 0.00 & 0.00 & 0.00 \\
\hline S.dev of dep.var & 0.01 & 0.01 & 0.01 & 0.01 & 0.01 & 0.01 \\
\hline $\mathrm{SEE}$ & 0.01 & 0.00 & 0.01 & 0.00 & 0.01 & 0.00 \\
\hline $\mathrm{R}^{2}$ & 0.65 & 0.92 & 0.76 & 0.95 & 0.53 & 0.94 \\
\hline White-hetero & $372 * *$ & $480 * *$ & $353 * *$ & $865 * *$ & $1405 * *$ & $462 * *$ \\
\hline Reset $\mathrm{y}^{2}$ & $79.19 * *$ & $35.61 * *$ & $23.64 * *$ & $615.46 * *$ & $974.40 * *$ & $515.25 * *$ \\
\hline Reset $\mathrm{y}^{2}, \mathrm{y}^{3}$ & $39.61 * *$ & $21.27 * *$ & $28.31 * *$ & $445.36 * *$ & $1538.57 * *$ & $272.75 * *$ \\
\hline \multicolumn{7}{|l|}{ Corr of rel. efficiency } \\
\hline $\begin{array}{l}\text { profit margin } \\
\text { investment rate }\end{array}$ & 0.47 & 0.35 & $\begin{array}{l}0.33 \\
0.18\end{array}$ & 0.18 & $\begin{array}{l}0.16 \\
0.17\end{array}$ & 0.23 \\
\hline
\end{tabular}


Table 17: Two-equation models: construction

\begin{tabular}{|c|c|c|c|c|c|c|c|c|}
\hline Variable & 1990 & 1991 & 1992 & 1993 & 1994 & 1995 & 1996 & 1997 \\
\hline \multicolumn{9}{|l|}{ Production function } \\
\hline Constant & $0.72 * *$ & 0.45 & 0.16 & 0.04 & $-0.19 *$ & -0.09 & $0.25 * *$ & 0.02 \\
\hline $\mathrm{y}_{t-1}$ & $0.67 * *$ & $0.81 * *$ & $0.14 * *$ & $0.43 * *$ & $0.63 * *$ & $0.60 * *$ & $0.65 * *$ & $0.54 * *$ \\
\hline $\log (\mathrm{L})$ & $0.14 *$ & 0.08 & $0.73 * *$ & $0.54 * *$ & $0.40 * *$ & $0.41 * *$ & $0.29 * *$ & $0.38 * *$ \\
\hline $\log (K)$ & 0.06 & 0.00 & 0.04 & 0.03 & $0.04 * *$ & 0.02 & $0.06 * *$ & $0.11 * *$ \\
\hline$\sigma$ & $0.81 * *$ & $0.94 * *$ & $0.96 * *$ & $0.87 * *$ & $0.59 * *$ & $0.81 * *$ & $0.83 * *$ & $0.70 * *$ \\
\hline$\sigma_{u} / \sigma_{v}$ & $1.42 * *$ & $2.40 * *$ & $1.84 * *$ & $1.48 * *$ & $1.59 * *$ & $1.83 * *$ & $1.89 * *$ & $1.24 * *$ \\
\hline short-run ret. to scale & $0.20 * *$ & $0.08 * *$ & $0.77 * *$ & $0.57 * *$ & $0.44 * *$ & $0.42 * *$ & $0.34 * *$ & $0.49 * *$ \\
\hline long-run ret. to scale & $0.59 * *$ & $0.44 *$ & $0.90 *$ & 1.00 & $1.19 * *$ & 1.06 & 0.99 & $1.08 *$ \\
\hline Nob & 341 & 153 & 416 & 763 & 586 & 896 & 933 & 1039 \\
\hline Mean of dep.var & 3.99 & 3.52 & 3.08 & 3.05 & 3.23 & 2.77 & 2.65 & 2.56 \\
\hline S.dev of dep.var & 1.08 & 1.06 & 1.05 & 1.13 & 1.14 & 1.23 & 1.25 & 1.25 \\
\hline $\mathrm{R}^{2}$ & 0.66 & 0.62 & 0.56 & 0.66 & 0.85 & 0.77 & 0.77 & 0.81 \\
\hline Mean inefficiency (\%) & -12.93 & -19.26 & -21.64 & -18.51 & -12.02 & -20.04 & -21.31 & -16.56 \\
\hline \multicolumn{9}{|l|}{ Market share equation } \\
\hline Constant & 0.00 & -0.01 & $0.00 *$ & $0.00 * *$ & $0.00 * *$ & $0.00 *$ & $0.00 * *$ & $0.00 * *$ \\
\hline market share $_{t-1}$ & $0.76 * *$ & $1.02 * *$ & 0.03 & $0.68 * *$ & 0.19 & $1.00 * *$ & $0.88 * *$ & $1.02 * *$ \\
\hline efficiency & 0.35 & 0.77 & $0.25 * *$ & $0.23 * *$ & $0.43 * *$ & $0.17 * *$ & $0.11 * *$ & $0.17 * *$ \\
\hline import penetration & 0.02 & $-0.71 *$ & 0.00 & 0.00 & 0.00 & 0.00 & 0.00 & 0.00 \\
\hline concentration & 0.93 & $3.92 *$ & $3.17 * *$ & 0.85 & 1.40 & 0.31 & 0.30 & 0.40 \\
\hline Mean of dep.var & 0.02 & 0.02 & 0.00 & 0.00 & 0.00 & 0.00 & 0.00 & 0.00 \\
\hline S.dev of dep.var & 0.04 & 0.08 & 0.01 & 0.01 & 0.01 & 0.01 & 0.01 & 0.01 \\
\hline SEE & 0.01 & 0.02 & 0.01 & 0.00 & 0.01 & 0.00 & 0.00 & 0.00 \\
\hline $\mathrm{R}^{2}$ & 0.87 & 0.92 & 0.15 & 0.69 & 0.17 & 0.84 & 0.88 & 0.91 \\
\hline White-hetero & $173 * *$ & $140 * *$ & $197 * *$ & $634 * *$ & $370 * *$ & $231 * *$ & $466 * *$ & $131 * *$ \\
\hline Reset $\mathrm{y}^{2}$ & 0.14 & $435.98 * *$ & $187.72 * *$ & $134.19 * *$ & $2441.18 * *$ & $15.93 * *$ & 1.53 & $21.53 * *$ \\
\hline Reset $\mathrm{y}^{2}, \mathrm{y}^{3}$ & $10.71 * *$ & $232.64 * *$ & $93.81 * *$ & $227.69 * *$ & $1983.21 * *$ & $15.02 * *$ & $5.27 * *$ & $18.02 * *$ \\
\hline \multicolumn{9}{|l|}{ Corr of rel. efficiency } \\
\hline $\begin{array}{l}\text { profit margin } \\
\text { investment rate }\end{array}$ & 0.59 & 0.63 & $\begin{array}{l}0.70 \\
0.28\end{array}$ & $\begin{array}{l}0.64 \\
0.17\end{array}$ & $\begin{array}{l}0.55 \\
0.16\end{array}$ & 0.50 & 0.44 & 0.42 \\
\hline
\end{tabular}

Table 18: Two-equation models: trade

\begin{tabular}{|c|c|c|c|c|c|c|c|c|}
\hline Variable & 1990 & 1991 & 1992 & 1993 & 1994 & 1995 & 1996 & 1997 \\
\hline \multicolumn{9}{|l|}{ Production function } \\
\hline Constant & 0.05 & -0.02 & -0.14 & $0.23 * *$ & $0.27 * *$ & $0.14 * *$ & $0.15 * *$ & $0.11 * *$ \\
\hline $\mathrm{y}_{t-1}$ & $0.64 * *$ & $0.73 * *$ & $0.22 * *$ & $0.63 * *$ & $0.78 * *$ & $0.75 * *$ & $0.77 * *$ & $0.79 * *$ \\
\hline $\log (\mathrm{L})$ & $0.24 * *$ & $0.16 * *$ & $0.63 * *$ & $0.28 * *$ & $0.16 * *$ & $0.18 * *$ & $0.18 * *$ & $0.17 * *$ \\
\hline $\log (\mathrm{K})$ & $0.11 * *$ & $0.08 * *$ & $0.16 * *$ & $0.07 * *$ & $0.07 * *$ & $0.10 * *$ & $0.08 * *$ & $0.08 * *$ \\
\hline$\sigma$ & $0.77 * *$ & $0.85 * *$ & $0.88 * *$ & $0.80 * *$ & $0.75 * *$ & $0.77 * *$ & $0.73 * *$ & $0.70 * *$ \\
\hline$\sigma_{u} / \sigma_{v}$ & $1.55 * *$ & $1.84 * *$ & $1.02 * *$ & $0.80 * *$ & $1.54 * *$ & $1.26 * *$ & $1.25 * *$ & $1.11 * *$ \\
\hline short-run ret. to scale & $0.35 * *$ & $0.24 * *$ & $0.78 * *$ & $0.35 * *$ & $0.23 * *$ & $0.28 * *$ & $0.26 * *$ & $0.26 * *$ \\
\hline long-run ret. to scale & 0.95 & 0.89 & 1.00 & 0.95 & 1.07 & $1.11 *$ & $1.13 * *$ & $1.19 * *$ \\
\hline Nob & 574 & 537 & 915 & 1886 & 1585 & 2315 & 2602 & 3030 \\
\hline Mean of dep.var & 4.63 & 3.89 & 3.55 & 3.39 & 3.55 & 3.17 & 3.03 & 2.85 \\
\hline S.dev of dep.var & 1.12 & 1.09 & 1.34 & 1.37 & 1.40 & 1.46 & 1.46 & 1.51 \\
\hline $\mathrm{R}^{2}$ & 0.73 & 0.67 & 0.71 & 0.74 & 0.84 & 0.83 & 0.84 & 0.86 \\
\hline Mean inefficiency (\%) & -10.81 & -14.94 & -14.14 & -11.74 & -13.66 & -14.88 & -14.84 & -14.29 \\
\hline \multicolumn{9}{|l|}{ Market share equation } \\
\hline Constant & 0.00 & 0.00 & 0.01 & -0.01 & $0.02 * *$ & $0.01 * *$ & $0.01 *$ & $0.01 * *$ \\
\hline market share $_{t-1}$ & $0.82 * *$ & $0.66 * *$ & $0.90 * *$ & 0.33 & $0.57 * *$ & $0.77 * *$ & $0.83 * *$ & $0.86 * *$ \\
\hline efficiency & $0.94 *$ & 0.65 & $1.26 * *$ & $2.38 *$ & $1.51 * *$ & $1.29 * *$ & $1.49 * *$ & $1.04 * *$ \\
\hline import penetration & & $-0.09 * *$ & 0.00 & 0.00 & 0.00 & 0.00 & 0.00 & 0.00 \\
\hline concentration & 0.65 & $1.26 * *$ & $2.69 * *$ & $6.94 *$ & -0.12 & 0.48 & 0.70 & 0.17 \\
\hline Mean of dep.var & 0.03 & 0.02 & 0.02 & 0.02 & 0.02 & 0.02 & 0.02 & 0.01 \\
\hline S.dev of dep.var & 0.08 & 0.06 & 0.07 & 0.10 & 0.07 & 0.06 & 0.05 & 0.04 \\
\hline SEE & 0.02 & 0.02 & 0.04 & 0.06 & 0.03 & 0.03 & 0.02 & 0.02 \\
\hline $\mathrm{R}^{2}$ & 0.92 & 0.86 & 0.62 & 0.65 & 0.81 & 0.81 & 0.84 & 0.83 \\
\hline White-hetero & $445 * *$ & $519 * *$ & $349 * *$ & $1864 * *$ & $861 * *$ & $766 * *$ & $1228 * *$ & $1157 * *$ \\
\hline Reset $\mathrm{y}^{2}$ & $18.93 * *$ & $5.10 *$ & 0.76 & $9148.01 * *$ & $1610.22 * *$ & $396.01 * *$ & $24.91 * *$ & 0.00 \\
\hline Reset $\mathrm{y}^{2}, \mathrm{y}^{3}$ & $10.63 * *$ & $160.49 * *$ & $13.12 * *$ & $7473.81 * *$ & $807.38 * *$ & $197.95 * *$ & $50.20 * *$ & $64.34 * *$ \\
\hline \multicolumn{9}{|l|}{ Corr of rel. efficiency } \\
\hline $\begin{array}{l}\text { profit margin } \\
\text { investment rate }\end{array}$ & 0.29 & 0.28 & $\begin{array}{l}0.17 \\
0.19\end{array}$ & 0.16 & & 0.17 & & \\
\hline
\end{tabular}


Table 19: Two-equation models: services

\begin{tabular}{|c|c|c|c|c|c|c|c|c|}
\hline Variable & 1990 & 1991 & 1992 & 1993 & 1994 & 1995 & 1996 & 1997 \\
\hline \multicolumn{9}{|l|}{ Production function } \\
\hline Constant & 0.03 & -0.68 & 0.39 & -0.09 & $0.44 * *$ & 0.12 & $0.27 * *$ & $0.29 * *$ \\
\hline $\mathrm{y}_{t-1}$ & $0.63 * *$ & 0.15 & $0.28 * *$ & $0.58 * *$ & $0.79 * *$ & $0.75 * *$ & $0.75 * *$ & $0.65 * *$ \\
\hline $\log (\mathrm{L})$ & 0.08 & 0.57 & $0.36 * *$ & $0.39 * *$ & $0.16 * *$ & $0.17 * *$ & $0.15 * *$ & $0.22 * *$ \\
\hline $\log (K)$ & $0.25 *$ & 0.30 & $0.31 * *$ & $0.10 * *$ & 0.04 & $0.10 * *$ & $0.13 * *$ & $0.14 * *$ \\
\hline$\sigma$ & $0.87 * *$ & $1.36 * *$ & $0.59 * *$ & $0.60 * *$ & $0.67 * *$ & $0.52 * *$ & $0.69 * *$ & $0.63 * *$ \\
\hline$\sigma_{u} / \sigma_{v}$ & 444.46 & 541.50 & 0.83 & $0.91 *$ & $2.00 * *$ & $0.87 * *$ & $1.71 * *$ & $0.99 * *$ \\
\hline short-run ret. to scale & $0.33 * *$ & 0.87 & $0.67 * *$ & $0.49 * *$ & $0.19 * *$ & $0.27 * *$ & $0.28 * *$ & $0.36 * *$ \\
\hline long-run ret. to scale & 0.89 & 1.02 & 0.93 & $1.17 *$ & 0.92 & 1.06 & 1.12 & 1.01 \\
\hline Nob & 45 & 32 & 105 & 316 & 289 & 441 & 491 & 558 \\
\hline Mean of dep.var & 5.42 & 5.53 & 4.14 & 3.35 & 3.75 & 3.60 & 3.45 & 3.34 \\
\hline S.dev of dep.var & 1.80 & 2.00 & 1.79 & 1.49 & 1.61 & 1.62 & 1.67 & 1.70 \\
\hline $\mathrm{R}^{2}$ & 0.86 & 0.79 & 0.92 & 0.88 & 0.91 & 0.92 & 0.91 & 0.90 \\
\hline Mean inefficiency (\%) & -8.21 & -14.56 & -7.24 & -9.58 & -12.09 & -7.54 & -13.23 & -10.48 \\
\hline \multicolumn{9}{|l|}{ Market share equation } \\
\hline Constant & -0.01 & 0.00 & 0.06 & 0.01 & $0.01 *$ & 0.04 & 0.01 & $0.01 * *$ \\
\hline market $\operatorname{share}_{t-1}$ & $0.98 * *$ & $1.03 * *$ & $0.94 * *$ & $0.84 * *$ & $0.79 * *$ & $0.96 * *$ & $0.76 * *$ & $0.94 * *$ \\
\hline efficiency & 0.23 & 2.34 & 15.38 & $3.12 *$ & $3.56 * *$ & 13.55 & $2.29 *$ & $3.06 * *$ \\
\hline import penetration & & 0.00 & 0.00 & 17.63 & -19.19 & 8.23 & 0.00 & 0.00 \\
\hline concentration & 0.07 & 0.04 & 0.91 & $0.51 * *$ & 0.50 & 0.02 & $1.08 *$ & $0.17 *$ \\
\hline Mean of dep.var & 0.20 & 0.22 & 0.10 & 0.09 & 0.08 & 0.08 & 0.06 & 0.06 \\
\hline S.dev of dep.var & 0.31 & 0.31 & 0.25 & 0.35 & 0.31 & 0.34 & 0.28 & 0.24 \\
\hline SEE & 0.04 & 0.05 & 0.15 & 0.05 & 0.07 & 0.20 & 0.08 & 0.03 \\
\hline $\mathrm{R}^{2}$ & 0.99 & 0.98 & 0.65 & 0.98 & 0.95 & 0.65 & 0.92 & 0.98 \\
\hline White-hetero & $27 * *$ & $31 * *$ & $37 * *$ & $102 * *$ & $239 * *$ & $84 * *$ & $460 * *$ & $97 * *$ \\
\hline Reset $\mathrm{y}^{2}$ & 1.34 & 1.87 & $12.83 * *$ & $8.44 * *$ & $41.50 * *$ & $14.30 * *$ & $7.43 * *$ & $16.40 * *$ \\
\hline Reset $\mathrm{y}^{2}, \mathrm{y}^{3}$ & 1.19 & 2.08 & $7.07 * *$ & $19.34 * *$ & $161.98 * *$ & $10.30 * *$ & $178.06 * *$ & $25.29 * *$ \\
\hline \multicolumn{9}{|l|}{ Corr of rel. efficiency } \\
\hline profit margin & & 0.64 & 0.59 & 0.35 & 0.51 & 0.50 & 0.29 & 0.15 \\
\hline investment rate & 0.19 & -0.16 & 0.18 & & & 0.22 & & 0.19 \\
\hline
\end{tabular}

Table 20: Two-equation models: ownership: private

\begin{tabular}{|c|c|c|c|c|c|}
\hline Variable & 1993 & 1994 & 1995 & 1996 & 1997 \\
\hline \multicolumn{6}{|l|}{ Production function } \\
\hline Constant & $0.34 * *$ & $0.34 * *$ & $0.25 * *$ & $0.22 * *$ & $0.23 * *$ \\
\hline $\mathrm{y}_{t-1}$ & $0.59 * *$ & $0.64 * *$ & $0.61 * *$ & $0.76 * *$ & $0.69 * *$ \\
\hline $\log (\mathrm{L})$ & $0.31 * *$ & $0.26 * *$ & $0.27 * *$ & $0.20 * *$ & $0.23 * *$ \\
\hline $\log (K)$ & $0.11 * *$ & $0.08 * *$ & $0.12 * *$ & $0.06 * *$ & $0.09 * *$ \\
\hline$\sigma$ & $0.81 * *$ & $0.70 * *$ & $0.71 * *$ & $0.77 * *$ & $0.71 * *$ \\
\hline$\sigma_{u} / \sigma_{v}$ & $1.29 * *$ & $1.42 * *$ & $1.07 * *$ & $1.69 * *$ & $1.50 * *$ \\
\hline $\begin{array}{l}\text { short-run ret. to scale } \\
\text { long-run ret. to scale }\end{array}$ & $\begin{array}{l}0.42 * * \\
1.02\end{array}$ & $\begin{array}{l}0.35 * * \\
0.97\end{array}$ & $\begin{array}{l}0.39 * * \\
1.00\end{array}$ & $\begin{array}{l}0.26 * * \\
1.08\end{array}$ & $\begin{array}{l}0.32 * * \\
1.04\end{array}$ \\
\hline Nob & 1014 & 1081 & 1591 & 1699 & 1825 \\
\hline Mean of dep.var & 3.54 & 3.88 & 3.72 & 3.54 & 3.46 \\
\hline S.dev of dep.var & 1.32 & 1.33 & 1.42 & 1.50 & 1.53 \\
\hline $\mathrm{R}^{2}$ & 0.77 & 0.84 & 0.83 & 0.85 & 0.88 \\
\hline Mean inefficiency (\%) & -14.28 & -11.53 & -11.09 & -14.57 & -13.17 \\
\hline \multicolumn{6}{|l|}{ Market share equation } \\
\hline Constant & $0.01 * *$ & $0.01 *$ & $0.01 * *$ & $0.01 * *$ & $0.00 * *$ \\
\hline market share $_{t-1}$ & $0.86 * *$ & $0.81 * *$ & $0.81 * *$ & $0.94 * *$ & $0.91 * *$ \\
\hline efficiency & $1.11 * *$ & $2.35 *$ & $2.15 * *$ & $1.09 * *$ & $0.87 * *$ \\
\hline import penetration & $0.00 * *$ & 0.00 & 0.00 & 0.00 & $0.00 * *$ \\
\hline concentration & $0.37 *$ & 0.17 & -0.07 & 0.13 & $0.17 * *$ \\
\hline Mean of dep.var & 0.03 & 0.03 & 0.03 & 0.03 & 0.03 \\
\hline S.dev of dep.var & 0.09 & 0.09 & 0.09 & 0.09 & 0.07 \\
\hline SEE & 0.03 & 0.04 & 0.03 & 0.03 & 0.02 \\
\hline $\mathrm{R}^{2}$ & 0.92 & 0.83 & 0.86 & 0.88 & 0.92 \\
\hline White-hetero & $283 * *$ & $178 * *$ & $740 * *$ & $467 * *$ & $471 * *$ \\
\hline Reset $y^{2}$ & 0.83 & 1.75 & $74.85 * *$ & $10.63 * *$ & $4.78 *$ \\
\hline Reset $\mathrm{y}^{2}, \mathrm{y}^{3}$ & $17.08 * *$ & $17.31 * *$ & $80.14 * *$ & $18.73 * *$ & $7.40 * *$ \\
\hline \multicolumn{6}{|l|}{ Corr of rel. efficiency } \\
\hline $\begin{array}{l}\text { profit margin } \\
\text { investment rate }\end{array}$ & 0.50 & 0.38 & 0.40 & 0.27 & 0.25 \\
\hline
\end{tabular}


Table 21: Two-equation models: ownership: state

\begin{tabular}{|c|c|c|c|c|c|c|}
\hline Variable & 1992 & 1993 & 1994 & 1995 & 1996 & 1997 \\
\hline \multicolumn{7}{|l|}{ Production function } \\
\hline Constant & -0.09 & -0.02 & 0.04 & $-0.17 *$ & 0.11 & -0.10 \\
\hline $\mathrm{y}_{t-1}$ & $0.19 * *$ & $0.41 * *$ & $0.59 * *$ & $0.60 * *$ & $0.67 * *$ & $0.58 * *$ \\
\hline $\log (\mathrm{L})$ & $0.67 * *$ & $0.51 * *$ & $0.35 * *$ & $0.38 * *$ & $0.26 * *$ & $0.38 * *$ \\
\hline $\log (\mathrm{K})$ & $0.16 * *$ & $0.09 * *$ & $0.08 * *$ & $0.07 * *$ & $0.07 * *$ & $0.07 * *$ \\
\hline$\sigma$ & $0.94 * *$ & $0.89 * *$ & $0.69 * *$ & $0.73 * *$ & $0.68 * *$ & $0.65 * *$ \\
\hline$\sigma_{u} / \sigma_{v}$ & $2.10 * *$ & $1.78 * *$ & $1.95 * *$ & $1.79 * *$ & $1.86 * *$ & $1.21 * *$ \\
\hline $\begin{array}{l}\text { short-run ret. to scale } \\
\text { long-run ret. to scale }\end{array}$ & $0.83 * *$ & $0.60 * *$ & $0.43 * *$ & $0.45 * *$ & $0.33 * *$ & $0.45 * *$ \\
\hline Nob & 788 & 1072 & 817 & 848 & 693 & 638 \\
\hline Mean of dep.var & 4.27 & 3.89 & 4.31 & 4.02 & 3.89 & 3.79 \\
\hline S.dev of dep.var & 1.60 & 1.60 & 1.63 & 1.68 & 1.69 & 1.63 \\
\hline $\mathrm{R}^{2}$ & 0.83 & 0.83 & 0.90 & 0.90 & 0.91 & 0.90 \\
\hline Mean inefficiency (\%) & -15.56 & -15.31 & -10.81 & -12.12 & -11.73 & -10.26 \\
\hline \multicolumn{7}{|l|}{ Market share equation } \\
\hline Constant & $0.02 * *$ & $0.01 *$ & $0.01 *$ & $0.01 * *$ & 0.00 & 0.00 \\
\hline market share $t_{t-1}$ & $0.49 * *$ & $0.86 * *$ & $0.81 * *$ & $0.92 * *$ & $1.03 * *$ & $0.96 * *$ \\
\hline efficiency & $1.46 * *$ & $1.19 * *$ & $1.92 * *$ & $0.92 * *$ & 0.71 & $1.09 *$ \\
\hline import penetration & $-0.03 * *$ & 0.00 & $-0.01 * *$ & 0.00 & -0.01 & 0.00 \\
\hline concentration & $1.74 * *$ & 0.36 & $0.34 *$ & 0.05 & 0.14 & $0.07 *$ \\
\hline Mean of dep.var & 0.05 & 0.05 & 0.05 & 0.05 & 0.05 & 0.04 \\
\hline S.dev of dep.var & 0.13 & 0.19 & 0.20 & 0.18 & 0.20 & 0.20 \\
\hline SEE & 0.08 & 0.03 & 0.05 & 0.03 & 0.04 & 0.02 \\
\hline $\mathrm{R}^{2}$ & 0.61 & 0.97 & 0.95 & 0.98 & 0.95 & 0.99 \\
\hline White-hetero & $237 * *$ & $566 * *$ & $646 * *$ & $37 * *$ & $158 * *$ & $182 * *$ \\
\hline Reset $\mathrm{y}^{2}$ & $32.89 * *$ & $16.65 * *$ & $42.65 * *$ & $69.31 * *$ & $171.94 * *$ & $39.02 * *$ \\
\hline Reset $\mathrm{y}^{2}, \mathrm{y}^{3}$ & $25.09 * *$ & $62.34 * *$ & $193.86 * *$ & $36.00 * *$ & $85.85 * *$ & $19.62 * *$ \\
\hline \multicolumn{7}{|l|}{ Corr of rel. efficiency } \\
\hline $\begin{array}{l}\text { profit margin } \\
\text { investment rate }\end{array}$ & 0.24 & 0.43 & 0.43 & 0.23 & & 0.15 \\
\hline
\end{tabular}

Table 22: Two-equation models: ownership: foreign majority

\begin{tabular}{|c|c|c|c|c|c|c|}
\hline Variable & 1992 & 1993 & 1994 & 1995 & 1996 & 1997 \\
\hline \multicolumn{7}{|l|}{ Production function } \\
\hline Constant & 0.38 & $0.84 * *$ & $0.61 * *$ & $0.53 * *$ & $0.36 * *$ & $0.27 * *$ \\
\hline $\mathrm{y}_{t-1}$ & $0.17 * *$ & $0.51 * *$ & $0.65 * *$ & $0.67 * *$ & $0.64 * *$ & $0.72 * *$ \\
\hline $\log (\mathrm{L})$ & $0.45 * *$ & $0.25 * *$ & $0.21 * *$ & $0.17 * *$ & $0.23 * *$ & $0.20 * *$ \\
\hline $\log (\mathrm{K})$ & $0.30 * *$ & $0.19 * *$ & $0.13 * *$ & $0.15 * *$ & $0.15 * *$ & $0.11 * *$ \\
\hline$\sigma$ & 0.55 & $0.86 * *$ & $0.67 * *$ & $0.75 * *$ & $0.70 * *$ & $0.65 * *$ \\
\hline$\sigma_{u} / \sigma_{v}$ & 0.05 & $1.34 * *$ & $1.36 * *$ & $1.22 * *$ & $1.02 * *$ & $1.02 * *$ \\
\hline $\begin{array}{l}\text { short-run ret. to scale } \\
\text { long-run ret. to scale }\end{array}$ & $\begin{array}{l}0.75 * * \\
0.90\end{array}$ & $\begin{array}{l}0.43 * * \\
0.88 *\end{array}$ & $\begin{array}{l}0.33 * * \\
0.96\end{array}$ & $\begin{array}{l}0.32 * * \\
0.95\end{array}$ & $\begin{array}{l}0.38 * * \\
1.04\end{array}$ & $\begin{array}{l}0.31 * * \\
1.13 * *\end{array}$ \\
\hline Nob & 174 & 614 & 603 & 1021 & 1201 & 1387 \\
\hline Mean of dep.var & 4.61 & 4.39 & 4.68 & 4.35 & 4.33 & 4.31 \\
\hline S.dev of dep.var & 1.24 & 1.48 & 1.45 & 1.55 & 1.57 & 1.64 \\
\hline $\mathrm{R}^{2}$ & 0.80 & 0.80 & 0.87 & 0.85 & 0.86 & 0.89 \\
\hline Mean inefficiency (\%) & -0.48 & -12.21 & -8.94 & -10.45 & -9.07 & -8.51 \\
\hline \multicolumn{7}{|l|}{ Market share equation } \\
\hline Constant & 0.27 & $0.01 * *$ & $0.02 * *$ & $0.02 * *$ & 0.05 & $0.04 * *$ \\
\hline market $\operatorname{share}_{t-1}$ & $0.47 *$ & $0.94 * *$ & $1.12 * *$ & $1.15 * *$ & $0.65 * *$ & $0.23 * *$ \\
\hline efficiency & 1169.83 & $1.98 * *$ & $3.83 * *$ & $4.45 *$ & 9.48 & $4.37 * *$ \\
\hline import penetration & $-0.02 * *$ & 0.00 & 0.00 & -0.01 & $-0.02 * *$ & $-0.02 * *$ \\
\hline concentration & $2.90 * *$ & 0.18 & -0.19 & 0.21 & $1.74 * *$ & $2.03 * *$ \\
\hline Mean of dep.var & 0.06 & 0.05 & 0.06 & 0.06 & 0.06 & 0.05 \\
\hline S.dev of dep.var & 0.12 & 0.14 & 0.17 & 0.21 & 0.28 & 0.15 \\
\hline SEE & 0.07 & 0.04 & 0.05 & 0.13 & 0.24 & 0.11 \\
\hline $\mathrm{R}^{2}$ & 0.64 & 0.91 & 0.93 & 0.62 & 0.26 & 0.45 \\
\hline White-hetero & $56 * *$ & $254 * *$ & $82 * *$ & $143 * *$ & $43 * *$ & $960 * *$ \\
\hline Reset $\mathrm{y}^{2}$ & $4.89 *$ & $83.62 * *$ & $20.41 * *$ & 0.98 & $5.38 *$ & $4961.67 * *$ \\
\hline Reset $\mathrm{y}^{2}, \mathrm{y}^{3}$ & 2.43 & $78.57 * *$ & $10.25 * *$ & 0.85 & $3.67 *$ & $2488.91 * *$ \\
\hline \multicolumn{7}{|l|}{ Corr of rel. efficiency } \\
\hline $\begin{array}{l}\text { profit margin } \\
\text { investment rate }\end{array}$ & 0.46 & 0.25 & & 0.40 & 0.44 & 0.19 \\
\hline
\end{tabular}


Table 23: Two-equation models: ownership: important foreign

\begin{tabular}{|c|c|c|c|c|c|c|}
\hline Variable & 1992 & 1993 & 1994 & 1995 & 1996 & 1997 \\
\hline \multicolumn{7}{|l|}{ Production function } \\
\hline Constant & 0.47 & $0.58 * *$ & $0.57 * *$ & $0.32 *$ & 0.22 & -0.19 \\
\hline $\mathrm{y}_{t-1}$ & $0.17 *$ & $0.52 * *$ & $0.69 * *$ & $0.67 * *$ & $0.77 * *$ & $0.67 * *$ \\
\hline $\log (\mathrm{L})$ & $0.55 * *$ & $0.30 * *$ & $0.16 * *$ & $0.16 * *$ & $0.14 * *$ & $0.20 * *$ \\
\hline $\log (\mathrm{K})$ & $0.25 * *$ & $0.16 * *$ & $0.13 * *$ & $0.16 * *$ & $0.12 * *$ & $0.17 * *$ \\
\hline$\sigma$ & $0.94 * *$ & $0.80 * *$ & $0.73 * *$ & $0.60 * *$ & $0.65 * *$ & 0.50 \\
\hline$\sigma_{u} / \sigma_{v}$ & $1.42 *$ & $1.70 * *$ & $1.44 * *$ & $0.92 * *$ & $1.28 * *$ & 0.03 \\
\hline short-run ret. to scale & $0.81 *$ & $0.46 * *$ & $0.28 * *$ & $0.32 * *$ & $0.25 * *$ & $0.37 * *$ \\
\hline long-run ret. to scale & 0.97 & 0.96 & 0.90 & 0.98 & 1.12 & 1.11 \\
\hline Nob & 100 & 269 & 229 & 315 & 325 & 350 \\
\hline Mean of dep.var & 3.83 & 4.08 & 4.37 & 4.14 & 3.98 & 3.92 \\
\hline S.dev of dep.var & 1.41 & 1.52 & 1.54 & 1.71 & 1.82 & 1.75 \\
\hline $\mathrm{R}^{2}$ & 0.74 & 0.85 & 0.87 & 0.91 & 0.92 & 0.91 \\
\hline Mean inefficiency (\%) & -15.89 & -13.23 & -10.67 & -7.74 & -10.11 & -0.34 \\
\hline \multicolumn{7}{|l|}{ Market share equation } \\
\hline Constant & $0.01 * *$ & 0.00 & 0.01 & $0.02 * *$ & 0.00 & $0.39 * *$ \\
\hline market $\operatorname{share}_{t-1}$ & $0.52 * *$ & -0.07 & $0.66 * *$ & $0.86 * *$ & $1.09 * *$ & $0.97 * *$ \\
\hline efficiency & $1.07 * *$ & 0.34 & $2.35 * *$ & $3.63 *$ & 0.30 & $2939.77 * *$ \\
\hline import penetration & $0.00 * *$ & -0.04 & -0.01 & $0.00 *$ & 0.00 & 0.00 \\
\hline concentration & $0.80 * *$ & 5.43 & 0.80 & 0.03 & -0.02 & $0.20 * *$ \\
\hline Mean of dep.var & 0.02 & 0.07 & 0.07 & 0.06 & 0.05 & 0.05 \\
\hline S.dev of dep.var & 0.04 & 0.39 & 0.32 & 0.25 & 0.26 & 0.25 \\
\hline SEE & 0.02 & 0.18 & 0.10 & 0.04 & 0.04 & 0.03 \\
\hline $\mathrm{R}^{2}$ & 0.79 & 0.78 & 0.91 & 0.98 & 0.98 & 0.99 \\
\hline White-hetero & $35 * *$ & $267 * *$ & $227 * *$ & $236 * *$ & $138 * *$ & $216 * *$ \\
\hline Reset $\mathrm{y}^{2}$ & $7.34 * *$ & $10.27 * *$ & 3.52 & $10.63 * *$ & $93.37 * *$ & 0.00 \\
\hline Reset $\mathrm{y}^{2}, \mathrm{y}^{3}$ & $5.09 * *$ & $883.52 * *$ & $802.15 * *$ & $169.44 * *$ & $47.10 * *$ & 0.13 \\
\hline \multicolumn{7}{|l|}{ Corr of rel. efficiency } \\
\hline profit margin & 0.57 & 0.26 & 0.36 & 0.49 & 0.44 & 0.29 \\
\hline investment rate & 0.29 & & 0.38 & 0.61 & & 0.18 \\
\hline
\end{tabular}

Table 24: Two-equation models: ownership: other

\begin{tabular}{|c|c|c|c|c|c|c|}
\hline Variable & 1992 & 1993 & 1994 & 1995 & 1996 & 1997 \\
\hline \multicolumn{7}{|l|}{ Production function } \\
\hline Constant & 0.02 & $0.14 * *$ & $0.15 * *$ & 0.07 & $0.15 * *$ & $0.24 * *$ \\
\hline $\mathrm{y}_{t-1}$ & $0.25 * *$ & $0.50 * *$ & $0.66 * *$ & $0.67 * *$ & $0.73 * *$ & $0.71 * *$ \\
\hline $\log (\mathrm{L})$ & $0.65 * *$ & $0.43 * *$ & $0.29 * *$ & $0.28 * *$ & $0.22 * *$ & $0.21 * *$ \\
\hline $\log (\mathrm{K})$ & $0.04 * *$ & $0.02 * *$ & $0.05 * *$ & $0.06 * *$ & $0.05 * *$ & $0.07 * *$ \\
\hline$\sigma$ & $0.81 * *$ & $0.81 * *$ & $0.65 * *$ & $0.69 * *$ & $0.69 * *$ & $0.71 * *$ \\
\hline$\sigma_{u} / \sigma_{v}$ & $1.50 * *$ & $1.29 * *$ & $1.83 * *$ & $1.58 * *$ & $1.55 * *$ & $1.65 * *$ \\
\hline short-run ret. to scale & $0.69 * *$ & $0.45 * *$ & $0.34 * *$ & $0.34 * *$ & $0.27 * *$ & $0.27 * *$ \\
\hline long-run ret. to scale & $0.91 * *$ & $0.90 * *$ & 0.98 & 1.02 & 0.99 & $0.93 * *$ \\
\hline Nob & 2394 & 3421 & 3062 & 4382 & 4905 & 5697 \\
\hline Mean of dep.var & 3.34 & 3.05 & 3.17 & 2.83 & 2.70 & 2.53 \\
\hline S.dev of dep.var & 1.05 & 1.09 & 1.05 & 1.13 & 1.17 & 1.20 \\
\hline $\mathrm{R}^{2}$ & 0.66 & 0.66 & 0.79 & 0.79 & 0.80 & 0.80 \\
\hline Mean inefficiency (\%) & -15.90 & -16.49 & -13.94 & -16.09 & -16.79 & -18.60 \\
\hline \multicolumn{7}{|l|}{ Market share equation } \\
\hline Constant & $0.01 * *$ & 0.00 & $0.00 * *$ & 0.00 & $0.00 * *$ & $0.00 * *$ \\
\hline market $\operatorname{share}_{t-1}$ & $0.66 * *$ & $0.73 * *$ & $0.74 * *$ & $0.97 * *$ & $0.81 * *$ & $0.80 * *$ \\
\hline efficiency & $0.98 * *$ & 0.23 & $0.35 * *$ & $0.26 * *$ & $0.55 * *$ & $0.27 * *$ \\
\hline import penetration & $-0.01 * *$ & 0.00 & 0.00 & 0.00 & 0.00 & $0.00 *$ \\
\hline concentration & $0.77 * *$ & 0.22 & 0.21 & 0.27 & 0.27 & 0.20 \\
\hline Mean of dep.var & 0.01 & 0.01 & 0.01 & 0.01 & 0.01 & 0.01 \\
\hline S.dev of dep.var & 0.04 & 0.03 & 0.03 & 0.03 & 0.03 & 0.02 \\
\hline SEE & 0.03 & 0.02 & 0.01 & 0.01 & 0.01 & 0.01 \\
\hline $\mathrm{R}^{2}$ & 0.43 & 0.78 & 0.73 & 0.80 & 0.83 & 0.86 \\
\hline White-hetero & $480 * *$ & $1575 * *$ & $708 * *$ & $1199 * *$ & $1349 * *$ & $1806 * *$ \\
\hline Reset $\mathrm{y}^{2}$ & $5.11 *$ & 0.01 & 0.00 & $217.24 * *$ & $32.89 * *$ & 1.45 \\
\hline Reset $\mathrm{y}^{2}, \mathrm{y}^{3}$ & $66.04 * *$ & 2.88 & $364.21 * *$ & $110.83 * *$ & $319.78 * *$ & $96.59 * *$ \\
\hline \multicolumn{7}{|l|}{ Corr of rel. efficiency } \\
\hline $\begin{array}{l}\text { profit margin } \\
\text { investment rate }\end{array}$ & 0.51 & 0.16 & 0.31 & 0.16 & 0.20 & $\begin{array}{l}0.21 \\
0.20\end{array}$ \\
\hline
\end{tabular}


Table 25: Two-equation models: small firms

\begin{tabular}{|c|c|c|c|c|c|c|c|c|}
\hline Variable & 1990 & 1991 & 1992 & 1993 & 1994 & 1995 & 1996 & 1997 \\
\hline \multicolumn{9}{|l|}{ Production function } \\
\hline Constant & 0.35 & $0.71 * *$ & $0.32 * *$ & $0.15 *$ & 0.10 & 0.01 & $0.08 *$ & $0.15 * *$ \\
\hline $\mathrm{y}_{t-1}$ & $0.71 * *$ & $0.78 * *$ & $0.19 * *$ & $0.57 * *$ & $0.69 * *$ & $0.69 * *$ & $0.75 * *$ & $0.72 * *$ \\
\hline $\log (\mathrm{L})$ & $0.20 * *$ & 0.04 & $0.62 * *$ & $0.38 * *$ & $0.29 * *$ & $0.28 * *$ & $0.24 * *$ & $0.23 * *$ \\
\hline $\log (K)$ & 0.01 & -0.03 & $0.06 * *$ & $0.08 * *$ & $0.06 * *$ & $0.09 * *$ & $0.07 * *$ & $0.08 * *$ \\
\hline$\sigma$ & $0.90 * *$ & $0.89 * *$ & $0.90 * *$ & $0.87 * *$ & $0.72 * *$ & $0.76 * *$ & $0.78 * *$ & $0.74 * *$ \\
\hline$\sigma_{u} / \sigma_{v}$ & $1.45 * *$ & $1.93 * *$ & $1.42 * *$ & $1.33 * *$ & $1.57 * *$ & $1.38 * *$ & $1.60 * *$ & $1.50 * *$ \\
\hline short-run ret. to scale & $0.21 * *$ & $0.01 * *$ & $0.68 * *$ & $0.45 * *$ & $0.35 * *$ & $0.37 * *$ & $0.31 * *$ & $0.31 * *$ \\
\hline long-run ret. to scale & 0.73 & $0.05 * *$ & $0.84 * *$ & 1.06 & $1.13 *$ & $1.21 * *$ & $1.24 * *$ & $1.12 * *$ \\
\hline Nob & 569 & 370 & 1441 & 3423 & 2821 & 4917 & 5653 & 6610 \\
\hline Mean of dep.var & 3.19 & 2.85 & 2.75 & 2.71 & 2.79 & 2.59 & 2.48 & 2.36 \\
\hline S.dev of dep.var & 0.97 & 0.94 & 0.87 & 0.99 & 0.94 & 1.04 & 1.10 & 1.12 \\
\hline $\mathrm{R}^{2}$ & 0.49 & 0.53 & 0.39 & 0.53 & 0.66 & 0.68 & 0.72 & 0.74 \\
\hline Mean inefficiency (\%) & -18.07 & -21.77 & -21.22 & -20.28 & -17.08 & -18.65 & -20.65 & -20.36 \\
\hline \multicolumn{9}{|l|}{ Market share equation } \\
\hline Constant & $0.00 * *$ & $0.00 * *$ & $0.01 * *$ & 0.00 & $0.01 * *$ & $0.00 * *$ & $0.00 * *$ & $0.00 *$ \\
\hline market share ${ }_{t-1}$ & 0.10 & $0.66 * *$ & 0.14 & $0.54 *$ & $0.46 * *$ & $0.77 * *$ & $0.71 * *$ & $0.99 * *$ \\
\hline efficiency & $0.20 * *$ & $0.14 * *$ & $0.79 * *$ & $0.54 * *$ & $0.60 * *$ & $0.54 * *$ & $0.51 * *$ & $0.34 * *$ \\
\hline import penetration & $-0.03 *$ & 0.00 & $-0.01 *$ & -0.02 & $0.00 * *$ & 0.00 & $-0.01 * *$ & 0.00 \\
\hline concentration & $0.25 *$ & $0.08 *$ & $0.96 * *$ & 1.64 & $0.31 * *$ & $0.18 *$ & $0.44 *$ & -0.02 \\
\hline Mean of dep.var & 0.01 & 0.00 & 0.01 & 0.01 & 0.01 & 0.01 & 0.01 & 0.01 \\
\hline S.dev of dep.var & 0.01 & 0.01 & 0.03 & 0.08 & 0.04 & 0.04 & 0.04 & 0.03 \\
\hline SEE & 0.01 & 0.00 & 0.02 & 0.06 & 0.02 & 0.02 & 0.02 & 0.01 \\
\hline $\mathrm{R}^{2}$ & 0.29 & 0.85 & 0.46 & 0.32 & 0.85 & 0.87 & 0.80 & 0.87 \\
\hline White-hetero & $313 * *$ & $88 * *$ & $623 * *$ & $841 * *$ & $473 * *$ & $1930 * *$ & $3761 * *$ & $4566 * *$ \\
\hline Reset $\mathrm{y}^{2}$ & $409.71 * *$ & 1.49 & 0.26 & $9.07 * *$ & $595.51 * *$ & $798.50 * *$ & $1368.32 * *$ & $2175.52 * *$ \\
\hline Reset $\mathrm{y}^{2}, \mathrm{y}^{3}$ & $209.58 * *$ & $8.93 * *$ & $63.10 * *$ & $334.40 * *$ & $349.12 * *$ & $495.04 * *$ & $817.39 * *$ & $1198.71 * *$ \\
\hline \multicolumn{9}{|l|}{ Corr of rel. efficiency } \\
\hline $\begin{array}{l}\text { profit margin } \\
\text { investment rate }\end{array}$ & 0.36 & 0.58 & 0.31 & 0.17 & 0.21 & & 0.16 & \\
\hline
\end{tabular}

Table 26: Two-equation models: medium-sized firms

\begin{tabular}{|c|c|c|c|c|c|c|c|c|}
\hline Variable & 1990 & 1991 & 1992 & 1993 & 1994 & 1995 & 1996 & 1997 \\
\hline \multicolumn{9}{|l|}{ Production function } \\
\hline Constant & $0.56 * *$ & 0.16 & -0.25 & $0.41 * *$ & $0.25 * *$ & 0.12 & $0.40 * *$ & 0.11 \\
\hline $\mathrm{y}_{t-1}$ & $0.63 * *$ & $0.47 * *$ & $0.22 * *$ & $0.45 * *$ & $0.62 * *$ & $0.65 * *$ & $0.74 * *$ & $0.71 * *$ \\
\hline $\log (\mathrm{L})$ & $0.09 * *$ & $0.31 * *$ & $0.55 * *$ & $0.35 * *$ & $0.23 * *$ & $0.23 * *$ & $0.09 * *$ & $0.16 * *$ \\
\hline $\log (\mathrm{K})$ & $0.17 * *$ & $0.11 * *$ & $0.24 * *$ & $0.13 * *$ & $0.14 * *$ & $0.12 * *$ & $0.13 * *$ & $0.15 * *$ \\
\hline$\sigma$ & $0.73 * *$ & $0.86 * *$ & $0.82 * *$ & $0.87 * *$ & $0.62 * *$ & $0.64 * *$ & $0.62 * *$ & $0.61 * *$ \\
\hline$\sigma_{u} / \sigma_{v}$ & $1.94 * *$ & $2.10 * *$ & $1.62 * *$ & $1.61 * *$ & $1.58 * *$ & $1.39 * *$ & $1.56 * *$ & $1.20 * *$ \\
\hline short-run ret. to scale & $0.27 * *$ & $0.42 * *$ & $0.79 * *$ & $0.49 * *$ & $0.37 * *$ & $0.36 * *$ & $0.22 * *$ & $0.31 * *$ \\
\hline long-run ret. to scale & $0.73 * *$ & $0.80 * *$ & 1.01 & $0.88 * *$ & 0.97 & 1.02 & $0.85 *$ & 1.07 \\
\hline Nob & 894 & 633 & 1659 & 2419 & 2350 & 2567 & 2550 & 2614 \\
\hline Mean of dep.var & 3.91 & 3.56 & 3.85 & 3.89 & 4.06 & 4.09 & 4.09 & 4.06 \\
\hline S.dev of dep.var & 0.78 & 0.81 & 0.84 & 0.91 & 0.85 & 0.87 & 0.88 & 0.91 \\
\hline $\mathrm{R}^{2}$ & 0.54 & 0.42 & 0.47 & 0.49 & 0.69 & 0.68 & 0.71 & 0.71 \\
\hline Mean inefficiency (\%) & -12.81 & -16.79 & -14.26 & -14.85 & -10.00 & -9.80 & -9.87 & -8.96 \\
\hline \multicolumn{9}{|l|}{ Market share equation } \\
\hline Constant & $0.00 *$ & $0.00 *$ & $0.02 * *$ & $0.00 *$ & $0.01 * *$ & 0.00 & $0.01 * *$ & $0.01 * *$ \\
\hline market share $_{t-1}$ & $0.64 * *$ & $0.46 * *$ & 0.13 & $0.80 * *$ & $0.79 * *$ & $1.24 * *$ & $0.61 * *$ & $0.88 * *$ \\
\hline efficiency & $0.42 * *$ & 0.00 & $1.56 * *$ & 0.52 & $1.96 * *$ & $1.52 * *$ & $1.70 * *$ & $1.60 * *$ \\
\hline import penetration & -0.02 & $-0.06 * *$ & $-0.01 * *$ & 0.00 & $0.00 *$ & 0.00 & $-0.01 *$ & $0.00 * *$ \\
\hline concentration & 0.20 & $0.95 * *$ & $1.61 * *$ & 0.24 & 0.19 & 0.09 & $1.14 *$ & $0.19 * *$ \\
\hline Mean of dep.var & 0.01 & 0.01 & 0.02 & 0.02 & 0.02 & 0.02 & 0.02 & 0.02 \\
\hline S.dev of dep.var & 0.04 & 0.03 & 0.06 & 0.06 & 0.05 & 0.11 & 0.09 & 0.08 \\
\hline SEE & 0.01 & 0.01 & 0.04 & 0.02 & 0.03 & 0.08 & 0.04 & 0.02 \\
\hline $\mathrm{R}^{2}$ & 0.90 & 0.86 & 0.39 & 0.84 & 0.73 & 0.44 & 0.76 & 0.95 \\
\hline White-hetero & $779 * *$ & $192 * *$ & $483 * *$ & $889 * *$ & $283 * *$ & $482 * *$ & $409 * *$ & $743 * *$ \\
\hline Reset $\mathrm{y}^{2}$ & $8.70 * *$ & $115.40 * *$ & $144.75 * *$ & $10.43 * *$ & $34.26 * *$ & $48.38 * *$ & $184.18 * *$ & 3.65 \\
\hline Reset $\mathrm{y}^{2}, \mathrm{y}^{3}$ & $76.19 * *$ & $77.26 * *$ & $83.92 * *$ & $7.50 * *$ & $17.99 * *$ & $30.92 * *$ & $94.91 * *$ & $4.62 *$ \\
\hline \multicolumn{9}{|l|}{ Corr of rel. efficiency } \\
\hline profit margin & 0.32 & 0.47 & 0.36 & 0.43 & 0.39 & 0.35 & 0.38 & 0.37 \\
\hline investment rate & 0.20 & & & 0.20 & & 0.26 & 0.23 & 0.22 \\
\hline
\end{tabular}


Table 27: Two-equation models: large firms

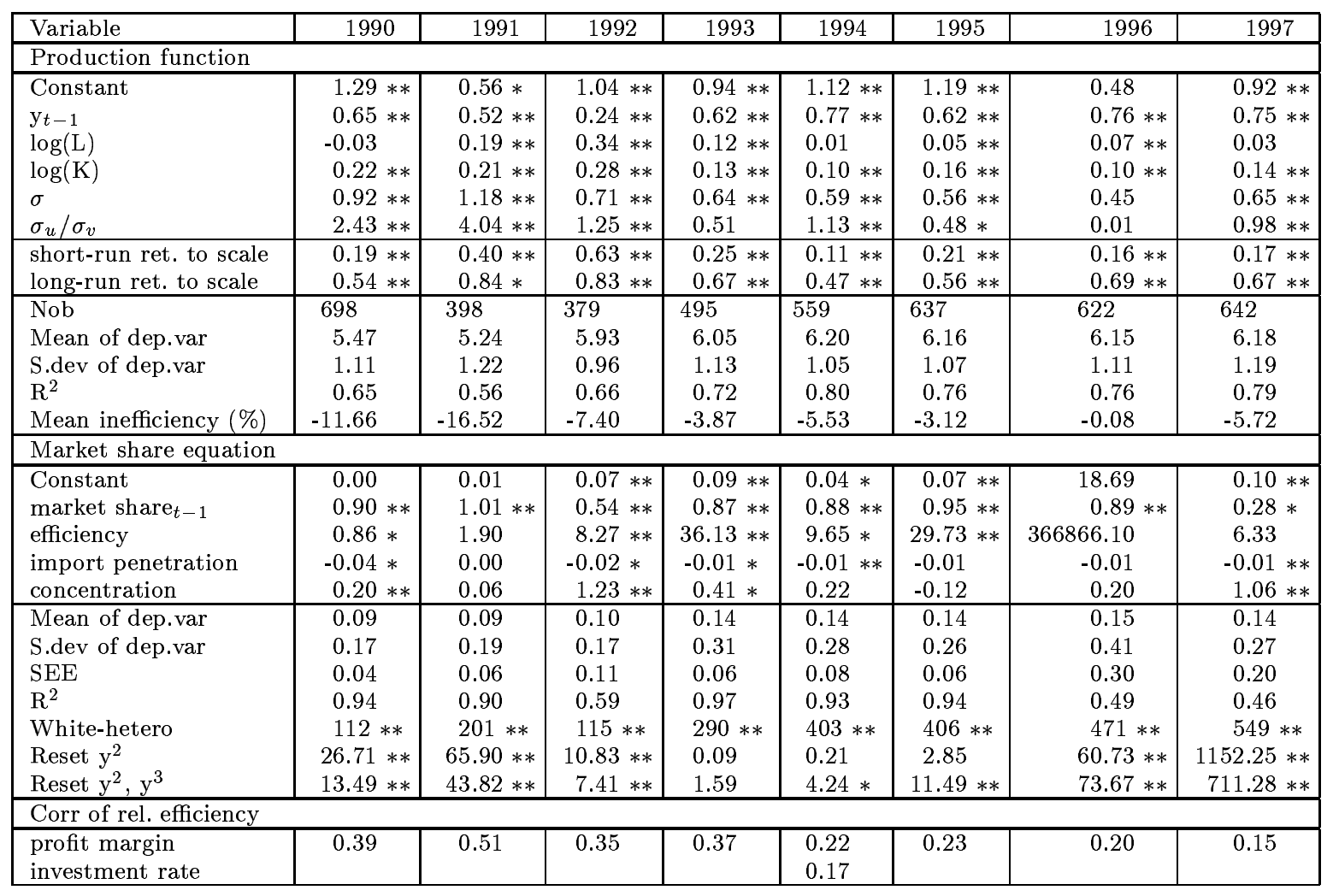




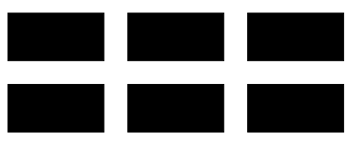

THE WILLIAM DAVIDSON INSTITUTE

AT THE UNIVERSITY OF MICHIGAN BUSINESSSCHOOL

\section{DAVIDSON INSTITUTE WORKING PAPER SERIES}

CURRENT AS OF 8/30/00

\begin{tabular}{|c|c|c|}
\hline Publication & Authors & Date of Paper \\
\hline $\begin{array}{l}\text { No. } 333 \text { Efficiency and Market Share in } \\
\text { Hungarian Corporate Sector }\end{array}$ & László Halpern and Gábor Körösi & July 2000 \\
\hline $\begin{array}{l}\text { No. } 332 \text { Search-Money-and-Barter Models of } \\
\text { Financial Stabilization }\end{array}$ & S.I. Boyarchenko and S.Z. Levendorskii & July 2000 \\
\hline $\begin{array}{l}\text { No. } 331 \text { Worker Training in a Restructuring } \\
\text { Economy: Evidence from the Russian } \\
\text { Transition }\end{array}$ & $\begin{array}{l}\text { Mark C. Berger, John S. Earle and Klara } \\
\text { Z. Sabirianova }\end{array}$ & August 2000 \\
\hline $\begin{array}{l}\text { No. 330 Economic Development in Palanpur } \\
\text { 1957-1993: A Sort of Growth }\end{array}$ & Peter Lanjouw & August 2000 \\
\hline $\begin{array}{l}\text { No. } 329 \text { Trust, Organizational Controls, } \\
\text { Knowledge Acquisition from the Foreign } \\
\text { Parents, and Performance in Vietnamese } \\
\text { International Joint Ventures }\end{array}$ & $\begin{array}{l}\text { Marjorie A. Lyles, Le Dang Doanh, and } \\
\text { Jeffrey } Q . \text { Barden }\end{array}$ & June 2000 \\
\hline $\begin{array}{l}\text { No. } 328 \text { Comparative Advertising in the } \\
\text { Global Marketplace: The Effects of Cultural } \\
\text { Orientation on Communication }\end{array}$ & $\begin{array}{l}\text { Zeynep Gürhan-Canli and Durairaj } \\
\text { Maheswaran }\end{array}$ & August 2000 \\
\hline $\begin{array}{l}\text { No. } 327 \text { Post Privatization Enterprise } \\
\text { Restructuring }\end{array}$ & Morris Bornstein & July 2000 \\
\hline No. 326 Who is Afraid of Political Instability? & Nauro F. Campos and Jeffrey B. Nugent & July 2000 \\
\hline $\begin{array}{l}\text { No. } 325 \text { Business Groups, the Financial } \\
\text { Market and Modernization }\end{array}$ & Raja Kali & June 2000 \\
\hline $\begin{array}{l}\text { No. } 324 \text { Restructuring with What Success? A } \\
\text { Case Study of Russian Firms }\end{array}$ & Susan Linz & July 2000 \\
\hline $\begin{array}{l}\text { No. } 323 \text { Priorities and Sequencing in } \\
\text { Privatization: Theory and Evidence from the } \\
\text { Czech Republic }\end{array}$ & $\begin{array}{l}\text { Nandini Gupta, John C. Ham and Jan } \\
\text { Svejnar }\end{array}$ & May 2000 \\
\hline $\begin{array}{l}\text { No. } 322 \text { Liquidity, Volatility, and Equity } \\
\text { Trading Costs Across Countries and Over } \\
\text { Time }\end{array}$ & $\begin{array}{l}\text { Ian Domowitz, Jack Glen and Ananth } \\
\text { Madhavan }\end{array}$ & March 2000 \\
\hline $\begin{array}{l}\text { No. } 321 \text { Equilibrium Wage Arrears: } \\
\text { Institutional Lock-In of Contractual Failure in } \\
\text { Russia }\end{array}$ & John S. Earle and Klara Z. Sabirianova & June 2000 \\
\hline $\begin{array}{l}\text { No. } 320 \text { Rethinking Marketing Programs for } \\
\text { Emerging Markets }\end{array}$ & Niraj Dawar and Amitava Chattopadhyay & June 2000 \\
\hline $\begin{array}{l}\text { No. } 319 \text { Public Finance and Low Equilibria in } \\
\text { Transition Economies; the Role of Institutions }\end{array}$ & Daniel Daianu and Radu Vranceanu & June 2000 \\
\hline $\begin{array}{l}\text { No. } 318 \text { Some Econometric Evidence on the } \\
\text { Effectiveness of Active Labour Market } \\
\text { Programmes in East Germany }\end{array}$ & Martin Eichler and Michael Lechner & June 2000 \\
\hline $\begin{array}{l}\text { No. } 317 \text { A Model of Russia's “Virtual } \\
\text { Economy” }\end{array}$ & R.E Ericson and B.W Ickes & May 2000 \\
\hline $\begin{array}{l}\text { No. } 316 \text { Financial Institutions, Financial } \\
\text { Contagion, and Financial Crises }\end{array}$ & Haizhou Huang and Chenggang Xu & March 2000 \\
\hline $\begin{array}{l}\text { No. } 315 \text { Privatization versus Regulation in } \\
\text { Developing Economies: The Case of West } \\
\text { African Banks }\end{array}$ & $\begin{array}{l}\text { Jean Paul Azam, Bruno Biais, and } \\
\text { Magueye Dia }\end{array}$ & February 2000 \\
\hline
\end{tabular}

Publications denoted by an asterisk are not available on the Kresge Library webpage (http://www.wdi.bus.umich.edu) 


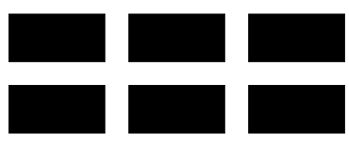

THE WILLIAM DAVIDSON INSTITUTE

AT THE UNIVERSITY OF MICHIGAN BUSINESSSCHOOL

\begin{tabular}{|c|c|c|}
\hline $\begin{array}{l}\text { No. } 314 \text { Is Life More Risky in the Open? } \\
\text { Household Risk-Coping and the Opening of } \\
\text { China's Labor Markets }\end{array}$ & John Giles & April 2000 \\
\hline $\begin{array}{l}\text { No. } 313 \text { Networks, Migration and Investment: } \\
\text { Insiders and Outsiders in Tirupur's } \\
\text { Production Cluster }\end{array}$ & Abhijit Banerjee and Kaivan Munshi & March 2000 \\
\hline $\begin{array}{l}\text { No. } 312 \text { Computational Analysis of the Impact } \\
\text { on India of the Uruguay Round and the } \\
\text { Forthcoming WTO Trade Negotiations }\end{array}$ & $\begin{array}{l}\text { Rajesh Chadha, Drusilla K. Brown, Alan } \\
\text { V. Deardorff and Robert M. Stern }\end{array}$ & March 2000 \\
\hline $\begin{array}{l}\text { No. } 311 \text { Subsidized Jobs for Unemployed } \\
\text { Workers in Slovakia }\end{array}$ & Jan. C. van Ours & May 2000 \\
\hline $\begin{array}{l}\text { No. } 310 \text { Determinants of Managerial Pay in } \\
\text { the Czech Republic }\end{array}$ & $\begin{array}{l}\text { Tor Eriksson, Jaromir Gottvald and Pavel } \\
\text { Mrazek }\end{array}$ & May 2000 \\
\hline $\begin{array}{l}\text { No. } 309 \text { The Great Human Capital } \\
\text { Reallocation: An Empirical Analysis of } \\
\text { Occupational Mobility in Transitional Russia }\end{array}$ & Klara Z. Sabirianova & May 2000 \\
\hline $\begin{array}{l}\text { No. } 308 \text { Economic Development, Legality, and } \\
\text { the Transplant Effect }\end{array}$ & $\begin{array}{l}\text { Daniel Berkowitz, Katharina Pistor, and } \\
\text { Jean-Francois Richard }\end{array}$ & February 2000 \\
\hline $\begin{array}{l}\text { No. } 307 \text { Community Participation, Teacher } \\
\text { Effort, and Educational Outcome: The Case of } \\
\text { El Salvador's EDUCO Program }\end{array}$ & Yasuyuki Sawada & November 1999 \\
\hline $\begin{array}{l}\text { No. } 306 \text { Gender Wage Gap and Segregation in } \\
\text { Late Transition }\end{array}$ & Stepan Jurajda & May 2000 \\
\hline $\begin{array}{l}\text { No. } 305 \text { The Gender Pay Gap in the } \\
\text { Transition from Communism: Some Empirical } \\
\text { Evidence }\end{array}$ & Andrew Newell and Barry Reilly & May 2000 \\
\hline $\begin{array}{l}\text { No. } 304 \text { Post-Unification Wage Growth in } \\
\text { East Germany }\end{array}$ & Jennifer Hunt & November 1998 \\
\hline $\begin{array}{l}\text { No. } 303 \text { How Does Privatization Affect } \\
\text { Workers? The Case of the Russian Mass } \\
\text { Privatization Program }\end{array}$ & Elizabeth Brainerd & May 2000 \\
\hline $\begin{array}{l}\text { No. } 302 \text { Liability for Past Environmental } \\
\text { Contamination and Privatization }\end{array}$ & Dietrich Earnhart & March 2000 \\
\hline No. 301 Varieties, Jobs and EU Enlargement & Tito Boeri and Joaquim Oliveira Martins & May 2000 \\
\hline No. 300 Employer Size Effects in Russia & Todd Idson & April 2000 \\
\hline $\begin{array}{l}\text { No. } 299 \text { Information Complements, } \\
\text { Substitutes, and Strategic Product Design }\end{array}$ & $\begin{array}{l}\text { Geoffrey G. Parker and Marshall W. Van } \\
\text { Alstyne }\end{array}$ & March 2000 \\
\hline $\begin{array}{l}\text { No. } 298 \text { Markets, Human Capital, and } \\
\text { Inequality: Evidence from Rural China }\end{array}$ & $\begin{array}{l}\text { Dwayne Benjamin, Loren Brandt, Paul } \\
\text { Glewwe, and Li Guo }\end{array}$ & May 2000 \\
\hline $\begin{array}{l}\text { No. } 297 \text { Corporate Governance in the Asian } \\
\text { Financial Crisis }\end{array}$ & $\begin{array}{l}\text { Simon Johnson, Peter Boone, Alasdair } \\
\text { Breach, and Eric Friedman }\end{array}$ & November 1999 \\
\hline $\begin{array}{l}\text { No. } 296 \text { Competition and Firm Performance: } \\
\text { Lessons from Russia }\end{array}$ & J. David Brown and John S. Earle & March 2000 \\
\hline $\begin{array}{l}\text { No. } 295 \text { Wage Determination in Russia: An } \\
\text { Econometric Investigation }\end{array}$ & Peter J. Luke and Mark E. Schaffer & March 2000 \\
\hline $\begin{array}{l}\text { No. 294: Can Banks Promote Enterprise } \\
\text { Restructuring?: Evidence From a Polish } \\
\text { Bank's Experience }\end{array}$ & John P. Bonin and Bozena Leven & March 2000 \\
\hline $\begin{array}{l}\text { No. 293: Why do Governments Sell Privatised } \\
\text { Companies Abroad? }\end{array}$ & $\begin{array}{l}\text { Bernardo Bortolotti, Marcella Fantini and } \\
\text { Carlo Scarpa }\end{array}$ & March 2000 \\
\hline No. 292: Going Public in Poland: Case-by- & Wolfgang Aussenegg & December 1999 \\
\hline
\end{tabular}

Publications denoted by an asterisk are not available on the Kresge Library webpage (http://www.wdi.bus.umich.edu) 


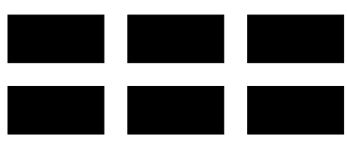

THE WILLIAM DAVIDSON INSTITUTE AT THE UNIVERSITY OF MICHIGAN BUSINESSSCHOOL

\begin{tabular}{|c|c|c|}
\hline $\begin{array}{l}\text { Case Privatizations, Mass Privatization and } \\
\text { Private Sector Initial Public Offerings }\end{array}$ & & \\
\hline $\begin{array}{l}\text { No. 291: Institutional Technology and the } \\
\text { Chains of Trust: Capital Markets and } \\
\text { Privatization in Russia and the Czech } \\
\text { Republic }\end{array}$ & Bruce Kogut and Andrew Spicer & March 1999 \\
\hline $\begin{array}{l}\text { No. 290: Banking Crises and Bank Rescues: } \\
\text { The Effect of Reputation }\end{array}$ & Jenny Corbett and Janet Mitchell & January 2000 \\
\hline $\begin{array}{l}\text { No. 289: Do Active Labor Market Policies } \\
\text { Help Unemployed Workers to Find and Keep } \\
\text { Regular Jobs? }\end{array}$ & Jan C. van Ours & February 2000 \\
\hline $\begin{array}{l}\text { No. 288: Consumption Patterns of the New } \\
\text { Elite in Zimbabwe }\end{array}$ & Russell Belk & February 2000 \\
\hline $\begin{array}{l}\text { No. 287: Barter in Transition Economies: } \\
\text { Competing Explanations Confront Ukranian } \\
\text { Data }\end{array}$ & $\begin{array}{l}\text { Dalia Marin, Daniel Kaufmann and } \\
\text { Bogdan Gorochowskij }\end{array}$ & January 2000 \\
\hline $\begin{array}{l}\text { No. 286: The Quest for Pension Reform: } \\
\text { Poland's Security through Diversity }\end{array}$ & Marek Góra and Michael Rutkowski & January 2000 \\
\hline $\begin{array}{l}\text { No. 285: Disorganization and Financial } \\
\text { Collapse }\end{array}$ & Dalia Marin and Monika Schnitzer & October 1999 \\
\hline $\begin{array}{l}\text { No. 284: Coordinating Changes in M-form } \\
\text { and U-form Organizations }\end{array}$ & $\begin{array}{l}\text { Yingyi Qian, Gérard Roland and } \\
\text { Chenggang } X u\end{array}$ & May 1999 \\
\hline $\begin{array}{l}\text { No. 283: Why Russian Workers Do Not Move: } \\
\text { Attachment of Workers Through In-Kind } \\
\text { Payments }\end{array}$ & Guido Friebel and Sergei Guriev & October 1999 \\
\hline $\begin{array}{l}\text { No. 282: Lessons From Fiascos in Russian } \\
\text { Corporate Governance }\end{array}$ & Merritt B. Fox and Michael A. Heller & October 1999 \\
\hline $\begin{array}{l}\text { No. 281: Income Distribution and Price } \\
\text { Controls: Targeting a Social Safety Net } \\
\text { During Economic Transition }\end{array}$ & Michael Alexeev and James Leitzel & March 1999 \\
\hline $\begin{array}{l}\text { No. 280: Starting Positions, Reform Speed, } \\
\text { and Economic Outcomes in Transitioning } \\
\text { Economies }\end{array}$ & William Hallagan and Zhang Jun & January 2000 \\
\hline No. 279: The Value of Prominent Directors & Yoshiro Miwa \& J. Mark Ramseyer & October 1999 \\
\hline No. 278: The System Paradigm & János Kornai & April 1998 \\
\hline $\begin{array}{l}\text { No. 277: The Developmental Consequences of } \\
\text { Foreign Direct Investment in the Transition } \\
\text { from Socialism to Capitalism: The } \\
\text { Performance of Foreign Owned Firms in } \\
\text { Hungary }\end{array}$ & Lawrence Peter King & September 1999 \\
\hline $\begin{array}{l}\text { No. 276: Stability and Disorder: An } \\
\text { Evolutionary Analysis of Russia's Virtual } \\
\text { Economy }\end{array}$ & Clifford Gaddy and Barry W. Ickes & November 1999 \\
\hline $\begin{array}{l}\text { No. 275: Limiting Government Predation } \\
\text { Through Anonymous Banking: A Theory with } \\
\text { Evidence from China. }\end{array}$ & $\begin{array}{l}\text { Chong-En Bai, David D. Li, Yingyi Qian } \\
\text { and Yijiang Wang }\end{array}$ & July 1999 \\
\hline *No. 274: Transition with Labour Supply & Tito Boeri & December 1999 \\
\hline $\begin{array}{l}\text { No. 273: Sectoral Restructuring and Labor } \\
\text { Mobility: A Comparative Look at the Czech } \\
\text { Republic }\end{array}$ & Vit Sorm and Katherine Terrell & November 1999 \\
\hline
\end{tabular}

Publications denoted by an asterisk are not available on the Kresge Library webpage (http://www.wdi.bus.umich.edu) 


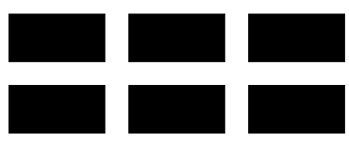

THE WILLIAM DAVIDSON INSTITUTE

AT THE UNIVERSITY OF MICHIGAN BUSINESSSCHOOL

\begin{tabular}{|c|c|c|}
\hline $\begin{array}{l}\text { *No. 272: Published in: Journal of } \\
\text { Comparative Economics "Returns to Human } \\
\text { Capital Under the Communist Wage Grid and } \\
\text { During the Transition to a Market Economy" } \\
\text { Vol. 27, pp. 33-60 1999. }\end{array}$ & $\begin{array}{l}\text { Daniel Munich, Jan Svejnar and Katherine } \\
\text { Terrell }\end{array}$ & October 1999 \\
\hline $\begin{array}{l}\text { No. 271: Barter in Russia: Liquidity Shortage } \\
\text { Versus Lack of Restructuring }\end{array}$ & Sophie Brana and Mathilde Maurel & June 1999 \\
\hline $\begin{array}{l}\text { No. 270: Tests for Efficient Financial } \\
\text { Intermediation with Application to China }\end{array}$ & Albert Park and Kaja Sehrt & March 1999 \\
\hline $\begin{array}{l}\text { No. 269a: Russian Privatization and } \\
\text { Corporate Governance: What Went Wrong? }\end{array}$ & $\begin{array}{l}\text { Bernard Black, Reinier Kraakman and } \\
\text { Anna Tarassova }\end{array}$ & May 2000 \\
\hline $\begin{array}{l}\text { No. 269: Russian Privatization and Corporate } \\
\text { Governance: What Went Wrong? }\end{array}$ & $\begin{array}{l}\text { Bernard Black, Reinier Kraakman and } \\
\text { Anna Tarassova }\end{array}$ & September 1999 \\
\hline $\begin{array}{l}\text { No. 268: Are Russians Really Ready for } \\
\text { Capitalism? }\end{array}$ & Susan Linz & September 1999 \\
\hline $\begin{array}{l}\text { No. 267: Do Stock Markets Promote } \\
\text { Economic Growth? }\end{array}$ & $\begin{array}{l}\text { Randall K. Filer, Jan Hanousek and Nauro } \\
\text { Campos }\end{array}$ & September 1999 \\
\hline $\begin{array}{l}\text { No. 266: Objectivity, Proximity and } \\
\text { Adaptability in Corporate Governance }\end{array}$ & Arnoud W.A Boot and Jonathan R. Macey & September 1999 \\
\hline $\begin{array}{l}\text { No. 265: When the Future is not What it Used } \\
\text { to Be: Lessons from the Western European } \\
\text { Experience to Forecasting Education and } \\
\text { Training in Transitional Economies }\end{array}$ & $\begin{array}{l}\text { Nauro F. Campos, Gerard Hughes, Stepan } \\
\text { Jurajda, and Daniel Munich }\end{array}$ & September 1999 \\
\hline $\begin{array}{l}\text { No. 264: The Institutional Foundation of } \\
\text { Foreign-Invested Enterprises (FIEs) in China }\end{array}$ & Yasheng Huang & September 1999 \\
\hline $\begin{array}{l}\text { No. 263: The Changing Corporate } \\
\text { Governance Paradigm: Implications for } \\
\text { Transition and Developing Countries }\end{array}$ & $\begin{array}{l}\text { Erik Berglof and Ernst-Ludwig von } \\
\text { Thadden }\end{array}$ & June 1999 \\
\hline No. 262: Law Enforcement and Transition & Gerard Roland and Thierry Verdier & May 1999 \\
\hline $\begin{array}{l}\text { No. 261: Soft Budget Constraints, Pecuniary } \\
\text { Externality, and the Dual Track System }\end{array}$ & Jiahua Che & June 2000 \\
\hline $\begin{array}{l}\text { No. 260: Missing Market in Labor Quality: } \\
\text { The Role of Quality Markets in Transiton }\end{array}$ & Gary H. Jefferson & July 1999 \\
\hline $\begin{array}{l}\text { No. 259: Do Corporate Global Environmental } \\
\text { Standards in Emerging Markets Create or } \\
\text { Destroy Market Value }\end{array}$ & $\begin{array}{l}\text { Glen Dowell, Stuart Hart and Bernard } \\
\text { Yeung }\end{array}$ & June 1999 \\
\hline $\begin{array}{l}\text { No. 258: Public Training and Outflows from } \\
\text { Unemployment }\end{array}$ & Patrick A. Puhani & June 1999 \\
\hline $\begin{array}{l}\text { No. 257: Ownership Versus Environment: } \\
\text { Why are Public Sector Firms Ineffecient? }\end{array}$ & Ann P. Bartel and Ann E. Harrison & June 1999 \\
\hline $\begin{array}{l}\text { No. 256: Taxation and Evasion in the } \\
\text { Presence of Exortion by Organized Crime }\end{array}$ & $\begin{array}{l}\text { Michael Alexeev, Eckhard Janeba and } \\
\text { Stefan Osborne }\end{array}$ & November 1999 \\
\hline $\begin{array}{l}\text { No. 255: Revisiting Hungary's Bankruptcy } \\
\text { Episode }\end{array}$ & John P. Bonin and Mark E. Schaffer & September 1999 \\
\hline $\begin{array}{l}\text { No. 254: FDI in Emerging Markets: A Home- } \\
\text { Country View }\end{array}$ & Marina v.N Whitman & June 1999 \\
\hline $\begin{array}{l}\text { No. 253: The Asian Financial Crisis: What } \\
\text { Happened, and What is to be Done }\end{array}$ & Jeffrey D. Sachs and Wing Thye Woo & January 1999 \\
\hline $\begin{array}{l}\text { No. 252: Organizational Law as Asset } \\
\text { Partitioning }\end{array}$ & Henry Hansmann and Reinier Kraakman & September 1999 \\
\hline No. 251: Consumer Behavior Research in & Jan-Benedict E. M. Steenkamp and Steven & September 1999 \\
\hline
\end{tabular}

Publications denoted by an asterisk are not available on the Kresge Library webpage (http://www.wdi.bus.umich.edu) 


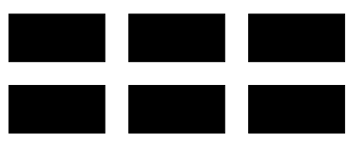

THE WILliam DAVIDSON InSTITUTE

AT THE UNIVERSITY OF MICHIGAN BUSINESSSCHOOL

\begin{tabular}{|c|c|c|}
\hline $\begin{array}{l}\text { Emerging Consumer Markets: the Case of the } \\
\text { Optimum Stimulation Level in South Africa }\end{array}$ & M. Burgess & \\
\hline $\begin{array}{l}\text { No. 250: Property Rights Formation and the } \\
\text { Organization of Exchange and Production in } \\
\text { Rural China }\end{array}$ & $\begin{array}{l}\text { Matthew A. Turner, Loren Brandt, and } \\
\text { Scott Rozelle }\end{array}$ & July 1998 \\
\hline $\begin{array}{l}\text { No. 249: Impacts of the Indonesian Economic } \\
\text { Crisis: Price Changes and the Poor }\end{array}$ & $\begin{array}{l}\text { James Levinsohn, Steven Berry, and Jed } \\
\text { Friedman }\end{array}$ & June 1999 \\
\hline $\begin{array}{l}\text { No. 248: Internal Barriers in the Transition of } \\
\text { Enterprises from Central Plan to Market }\end{array}$ & Charalambos Vlachoutsicos & July 1999 \\
\hline $\begin{array}{l}\text { No. 247: Spillovers from Multinationals in } \\
\text { Developing Countries: the Mechanisms at } \\
\text { Work }\end{array}$ & Richard E. Caves & June 1999 \\
\hline $\begin{array}{l}\text { No. 246: Dynamism and Inertia on the } \\
\text { Russian Labour Market: A Model of } \\
\text { Segmentation }\end{array}$ & $\begin{array}{l}\text { Irena Grosfeld, Claudia Senik-Leygonie, } \\
\text { Thierry Verdier, Stanislav Kolenikov and } \\
\text { Elena Paltseva }\end{array}$ & May 1999 \\
\hline $\begin{array}{l}\text { No. 245: Lessons from Bank Privatization in } \\
\text { Central Europe }\end{array}$ & John Bonin and Paul Wachtel & May 1999 \\
\hline $\begin{array}{l}\text { No. 244: Nominal-Real Tradeoffs and the } \\
\text { Effects of Monetary Policy: the Romanian } \\
\text { Experience }\end{array}$ & Christian Popa & December 1998 \\
\hline $\begin{array}{l}\text { No. 243: Privatization, Political Risk and } \\
\text { Stock Market Development in Emerging } \\
\text { Economies }\end{array}$ & Enrico C. Perotti and Pieter van Oijen & March 1999 \\
\hline $\begin{array}{l}\text { No. 242: Investment Financing in Russian } \\
\text { Financial-Industrial Groups }\end{array}$ & Enrico C. Perotti and Stanislav Gelfer & October 1998 \\
\hline $\begin{array}{l}\text { No. 241: Can governments maintain hard } \\
\text { budget constraints? Bank lending and } \\
\text { financial isolation in Romania }\end{array}$ & $\begin{array}{l}\text { Octavian Carare, Constantijn Claessens, } \\
\text { Enrico C. Perotti }\end{array}$ & January 1999 \\
\hline $\begin{array}{l}\text { No. 240: Democratic Institutions and } \\
\text { Economic Reform: the Polish Case }\end{array}$ & $\begin{array}{l}\text { John E. Jackson, Jacek Klich, and } \\
\text { Krystyna Poznanska }\end{array}$ & April 1998 \\
\hline $\begin{array}{l}\text { No. 239: A Longitudinal Study of IJV } \\
\text { Performance in Eastern Europe }\end{array}$ & Keith D. Brouthers and Gary Bamossy & June 1999 \\
\hline $\begin{array}{l}\text { No. 238: Published in: Journal of Business } \\
\text { Venturing, "Firm Creation and Economic } \\
\text { Transitions"” Vol. 14, Iss. 5, } 6 \text { Sep/Nov 1999, } \\
\text { pp. 427-450. }\end{array}$ & $\begin{array}{l}\text { John E. Jackson, Jacek Klich, Krystyna } \\
\text { Poznanska }\end{array}$ & July 1998 \\
\hline $\begin{array}{l}\text { No. 237: Analysis of Entrepreneurial Attitudes } \\
\text { in Poland }\end{array}$ & $\begin{array}{l}\text { John E. Jackson and Aleksander S. } \\
\text { Marcinkowski }\end{array}$ & March 1997 \\
\hline $\begin{array}{l}\text { No. 236: Investment and Finance in De Novo } \\
\text { Private Firms: Empirical Results from the } \\
\text { Czech Republic, Hungary, and Poland }\end{array}$ & $\begin{array}{l}\text { Andrzej Bratkowski, Irena Grosfeld, Jacek } \\
\text { Rostowski }\end{array}$ & April 1999 \\
\hline $\begin{array}{l}\text { No. 235: Does a Soft Macroeconomic } \\
\text { Environment Induce Restructuring on the } \\
\text { Microeconomic Level during the Transition } \\
\text { Period? Evidence from Investment Behavior } \\
\text { of Czech Enterprises }\end{array}$ & Lubomír Lizal & June 1999 \\
\hline $\begin{array}{l}\text { No. 234: Banking Reform in China: Gradually } \\
\text { Strengthening Pillar or Fragile Reed? }\end{array}$ & John Bonin & June 1999 \\
\hline $\begin{array}{l}\text { No. 233: Theories of Soft Budget Constraints } \\
\text { and the Analysis of Banking Crises }\end{array}$ & Janet Mitchell & March 1999 \\
\hline $\begin{array}{l}\text { No. 232: Unemployment Risk, Precautionary } \\
\text { Savings, and Moonlighting in Russia }\end{array}$ & $\begin{array}{l}\text { Alessandra Guariglia and Byung-Yeon } \\
\text { Kim }\end{array}$ & June 1999 \\
\hline
\end{tabular}

Publications denoted by an asterisk are not available on the Kresge Library webpage

(http://www.wdi.bus.umich.edu) 


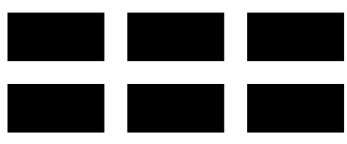

THE WILLIAM DAVIDSON INSTITUTE

AT THE UNIVERSITY OF MICHIGAN BUSINESSSCHOOL

\begin{tabular}{|c|c|c|}
\hline $\begin{array}{l}\text { No. 231: Investing in Turbulent Times: The } \\
\text { Investment Behavior of Polish Firms in the } \\
\text { Transition }\end{array}$ & $\begin{array}{l}\text { Josef C. Brada, Arthur E. King, and Chia- } \\
\text { Ying Ma }\end{array}$ & April 1999 \\
\hline $\begin{array}{l}\text { No. 230: The End of Moderate Inflation in } \\
\text { Three Transition Economies? }\end{array}$ & Josef C. Brada and Ali M. Kutan & April 1999 \\
\hline $\begin{array}{l}\text { No. 229: Back to the Future: The Growth } \\
\text { Prospects of Transition Economies } \\
\text { Reconsidered }\end{array}$ & Nauro F. Campos & April 1999 \\
\hline $\begin{array}{l}\text { No. 228: The Enterprise Isolation Program in } \\
\text { Russia }\end{array}$ & Simeon Djankov & April 1999 \\
\hline $\begin{array}{l}\text { No. 227: Published in: Journal of } \\
\text { Comparative Economics, "Ownership } \\
\text { Concentration and Corporate Performance in } \\
\text { the Czech Republic" 27(3), September 1999, } \\
\text { pp. 498-513. }\end{array}$ & Stijn Claessens and Simeon Djankov & April 1999 \\
\hline $\begin{array}{l}\text { No. 226: Unemployment Benefit Entitlement } \\
\text { and Training Effects in Poland during } \\
\text { Transition }\end{array}$ & Patrick A. Puhani & March 1999 \\
\hline $\begin{array}{l}\text { No. 225: Transition at Whirlpool-Tatramat: } \\
\text { Case Studies }\end{array}$ & Hans Brechbuhl and Sonia Ferencikova & March 1999 \\
\hline $\begin{array}{l}\text { No. 224: Measuring Progress in Transition } \\
\text { and Towards EU Accession: A Comparison of } \\
\text { Manufacturing Firms in Poland, Romania, } \\
\text { and Spain }\end{array}$ & $\begin{array}{l}\text { Wendy Carlin, Saul Estrin, and Mark } \\
\text { Schaffer }\end{array}$ & March 1999 \\
\hline $\begin{array}{l}\text { No. 223: Product Market Competition in } \\
\text { Transition Economies: Increasing Varieties } \\
\text { and Consumer Loyalty }\end{array}$ & Mitsutoshi M. Adachi & March 1999 \\
\hline $\begin{array}{l}\text { No. 222: Opaque Markets and Rapid Growth: } \\
\text { the Superiority of Bank-Centered Financial } \\
\text { Systems for Developing Nations }\end{array}$ & Rodney Wallace & July 1999 \\
\hline $\begin{array}{l}\text { No. 221: Technology Spillovers through } \\
\text { Foreign Direct Investment }\end{array}$ & Yuko Kinoshita & \begin{tabular}{|l|l} 
January 1999 \\
\end{tabular} \\
\hline $\begin{array}{l}\text { No. 220: Managerial, Expertise and Team } \\
\text { Centered Forms of Organizing: A Cross- } \\
\text { Cultural Exploration of Independence in } \\
\text { Engineering Work }\end{array}$ & Leslie Perlow & January 1999 \\
\hline $\begin{array}{l}\text { No. 219: Household Structure and Labor } \\
\text { Demand in Agriculture: Testing for } \\
\text { Separability in Rural China }\end{array}$ & Audra J. Bowlus and Terry Sicular & January 1999 \\
\hline $\begin{array}{l}\text { No. 218: Competing Strategies of FDI and } \\
\text { Technology Transfer to China: American and } \\
\text { Japanese Firms }\end{array}$ & W. Mark Fruin and Penelope Prime & January 1999 \\
\hline $\begin{array}{l}\text { No. } 217 \text { Published in: Journal of } \\
\text { Comparative Economics, "Returns to } \\
\text { Mobility in the Transition to a Market } \\
\text { Economy" Vol. 27, No. 1, March 1999, pp. 4- }\end{array}$ & Tito Boeri and Christopher J. Flinn & January 1999 \\
\hline $\begin{array}{l}\text { No. } 216 \text { Published in: Journal of } \\
\text { Comparative Economics, "Labor Market } \\
\text { Policies and Unemployment in the Czech } \\
\text { Republic." Vol. 27, No. 1, March 1999, pp. } \\
\text { 33-60. }\end{array}$ & Katherine Terrell and Vit Sorm & November 1998 \\
\hline No. 215 Published in: Journal of & Jochen Kluve, Hartmut Lehmann, and & December 1998 \\
\hline
\end{tabular}

Publications denoted by an asterisk are not available on the Kresge Library webpage (http://www.wdi.bus.umich.edu) 


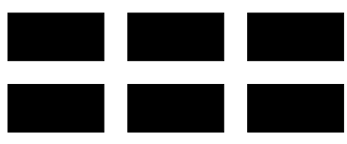

THE WILLIAM DAVIDSON INSTITUTE

AT THE UNIVERSITY OF MICHIGAN BUSINESSSCHOOL

\begin{tabular}{|c|c|c|}
\hline $\begin{array}{l}\text { Comparative Economics, "Active Labor } \\
\text { Market Policies in Poland: Human Capital } \\
\text { Enhancement, Stigmatization or Benefit } \\
\text { Churning?" Vol. 27, No. 1, March 1999, pp. } \\
61-\end{array}$ & Christoph M. Schmidt & \\
\hline $\begin{array}{l}\text { No. } 214 \text { Published in: Journal of } \\
\text { Comparative Economics, “Does the Slovenian } \\
\text { Public Work Program Increase Participants' } \\
\text { Chances to Find a Job?” Vol. 27, No.1, } \\
\text { March 1999, pp. 113- }\end{array}$ & Milan Vodopivec & December 1998 \\
\hline $\begin{array}{l}\text { No. } 213 \text { Published in: Journal of } \\
\text { Comparative Economics, "Effects of Active } \\
\text { Labor Market Programs on the Transition } \\
\text { Rate from Unemployment into Regular Jobs in } \\
\text { the Slovak Republic." Vol. 27, No. 1, March } \\
\text { 1999, pp. 90- }\end{array}$ & Martina Lubyova and Jan C. van Ours & December 1998 \\
\hline $\begin{array}{l}\text { No. 212: The Marketing System in Bulgarian } \\
\text { Livestock Production - The Present State and } \\
\text { Evolutionary Processes During the Period of } \\
\text { Economic Transition }\end{array}$ & Yordan Staykov, Team Leader & October 1998 \\
\hline $\begin{array}{l}\text { No. 211: Bankruptcy Experience in Hungary } \\
\text { and the Czech Republic }\end{array}$ & Janet Mitchell & October 1998 \\
\hline $\begin{array}{l}\text { No 210: Values, Optimum Stimulation Levels } \\
\text { and Brand Loyalty: New Scales in New } \\
\text { Populations }\end{array}$ & Steven M. Burgess and Mari Harris & September 1998 \\
\hline $\begin{array}{l}\text { No. 209: Inherited Wealth, Corporate Control } \\
\text { and Economic Growth }\end{array}$ & $\begin{array}{l}\text { Randall K. Morck, David A. Stangeland, } \\
\text { and Bernard Yeung }\end{array}$ & September 1998 \\
\hline $\begin{array}{l}\text { No. 208: A Cultural Analysis of Homosocial } \\
\text { Reproduction and Contesting Claims to } \\
\text { Competence in Transitional Firms }\end{array}$ & Michael D. Kennedy & July 1998 \\
\hline $\begin{array}{l}\text { No. 207: From Survival to Success: The } \\
\text { Journey of Corporate Transformation at } \\
\text { Haier. Forthcoming in Teaching the } \\
\text { Dinosaurs to Dance: Organizational Change } \\
\text { in Transition Economies ed. Daniel Denison. } \\
\text { No. 206: Why Do People Work If They Are } \\
\text { Not Paid? An Example from Eastern Europe. } \\
\text { Forthcoming in Teaching the Dinosaurs to } \\
\text { Dance: Organizational Change in Transition } \\
\text { Economies ed. Daniel Denison. }\end{array}$ & Arthur Yeung and Kenneth DeWoskin & $\begin{array}{l}\text { July } 1998 \\
\text { May } 1998\end{array}$ \\
\hline $\begin{array}{l}\text { No. 205: Firm Ownership and Work } \\
\text { Motivation in Bulgaria and Hungary: An } \\
\text { Empirical Study of the Transition in the Mid- } \\
\text { 1990s. Forthcoming in Teaching the } \\
\text { Dinosaurs to Dance: Organizational Change } \\
\text { in Transition Economies ed. Daniel Denison. }\end{array}$ & $\begin{array}{l}\text { Robert A. Roe, Irina L. Zinovieva, } \\
\text { Elizabeth Dienes, and Laurens A. ten Horn }\end{array}$ & May 1998 \\
\hline $\begin{array}{l}\text { No. 204: Human Resource Management in the } \\
\text { Restructuring of Chinese Joint Ventures. } \\
\text { Forthcoming in Teaching the Dinosaurs to } \\
\text { Dance: Organizational Change in Transition } \\
\text { Economies ed. Daniel Denison. }\end{array}$ & Nandani Lynton & April 1998 \\
\hline No. 203: Emergent Compensation Strategies & Marc Weinstein & March 1998 \\
\hline
\end{tabular}

Publications denoted by an asterisk are not available on the Kresge Library webpage (http://www.wdi.bus.umich.edu) 


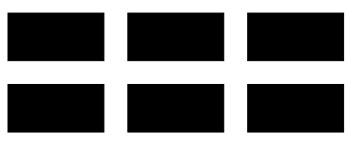

THE WILLIAM DAVIDSON INSTITUTE

AT THE UNIVERSITY OF MICHIGAN BUSINESSSCHOOL

\begin{tabular}{|c|c|c|}
\hline $\begin{array}{l}\text { in Post-Socialist Poland: Understanding the } \\
\text { Cognitive Underpinnings of Management } \\
\text { Practices in a Transition Economy. } \\
\text { Forthcoming in Teaching the Dinosaurs to } \\
\text { Dance: Organizational Change in Transition } \\
\text { Economies ed. Daniel Denison. }\end{array}$ & & \\
\hline $\begin{array}{l}\text { No. 202: Corporate Transformation and } \\
\text { Organizational Learning: The People's } \\
\text { Republic of China. Forthcoming in Teaching } \\
\text { the Dinosaurs to Dance: Organizational } \\
\text { Change in Transition Economies ed. Daniel } \\
\text { Denison. }\end{array}$ & Meinolf Dierkes and Zhang Xinhua & March 1998 \\
\hline $\begin{array}{l}\text { No. 201: Foreign Direct Investment as a } \\
\text { Factor of Change: The Case of Slovakia. } \\
\text { Forthcoming in Teaching the Dinosaurs to } \\
\text { Dance: Organizational Change in Transition } \\
\text { Economies ed. Daniel Denison. }\end{array}$ & Sonia Ferencikova & February 1998 \\
\hline $\begin{array}{l}\text { No. 200: Radical versus Incremental Change: } \\
\text { The Role of Capabilities, Competition, and } \\
\text { Leaders. Forthcoming in Teaching the } \\
\text { Dinosaurs to Dance: Organizational Change } \\
\text { in Transition Economies ed. Daniel Denison. }\end{array}$ & Karen L. Newman & February 1998 \\
\hline $\begin{array}{l}\text { No. 199: The Emergence of Market Practices } \\
\text { in China's Economic Transition: Price Setting } \\
\text { Practices in Shanghai's Industrial Firms. } \\
\text { Forthcoming in Teaching the Dinosaurs to } \\
\text { Dance: Organizational Change in Transition } \\
\text { Economies ed. Daniel Denison. }\end{array}$ & Douglas Guthrie & February 1998 \\
\hline $\begin{array}{l}\text { No. 198: The Application of Change } \\
\text { Management Methods at Business } \\
\text { Organizations Operating in Hungary: } \\
\text { Challenges in the Business and Cultural } \\
\text { Environment and First Practical Experiences. } \\
\text { Forthcoming in Teaching the Dinosaurs to } \\
\text { Dance: Organizational Change in Transition } \\
\text { Economies ed. Daniel Denison. }\end{array}$ & Dr. János Fehér & January 1998 \\
\hline $\begin{array}{l}\text { No. 197: Organizational Changes in Russian } \\
\text { Industrial Enterprises: Mutation of Decision- } \\
\text { Making Structures and Transformations of } \\
\text { Ownership. Forthcoming in Teaching the } \\
\text { Dinosaurs to Dance: Organizational Change } \\
\text { in Transition Economies ed. Daniel Denison. }\end{array}$ & Igor B. Gurkov & January 1998 \\
\hline $\begin{array}{l}\text { No. 196: Understanding and Managing } \\
\text { Challenges to the Romanian Companies } \\
\text { during Transition. Forthcoming in Teaching } \\
\text { the Dinosaurs to Dance: Organizational } \\
\text { Change in Transition Economies ed. Daniel } \\
\text { Denison. }\end{array}$ & Dan Candea and Rodica M. Candea & January 1998 \\
\hline $\begin{array}{l}\text { No. 195: Insider Lending and Economic } \\
\text { Transition: The Structure, Function, and } \\
\text { Performance Impact of Finance Companies in } \\
\text { Chinese Business Groups. Forthcoming in }\end{array}$ & Lisa A. Keister & December 1997 \\
\hline
\end{tabular}

Publications denoted by an asterisk are not available on the Kresge Library webpage (http://www.wdi.bus.umich.edu) 


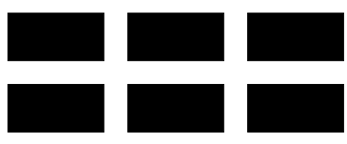

THE WILLIAM DAVIDSON INSTITUTE

AT THE UNIVERSITY OF MICHIGAN BUSINESSSCHOOL

\begin{tabular}{|c|c|c|}
\hline $\begin{array}{l}\text { Teaching the Dinosaurs to Dance: } \\
\text { Organizational Change in Transition } \\
\text { Economies ed. Daniel Denison. }\end{array}$ & & \\
\hline $\begin{array}{l}\text { No. 194: Japanese Investment in Transitional } \\
\text { Economies: Characteristics and Performance. } \\
\text { Forthcoming in Teaching the Dinosaurs to } \\
\text { Dance: Organizational Change in Transition } \\
\text { Economies ed. Daniel Denison. }\end{array}$ & Paul W. Beamish and Andrew Delios & November 1997 \\
\hline $\begin{array}{l}\text { No. 193: Building Successful Companies in } \\
\text { Transition Economies. Forthcoming in } \\
\text { Teaching the Dinosaurs to Dance: } \\
\text { Organizational Change in Transition } \\
\text { Economies ed. Daniel Denison. }\end{array}$ & Dr. Ivan Perlaki & January 1998 \\
\hline $\begin{array}{l}\text { No. 192: Russian Communitariansim: An } \\
\text { Invisible Fist in the Transformation Process of } \\
\text { Russia. Forthcoming in Teaching the } \\
\text { Dinosaurs to Dance: Organizational Change } \\
\text { in Transition Economies ed. Daniel Denison. }\end{array}$ & Charalambos Vlachoutsicos & July 1998 \\
\hline No. 191: Teaching the Dinosaurs to Dance & Michal Cakrt & September 1997 \\
\hline $\begin{array}{l}\text { No. 190: Strategic Restructuring: Making } \\
\text { Capitalism in Post-Communist Eastern } \\
\text { Europe. Forthcoming in Teaching the } \\
\text { Dinosaurs to Dance: Organizational Change } \\
\text { in Transition Economies ed. Daniel Denison. }\end{array}$ & Lawrence P. King & September 1997 \\
\hline $\begin{array}{l}\text { No. 189: Published in: Regional Science and } \\
\text { Urban Economics, “Russia's Internal } \\
\text { Border”, } 29 \text { (5), September } 1999 .\end{array}$ & Daniel Berkowitz and David N. DeJong & July 1998 \\
\hline $\begin{array}{l}\text { No. 187: Corporate Structure and } \\
\text { Performance in Hungary }\end{array}$ & László Halpern and Gábor Kórsöi & July 1998 \\
\hline $\begin{array}{l}\text { No. 186: Performance of Czech Companies by } \\
\text { Ownership Structure }\end{array}$ & Andrew Weiss and Georgiy Nikitin & June 1998 \\
\hline $\begin{array}{l}\text { No. 185: Firm Performance in Bulgaria and } \\
\text { Estonia: The effects of competitive pressure, } \\
\text { financial pressure and disorganisation }\end{array}$ & Jozef Konings & July 1998 \\
\hline $\begin{array}{l}\text { No. 184: Investment and Wages during the } \\
\text { Transition: Evidence from Slovene Firms }\end{array}$ & Janez Prasnikar and Jan Svejnar & July 1998 \\
\hline $\begin{array}{l}\text { No. 183: Investment Portfolio under Soft } \\
\text { Budget: Implications for Growth, Volatility } \\
\text { and Savings }\end{array}$ & Chongen Bai and Yijiang Wang & \\
\hline $\begin{array}{l}\text { No. 181: Delegation and Delay in Bank } \\
\text { Privatization }\end{array}$ & Loránd Ambrus-Lakatos and Ulrich Hege & July 1998 \\
\hline $\begin{array}{l}\text { No. 180: Financing Mechanisms and } R \& D \\
\text { Investment }\end{array}$ & Haizhou Huang and Chenggang Xu & July 1998 \\
\hline $\begin{array}{l}\text { No. 179: Organizational Culture and } \\
\text { Effectiveness: The Case of Foreign Firms in } \\
\text { Russia }\end{array}$ & Carl F. Fey and Daniel R. Denison & January 1999 \\
\hline $\begin{array}{l}\text { No. 178: Output and Unemployment } \\
\text { Dynamics in Transition }\end{array}$ & Vivek H. Dehejia and Douglas W. Dwyer & January 1998 \\
\hline $\begin{array}{l}\text { No. 177: Published in: Economics of } \\
\text { Transition,, "Bureaucracies in the Russian } \\
\text { Voucher Privatization" Vol. 8, No. 1, 2000, } \\
\text { pp. 37-57. }\end{array}$ & Guido Friebel & June 1998 \\
\hline
\end{tabular}

Publications denoted by an asterisk are not available on the Kresge Library webpage (http://www.wdi.bus.umich.edu) 


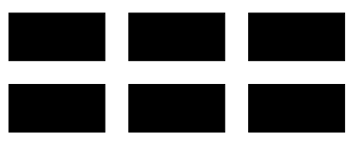

THE WILLIAM DAVIDSON INSTITUTE AT THE UNIVERSITY OF MICHIGAN BUSINESSSCHOOL

\begin{tabular}{|c|c|c|}
\hline $\begin{array}{l}\text { No. 176: Chronic Moderate Inflation in } \\
\text { Transition: The Tale of Hungary }\end{array}$ & János Vincze & June 1998 \\
\hline $\begin{array}{l}\text { No. 175: Privatisation and Market Structure } \\
\text { in a Transition Economy }\end{array}$ & John Bennett and James Maw & June 1998 \\
\hline $\begin{array}{l}\text { No. 174: Ownership and Managerial } \\
\text { Competition: Employee, Customer, or Outside } \\
\text { Ownership }\end{array}$ & Patrick Bolton and Chenggang Xu & June 1998 \\
\hline $\begin{array}{l}\text { No. 173: Intragovernment Procurement of } \\
\text { Local Public Good: A Theory of } \\
\text { Decentralization in Nondemocratic } \\
\text { Government }\end{array}$ & Chong-en Bai, Yu Pan and Yijiang Wang & June 1998 \\
\hline $\begin{array}{l}\text { No. 172: Political Instability and Growth in } \\
\text { Proprietary Economies }\end{array}$ & Jody Overland and Michael Spagat & August 1998 \\
\hline $\begin{array}{l}\text { No. 171: Published in Post-Communist } \\
\text { Economies, "Framework Issues in the } \\
\text { Privatization Strategies of the Czech Republic, } \\
\text { Hungary, and Poland" Vol. 11, no. 1 March } \\
\text { 1999. }\end{array}$ & Morris Bornstein & June 1998 \\
\hline $\begin{array}{l}\text { No. 170: Published in: European Journal of } \\
\text { Political Economy "Privatization, Ownership } \\
\text { Structure and Transparency: How to Measure } \\
\text { a Real Involvement of the State” 15(4), } \\
\text { November 1999, pp. 605-18. }\end{array}$ & Frantisek Turnovec & May 1998 \\
\hline $\begin{array}{l}\text { No. } 169 \text { Published in: American Economic } \\
\text { Review, “Unemployment and the Social Safety } \\
\text { Net during Transitions to a Market Economy: } \\
\text { Evidence from Czech and Slovak Men." Vol. } \\
\text { 88, No. 5, Dec. 1998, pp. 1117-1142. }\end{array}$ & $\begin{array}{l}\text { John C. Ham, Jan Svejnar, and Katherine } \\
\text { Terrell }\end{array}$ & December 1998 \\
\hline $\begin{array}{l}\text { No. 167: Voucher Privatization with } \\
\text { Investment Funds: An Institutional Analysis }\end{array}$ & David Ellerman & March 1998 \\
\hline $\begin{array}{l}\text { No. 166: Published in: Marketing Issues in } \\
\text { Transitional Economies, “Value Priorities } \\
\text { and Consumer Behavior in a Transitional } \\
\text { Economy: The Case of South Africa” ed. } \\
\text { Rajeev Batra. }\end{array}$ & $\begin{array}{l}\text { Steven M. Burgess and Jan-Benedict E.M. } \\
\text { Steenkamp }\end{array}$ & August 1998 \\
\hline $\begin{array}{l}\text { No. 164: Finance and Investment in } \\
\text { Transition: Czech Enterprises, 1993-1994 }\end{array}$ & Ronald Anderson and Chantal Kegels & September 1997 \\
\hline $\begin{array}{l}\text { No. 163: European Union Trade and } \\
\text { Investment Flows U-Shaping Industrial } \\
\text { Output in Central and Eastern Europe: } \\
\text { Theory and Evidence }\end{array}$ & Alexander Repkine and Patrick P. Walsh & April 1998 \\
\hline $\begin{array}{l}\text { No. 162: Skill Acquisition and Private Firm } \\
\text { Creation in Transition Economies }\end{array}$ & Zuzana Brixiova and Wenli Li & October 1999 \\
\hline No. 161: Corruption in Transition & Susanto Basu and David D. Li & May 1998 \\
\hline $\begin{array}{l}\text { No. 160a: Tenures that Shook the World: } \\
\text { Worker Turnover in Russia, Poland and } \\
\text { Britain }\end{array}$ & $\begin{array}{l}\text { Hartmut Lehmann and Jonathan } \\
\text { Wadsworth }\end{array}$ & November 1999 \\
\hline $\begin{array}{l}\text { No. 160: Tenures that Shook the World: } \\
\text { Worker Turnover in the Russian Federation } \\
\text { and Poland }\end{array}$ & $\begin{array}{l}\text { Hartmut Lehmann and Jonathan } \\
\text { Wadsworth }\end{array}$ & June 1998 \\
\hline $\begin{array}{l}\text { No. 159: Does Market Structure Matter? New } \\
\text { Evidence from Russia }\end{array}$ & Annette N. Brown and J. David Brown & June 1998 \\
\hline
\end{tabular}

Publications denoted by an asterisk are not available on the Kresge Library webpage (http://www.wdi.bus.umich.edu) 


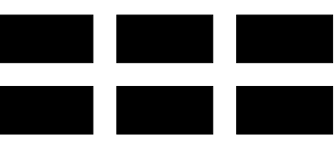

THE WILLIAM DAVIDSON INSTITUTE

AT THE UNIVERSITY OF MICHIGAN BUSINESSSCHOOL

\begin{tabular}{|c|c|c|}
\hline $\begin{array}{l}\text { No. 158: Structural Adjustment and Regional } \\
\text { Long Term Unemployment in Poland }\end{array}$ & Hartmut Lehmann and Patrick P. Walsh & June 1997 \\
\hline $\begin{array}{l}\text { No. 157: Baby Boom or Bust? Changing } \\
\text { Fertility in Post-Communist Czech Republic } \\
\text { and Slovakia }\end{array}$ & Robert S. Chase & April 1998 \\
\hline $\begin{array}{l}\text { No. } 156 \text { Published in: Leadership and } \\
\text { Organization Development Journal, } \\
\text { "Leading Radical Change in Transition } \\
\text { Economies." Vol. 19, No. 6, 1998, pp. 309- } \\
324 .\end{array}$ & Karen L. Newman & June 1998 \\
\hline $\begin{array}{l}\text { No. } 155 \text { Published in: Oxford Review of } \\
\text { Economic Policy, "From Theory into } \\
\text { Practice? Restructuring and Dynamism in } \\
\text { Transition Economies." Vol. 13, No. 2, } \\
\text { Summer 1997, pp. 77-105. }\end{array}$ & Wendy Carlin and Michael Landesmann & June 1997 \\
\hline $\begin{array}{l}\text { No. 154: The Model and the Reality: } \\
\text { Assessment of Vietnamese SOE Reform- } \\
\text { Implementation at the Firm Level }\end{array}$ & $\begin{array}{l}\text { Edmund Malesky, Vu Thanh Hung, Vu Thi } \\
\text { Dieu Anh, and Nancy K. Napier }\end{array}$ & July 1998 \\
\hline $\begin{array}{l}\text { No. } 153 \text { Published in: Journal of } \\
\text { Comparative Economics, "Causes of the Soft } \\
\text { Budget Constraint: Evidence on Three } \\
\text { Explanations." Vol. 26, No. 1, March 1998, } \\
\text { pp. 104-116. }\end{array}$ & David D. Li and Minsong Liang & March 1998 \\
\hline $\begin{array}{l}\text { No. } 152 \text { Published in: Comparative Economic } \\
\text { Studies, "Enterprise Restructuring in Russia's } \\
\text { Transition Economy: Formal and Informal } \\
\text { Mechanisms." Vol. 40, No. 2, Summer 1998, } \\
\text { pp. 5-52. }\end{array}$ & Susan J. Linz and Gary Krueger & April 1998 \\
\hline $\begin{array}{l}\text { No. 151: Labor Productivity in Transition: A } \\
\text { Regional Analysis of Russian Industry }\end{array}$ & Susan J. Linz & May 1998 \\
\hline $\begin{array}{l}\text { No. 150: Tax Avoidance and the Allocation of } \\
\text { Credit. Forthcoming in Financial Systems in } \\
\text { Transition: The Design of Financial Systems } \\
\text { in Central Europe eds. Anna Meyendorff and } \\
\text { Anjan Thakor. }\end{array}$ & Anna Meyendorff & June 1998 \\
\hline $\begin{array}{l}\text { No. 149: Commitment, Versatility and } \\
\text { Balance: Determinants of Work Time } \\
\text { Standards and Norms in a Multi-Country } \\
\text { Study of Software Engineers }\end{array}$ & Leslie Perlow and Ron Fortgang & April 1998 \\
\hline $\begin{array}{l}\text { No. 148: Changes in Poland's Transfer } \\
\text { Payments in the 1990s: the Fate of } \\
\text { Pensioners }\end{array}$ & Bozena Leven & June 1998 \\
\hline $\begin{array}{l}\text { No. 147: Environmental Protection and } \\
\text { Economic Development: The Case of the } \\
\text { Huaihe River Basin Cleanup Plan }\end{array}$ & $\begin{array}{l}\text { Robert Letovsky, Reze Ramazani, and } \\
\text { Debra Murphy }\end{array}$ & June 1998 \\
\hline $\begin{array}{l}\text { No. 146: Chief Executive Compensation } \\
\text { During Early Transition: Further Evidence } \\
\text { from Bulgaria }\end{array}$ & $\begin{array}{l}\text { Derek C. Jones, Takao Kato, and Jeffrey } \\
\text { Miller }\end{array}$ & June 1998 \\
\hline $\begin{array}{l}\text { No. } 145 \text { Published in: Economics of } \\
\text { Transition, “Women's Unemployment During } \\
\text { the Transition: Evidence from Czech and } \\
\text { Slovak Micro Data," Vol. 7, No. 1, May 1999, }\end{array}$ & $\begin{array}{l}\text { John Ham, Jan Svejnar, and Katherine } \\
\text { Terrell }\end{array}$ & May 1998 \\
\hline
\end{tabular}

Publications denoted by an asterisk are not available on the Kresge Library webpage (http://www.wdi.bus.umich.edu) 


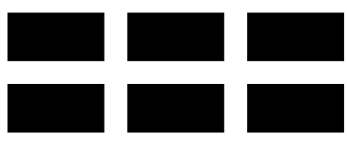

THE WILLIAM DAVIDSON INSTITUTE

AT THE UNIVERSITY OF MICHIGAN BUSINESSSCHOOL

\begin{tabular}{|c|c|c|}
\hline pp. 47-78. & & \\
\hline No. 144: Investment and Wages in Slovenia & Janez Prasnikar & May 1998 \\
\hline $\begin{array}{l}\text { No. } 143 \text { Published in: Review of Financial } \\
\text { Studies, “Optimal Bankruptcy Laws Across } \\
\text { Different Economic Systems,” 12(2), Summer } \\
\text { 1999, pgs. 347-77. }\end{array}$ & Elazar Berkovitch and Ronen Israel & March 1998 \\
\hline $\begin{array}{l}\text { No. 142: Industrial Policy and Poverty in } \\
\text { Transition Economies: Two Steps Forward or } \\
\text { One Step Back? }\end{array}$ & Susan J. Linz & March 1998 \\
\hline $\begin{array}{l}\text { No. 141: Collective Ownership and } \\
\text { Privatization of China's Village Enterprises }\end{array}$ & Suwen Pan and Albert Park & April 1998 \\
\hline $\begin{array}{l}\text { No. 140: A Comparative Look at Labor } \\
\text { Mobility in the Czech Republic: Where have } \\
\text { all the Workers Gone? }\end{array}$ & Vit Sorm and Katherine Terrell & April 1999 \\
\hline $\begin{array}{l}\text { No. 139: The Failure of the Government-Led } \\
\text { Program of Corporate Reorganization in } \\
\text { Romania }\end{array}$ & Simeon Djankov and Kosali Ilayperuma & September 1997 \\
\hline $\begin{array}{l}\text { No. 138: Ownership and Employment in } \\
\text { Russian Industry: 1992-1995 }\end{array}$ & Susan J. Linz & March 1998 \\
\hline $\begin{array}{l}\text { No. } 137 \text { Published in: Journal of Political } \\
\text { Economy, "Reform Without Losers: An } \\
\text { Interpretation of China's Dual-Track } \\
\text { Approach to Transition," Feb. 2000; Vol. 108, } \\
\text { Iss.1; pg. } 120\end{array}$ & $\begin{array}{l}\text { Lawrence J. Lau, Yingyi Qian, and Gerard } \\
\text { Roland }\end{array}$ & November 1997 \\
\hline $\begin{array}{l}\text { No. } 136 \text { Published in: European Economic } \\
\text { Review, "The Political Economy of Mass } \\
\text { Privatization and the Risk of Expropriation," } \\
44(2), \text { February 2000, pgs. 393-421 }\end{array}$ & Klaus M. Schmidt & March 1998 \\
\hline $\begin{array}{l}\text { No. 135: Radical Organizational Change: The } \\
\text { Role of Starting Conditions, Competition, and } \\
\text { Leaders }\end{array}$ & Karen L. Newman & January 1998 \\
\hline $\begin{array}{l}\text { No. 134: To Restructure or Not to } \\
\text { Restructure: Informal Activities and } \\
\text { Enterprise Behavior in Transition }\end{array}$ & Clifford Gaddy and Barry W. Ickes & May 1998 \\
\hline $\begin{array}{l}\text { No. 133: Management 101: Behavior of Firms } \\
\text { in Transition Economies }\end{array}$ & Josef C. Brada & March 1998 \\
\hline $\begin{array}{l}\text { No. } 132 \text { Published in: Quarterly Journal of } \\
\text { Economics, "Interfirm Relationships and } \\
\text { Informal Credit in Vietnam," 114(4), Nov. } \\
\text { 1999, pgs. 1285-1320 }\end{array}$ & John McMillan and Christopher Woodruff & February 1998 \\
\hline $\begin{array}{l}\text { No. } 131 \text { Published in: Comparative Economic } \\
\text { Studies, “Will Restructuring Hungarian } \\
\text { Companies Innovate? An Investigation Based } \\
\text { on Joseph Berliner's Analysis of Innovation in } \\
\text { Soviet Industry.” Vol. 40, No. 2, Summer } \\
\text { 1998, pp. 53-74. }\end{array}$ & John B. Bonin and Istvan Abel & March 1998 \\
\hline $\begin{array}{l}\text { No. 130: Published in The American } \\
\text { Economic Review, “Changing Incentives of } \\
\text { the Chinese Bureaucracy.” May, } 1998 .\end{array}$ & David D. Li & January 1998 \\
\hline $\begin{array}{l}\text { No. 129: Restructuring Investment in } \\
\text { Transition: A Model of the Enterprise } \\
\text { Decision }\end{array}$ & Richard E. Ericson & January 1998 \\
\hline
\end{tabular}

Publications denoted by an asterisk are not available on the Kresge Library webpage (http://www.wdi.bus.umich.edu) 


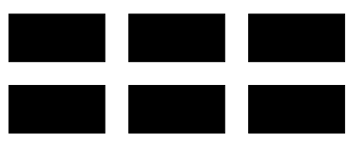

THE WILLIAM DAVIDSON INSTITUTE

AT THE UNIVERSITY OF MICHIGAN BUSINESSSCHOOL

\begin{tabular}{|c|c|c|}
\hline $\begin{array}{l}\text { No. } 128 \text { Published in: Comparative Economic } \\
\text { Studies, "Job Rights in Russian Firms: } \\
\text { Endangered or Extinct Institutions?" Vol. 40, } \\
\text { No. 4, Winter 1998, pp. 1-32. }\end{array}$ & Susan J. Linz & January 1998 \\
\hline $\begin{array}{l}\text { No. 127: Accounting for Growth in Post- } \\
\text { Soviet Russia }\end{array}$ & Daniel Berkowitz and David N. DeJong & January 1998 \\
\hline $\begin{array}{l}\text { No. } 126 \text { Published in: Economics of } \\
\text { Transition, "From Federalism, Chinese Style, } \\
\text { to Privatization Chinese Style," 7(1), 1999, } \\
\text { pgs. 103-31 }\end{array}$ & $\begin{array}{l}\text { Yuanzheng Cao, Yingyi Qian, and Barry R. } \\
\text { Weingast }\end{array}$ & December 1997 \\
\hline $\begin{array}{l}\text { No. 125: Market Discipline in Conglomerate } \\
\text { Banks: Is an Internal Allocation of Cost of } \\
\text { Capital Necessary as Incentive Device? } \\
\text { Forthcoming in Financial Systems in } \\
\text { Transition: The Design of Financial Systems } \\
\text { in Central Europe eds. Anna Meyendorff and } \\
\text { Anjan Thakor. }\end{array}$ & Arnoud W. A. Boot and Anjolein Schmeits & November 1997 \\
\hline $\begin{array}{l}\text { No. 124: Financial Discipline in the } \\
\text { Enterprise Sector in Transition Countries: } \\
\text { How Does China Compare? }\end{array}$ & Shumei Gao and Mark E. Schaffer & February 1998 \\
\hline $\begin{array}{l}\text { No. 123: Considerations of an Emerging } \\
\text { Marketplace: Managers' Perceptions in the } \\
\text { Southern African Economic Community }\end{array}$ & Brent Chrite and David Hudson & February 1998 \\
\hline $\begin{array}{l}\text { No. 122: A Model of the Informal Economy in } \\
\text { Transition Economies }\end{array}$ & $\begin{array}{l}\text { Simon Commander and Andrei } \\
\text { Tolstopiatenko }\end{array}$ & November 1997 \\
\hline $\begin{array}{l}\text { No. 121: Local Labour Market Dynamics in } \\
\text { the Czech and Slovak Republics }\end{array}$ & Peter Huber and Andreas Worgotter & November 1997 \\
\hline $\begin{array}{l}\text { No. 121: Local Labour Market Dynamics in } \\
\text { the Czech and Slovak Republics }\end{array}$ & Peter Huber and Andreas Worgotter & November 1997 \\
\hline $\begin{array}{l}\text { No. 119: Institutional Upheaval and Company } \\
\text { Transformation in Emerging Market } \\
\text { Economies }\end{array}$ & Karen L. Newman & March 1998 \\
\hline $\begin{array}{l}\text { No. 118: Industrial Decline and Labor } \\
\text { Reallocation in Romania }\end{array}$ & John S. Earle & October 1997 \\
\hline $\begin{array}{l}\text { No. 117: Notes for an Essay on the Soft } \\
\text { Budget Constraint }\end{array}$ & Lorand Ambrus-Lakatos & January 1997 \\
\hline $\begin{array}{l}\text { No. 116: Labor Demand During Transition in } \\
\text { Hungary }\end{array}$ & Gabor Korosi & October 1997 \\
\hline $\begin{array}{l}\text { No. 115: Enterprise Performance and } \\
\text { Managers' Profiles }\end{array}$ & Simeon Djankov and Stijn Claessens & December 1997 \\
\hline $\begin{array}{l}\text { No. } 114 \text { bmployment and Wages in } \\
\text { Enterprises under Communism and in } \\
\text { Transition: Evidence From Central Europe } \\
\text { and Russia }\end{array}$ & Swati Basu, Saul Estrin, and Jan Svejnar & April 2000 \\
\hline $\begin{array}{l}\text { No. 114: Employment and Wage Behavior of } \\
\text { Enterprises in Transitional Economies }\end{array}$ & Swati Basu, Saul Estrin, and Jan Svejnar & October 1997 \\
\hline $\begin{array}{l}\text { No. 113: Preliminary Evidence on Active } \\
\text { Labor Programs' Impact in Hungary and } \\
\text { Poland }\end{array}$ & Christopher J. O’Leary & October 1997 \\
\hline $\begin{array}{l}\text { No. 111: Unemployment Benefits and } \\
\text { Incentives in Hungary: New Evidence }\end{array}$ & Joachim Wolff & October 1997 \\
\hline No. 110: Published in: Empirical Economics, & Marek Gora and Christoph M. Schmidt & April 1997 \\
\hline
\end{tabular}

Publications denoted by an asterisk are not available on the Kresge Library webpage

(http://www.wdi.bus.umich.edu) 


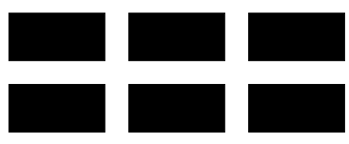

THE WILLIAM DAVIDSON INSTITUTE

AT THE UNIVERSITY OF MICHIGAN BUSINESSSCHOOL

\begin{tabular}{|c|c|c|}
\hline $\begin{array}{l}\text { "Long-Term Unemployment, Unemployment } \\
\text { Benefits and Social Assistance: The Polish } \\
\text { Experience" Empirical-Economics; 23(1-2), } \\
\text { 1998, pages 55-85. }\end{array}$ & & \\
\hline $\begin{array}{l}\text { No. } 109 \text { Published in: Industrial and Labor } \\
\text { Relations Review, "Markets for Communist } \\
\text { Human Capital: Returns to Education and } \\
\text { Experience in Post-Communist Czech } \\
\text { Republic and Slovakia." Vol. 51, No. 3, April } \\
\text { 1998, pp. 401-423. }\end{array}$ & Robert S. Chase & October 1997 \\
\hline $\begin{array}{l}\text { No. 107: The Worker-Firm Matching in the } \\
\text { Transition: (Why) Are the Czechs More } \\
\text { Successful Than Others? }\end{array}$ & $\begin{array}{l}\text { Daniel Münich, Jan Svejnar, and } \\
\text { Katherine Terrell }\end{array}$ & October 1997 \\
\hline $\begin{array}{l}\text { No. } 106 \text { Published in: Journal of } \\
\text { Comparative Economics, "Job Creation, Job } \\
\text { Destruction and Growth of Newly Established, } \\
\text { Privatized and State-Owned Enterprises in } \\
\text { Transition Economies: Survey Evidence from } \\
\text { Bulgaria, Hungary, and Romania," Vol. 26, } \\
\text { No.3, September 1998, pp. 429-445. }\end{array}$ & Valentijn Bilsen and Jozef Konings & September 1998 \\
\hline $\begin{array}{l}\text { No. 105: Getting Behind the East-West } \\
\text { [German] Wage Differential: Theory and } \\
\text { Evidence }\end{array}$ & Michael Burda and Christoph Schmidt & May 1997 \\
\hline $\begin{array}{l}\text { No. 104: The Birth of the "Wage Curve" in } \\
\text { Hungary, 1989-95 }\end{array}$ & Gabor Kertesi and Janos Kollo & October 1997 \\
\hline $\begin{array}{l}\text { No. 103: Published in: Journal of } \\
\text { Comparative Economics, "Grime and } \\
\text { Punishment: Job Insecurity and Wage Arrears } \\
\text { in the Russian Federation" 27, 595-617 } \\
\text { (1999). }\end{array}$ & $\begin{array}{l}\text { Hartmut Lehmann, Jonathan Wadsworth, } \\
\text { and Alessandro Acquisti }\end{array}$ & October 1997 \\
\hline No. 102: Social Networks in Transition & $\begin{array}{l}\text { Lorena Barberia, Simon Johnson, and } \\
\text { Daniel Kaufmann }\end{array}$ & October 1997 \\
\hline $\begin{array}{l}\text { No. 101: Depreciation and Russian Corporate } \\
\text { Finance: A Pragmatic Approach to Surviving } \\
\text { the Transition }\end{array}$ & Susan J. Linz & November 1997 \\
\hline No. 100: Romanian Financial System Reform & Anna Meyendorff and Anjan V. Thakor & November 1997 \\
\hline $\begin{array}{l}\text { No. 99: Proceedings of the Conference on } \\
\text { Strategic Alliances in Transitional Economies, } \\
\text { held May 20, } 1997 \text { at the Davidson Institute }\end{array}$ & Edited by Cynthia Koch & May 1997 \\
\hline $\begin{array}{l}\text { No. 98: Institutions, Strain and the } \\
\text { Underground Economy }\end{array}$ & Daniel Daianu and Lucian Albu & November 1997 \\
\hline $\begin{array}{l}\text { No. 97: Structure and Strain in Explaining } \\
\text { Inter-Enterprise Arrears } \\
\text { No. 96: Resource Misallocation and Strain: } \\
\text { Explaining Shocks in Post-Command } \\
\text { Economies }\end{array}$ & $\begin{array}{l}\text { Daniel Daianu } \\
\text { Daniel Daianu }\end{array}$ & $\begin{array}{l}\text { November } 1997 \\
\text { November } 1997\end{array}$ \\
\hline $\begin{array}{l}\text { No. 95: Published in: Finance-a-Uver, } \\
\text { "Czech Money Market: Emerging Links } \\
\text { Among Interest Rates." 48(2) } 1998 \text { pp. 99- } \\
\text { 109. }\end{array}$ & Jan Hanousek and Evzen Kocenda & November 1997 \\
\hline $\begin{array}{l}\text { No. 94: Pre-Reform Industry and the } \\
\text { State Monopsony in China }\end{array}$ & Xiao-Yuan Dong and Louis Putterman & October 1997 \\
\hline
\end{tabular}

Publications denoted by an asterisk are not available on the Kresge Library webpage (http://www.wdi.bus.umich.edu) 


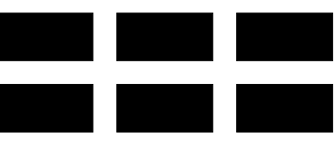

THE WILLIAM DAVIDSON INSTITUTE AT THE UNIVERSITY OF MICHIGAN BUSINESSSCHOOL

\begin{tabular}{|c|c|c|}
\hline $\begin{array}{l}\text { No. 93: China's State-Owned Enterprises } \\
\text { In the First Reform Decade: } \\
\text { An Analysis of a Declining Monopsony }\end{array}$ & Xiao-Yuan Dong and Louis Putterman & October 1997 \\
\hline $\begin{array}{l}\text { No. 92: Expatriate Management in the Czech } \\
\text { Republic }\end{array}$ & Richard B. Peterson & September 1997 \\
\hline $\begin{array}{l}\text { No. 91: China and the Idea of Economic } \\
\text { Reform }\end{array}$ & Thomas G. Rawski & April 1997 \\
\hline $\begin{array}{l}\text { No. } 90 \text { Published in: China Economic } \\
\text { Review, “China's State Enterprise Reform: An } \\
\text { Overseas Perspective." Vol. 8, Spring 1997, } \\
\text { pp. 89-98. }\end{array}$ & Thomas G. Rawski & July 1997 \\
\hline $\begin{array}{l}\text { No. 89: The Economic Determinants of } \\
\text { Internal Migration Flows in Russia During } \\
\text { Transition }\end{array}$ & Annette N. Brown & July 1997 \\
\hline $\begin{array}{l}\text { No. 88: Gender Wage Gaps in China's Labor } \\
\text { Market: Size, Structure, Trends }\end{array}$ & $\begin{array}{l}\text { Margaret Maurer-Fazio, Thomas G. } \\
\text { Rawski, and Wei Zhang }\end{array}$ & July 1997 \\
\hline $\begin{array}{l}\text { No. 87: Privatisation in Central and Eastern } \\
\text { Europe }\end{array}$ & Saul Estrin & June 1997 \\
\hline $\begin{array}{l}\text { No. 86: Published in : Economics of } \\
\text { Transition, "The Effect of Privatization on } \\
\text { Wealth Distribution in Russia." v. 7, no. 2, } \\
\text { 1999, pp. 449-65 }\end{array}$ & Michael Alexeev & February 1998 \\
\hline $\begin{array}{l}\text { No. 85: Was Privatization in Eastern Germany } \\
\text { a Special Case? Some Lessons from the } \\
\text { Treuhand }\end{array}$ & Uwe Siegmund & September 1997 \\
\hline No. 84: Start-ups and Transition & Daniel M. Berkowitz and David J. Cooper & September 1997 \\
\hline $\begin{array}{l}\text { No. 83: Which Enterprises (Believe They) } \\
\text { Have Soft Budgets after Mass Privatization? } \\
\text { Evidence from Mongolia }\end{array}$ & $\begin{array}{l}\text { James Anderson, Georges Korsun, and } \\
\text { Peter Murrell }\end{array}$ & October 1997 \\
\hline $\begin{array}{l}\text { No. 82: Published in: European Economic } \\
\text { Review, "Unemployment Dynamics and the } \\
\text { Restructuring of the Slovak Unemployment } \\
\text { Benefit System." April, } 1997 .\end{array}$ & Martina Lubyova and Jan C. van Ours & June 1997 \\
\hline $\begin{array}{l}\text { No. 81: Determinants of Unemployment } \\
\text { Duration in Russia }\end{array}$ & Mark C. Foley & August 1997 \\
\hline $\begin{array}{l}\text { No. 80: The Many Faces of Information } \\
\text { Disclosure }\end{array}$ & Arnoud W.A. Boot and Anjan V. Thakor & October 1997 \\
\hline $\begin{array}{l}\text { No. 79: Published in: Journal of Finance, } \\
\text { "Foreign Speculators and Emerging Equity } \\
\text { Markets."v.22, iss. 2, 2000, pp. 565-613 }\end{array}$ & Geert Bekaert and Campbell R. Harvey & August 1997 \\
\hline $\begin{array}{l}\text { No. 78: The Relationship Between Economic } \\
\text { Factors and Equity Markets in Central Europe }\end{array}$ & Jan Hanousek and Randall K. Filer & June 1997 \\
\hline $\begin{array}{l}\text { No. } 77 \text { Published in: Economics of } \\
\text { Transition, “A Gini Decomposition Analysis } \\
\text { of Inequality in the Czech and Slovak } \\
\text { Republics During the Transition," Vol. 6, } \\
\text { No.1, May 1998, pp. 23-46. }\end{array}$ & Thesia I. Garner and Katherine Terrell & May 1998 \\
\hline $\begin{array}{l}\text { No. 76: China's Emerging Market for } \\
\text { Property Rights: Theoretical and Empirical } \\
\text { Perspectives }\end{array}$ & Gary H. Jefferson and Thomas G. Rawski & June 1997 \\
\hline $\begin{array}{l}\text { No. } 75 b: \text { Test of Permanent Income } \\
\text { Hypothesis on Czech Voucher Privatization }\end{array}$ & Jan Hanousek and Zdenek Tima & October 1997 \\
\hline
\end{tabular}

Publications denoted by an asterisk are not available on the Kresge Library webpage (http://www.wdi.bus.umich.edu) 


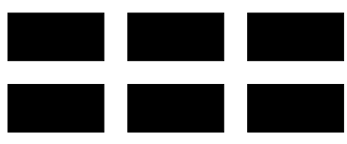

THE WILLIAM DAVIDSON INSTITUTE

AT THE UNIVERSITY OF MICHIGAN BUSINESSSCHOOL

\begin{tabular}{|c|c|c|}
\hline $\begin{array}{l}\text { No. 74: Determinants of Performance of } \\
\text { Manufacturing Firms in Seven European } \\
\text { Transition Economies }\end{array}$ & $\begin{array}{l}\text { Stijn Claessens, Simeon Djankov, and } \\
\text { Gerhard Pohl }\end{array}$ & February 1997 \\
\hline $\begin{array}{l}\text { No. } 73 \text { Published in: Economics of } \\
\text { Transition, "The Restructuring of Large } \\
\text { Firms in Slovak Republic." Vol. 6, No. 1, May } \\
\text { 1998, pp. 67-85 }\end{array}$ & Simeon Djankov and Gerhard Pohl & May 1998 \\
\hline $\begin{array}{l}\text { No. 72: Law, Relationships, and Private } \\
\text { Enforcement: Transactional Strategies of } \\
\text { Russian Enterprises }\end{array}$ & $\begin{array}{l}\text { Kathryn Hendley, Peter Murrell, and } \\
\text { Randi Ryterman }\end{array}$ & November 1998 \\
\hline $\begin{array}{l}\text { No. 71: Giving Credit Where Credit Is Due: } \\
\text { The Changing Role of Rural Financial } \\
\text { Institutions in China }\end{array}$ & Albert Park, Loren Brandt, and John Giles & March 1997 \\
\hline $\begin{array}{l}\text { No. } 70: \text { Privatization Versus Competition: } \\
\text { Changing Enterprise Behavior in Russia }\end{array}$ & John S. Earle and Saul Estrin & Spring 1997 \\
\hline $\begin{array}{l}\text { No. 69: Russian Managers under Storm: } \\
\text { Explicit Reality and Implicit Leadership } \\
\text { Theories (A Pilot Exploration) }\end{array}$ & Igor Gurkov & October 1998 \\
\hline $\begin{array}{l}\text { No. 68: The Political Economy of Central- } \\
\text { Local Relations in China: Inflation and } \\
\text { Investment Controls During the Reform Era }\end{array}$ & Yasheng Huang & Spring 1997 \\
\hline $\begin{array}{l}\text { No. 67: Between Two Coordination Failures: } \\
\text { Automotive Industrial Policy in China with a } \\
\text { Comparison to Korea }\end{array}$ & Yasheng Huang & Spring 1997 \\
\hline $\begin{array}{l}\text { No. } 66 \text { Published in: Post-Soviet Geography } \\
\text { and Economics, "Red Executives in Russia's } \\
\text { Transition Economy." Vol. 27, No. 10, } \\
\text { November 1996, pp. 633-651. }\end{array}$ & Susan J. Linz & January 1997 \\
\hline $\begin{array}{l}\text { No. } 65 \text { Published in: Industrial and } \\
\text { Corporate Change, "On the Sequencing of } \\
\text { Privatization in Transition Economies." Vol. } \\
7, \text { No. } 1,1998 .\end{array}$ & Gautam Ahuja and Sumit K. Majumdar & April 1997 \\
\hline $\begin{array}{l}\text { No. 64: Published in: Journal of Law and } \\
\text { Economics, "Foreign Ownership and } \\
\text { Profitability: Property Rights, Control and the } \\
\text { Performance of Firms in Indian Industry" } \\
\text { 42(1), April 1999, pp. 209-38. }\end{array}$ & $\begin{array}{l}\text { Pradeep K. Chhibber and Sumit K. } \\
\text { Majumdar }\end{array}$ & April 1997 \\
\hline $\begin{array}{l}\text { No. 63: How Taxing Is Corruption on } \\
\text { International Investors? }\end{array}$ & Shang-Jin Wei & February 1997 \\
\hline $\begin{array}{l}\text { No. 62: What Can We Learn from the } \\
\text { Experience of Transitional Economies with } \\
\text { Labour Market Policies? } \\
\text { No. 61: Published in: Accounting } \\
\text { Organizations and Society, "Economic } \\
\text { Transition, Strategy and the Evolution of } \\
\text { Management Accounting Practices: The Case } \\
\text { of India" 24(5,6), Jul/Aug 1999, pp. 379-412. }\end{array}$ & $\begin{array}{l}\text { Tito Boeri } \\
\text { Shannon W. Anderson and William N. } \\
\text { Lanen }\end{array}$ & 1997 \\
\hline $\begin{array}{l}\text { No. 60a: Enterprise Investment During the } \\
\text { Transition: Evidence from Czech Panel Data }\end{array}$ & Lubomír Lizal and Jan Svejnar & December 1997 \\
\hline $\begin{array}{l}\text { No. 59: Published in: Journal of Law, } \\
\text { Economics, and Organization, "Institutional } \\
\text { Environment, Community Government, and }\end{array}$ & Jiahua Che and Yingyi Qian & April 1997 \\
\hline
\end{tabular}

Publications denoted by an asterisk are not available on the Kresge Library webpage (http://www.wdi.bus.umich.edu) 


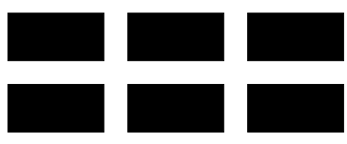

THE WILLIAM DAVIDSON INSTITUTE

AT THE UNIVERSITY OF MICHIGAN BUSINESSSCHOOL

\begin{tabular}{|c|c|c|}
\hline $\begin{array}{l}\text { Corporate Governance: Understanding } \\
\text { China's Township-Village Enterprises." } \\
\text { 14(1), April 1998, pages 1-23 }\end{array}$ & & \\
\hline $\begin{array}{l}\text { No. 58: From the Grabbing Hand to the } \\
\text { Helping Hand }\end{array}$ & Jiahua Che & June 2000 \\
\hline $\begin{array}{l}\text { No. 57: Published in: Brookings Papers on } \\
\text { Economic Activity, “The Unofficial Economy } \\
\text { in Transition." 1: 1998. }\end{array}$ & $\begin{array}{l}\text { Simon Johnson, Daniel Kaufmann, and } \\
\text { Andrei Schleifer }\end{array}$ & June 1997 \\
\hline $\begin{array}{l}\text { No. 56: Taxes and Government Incentives: } \\
\text { Eastern Europe vs. China }\end{array}$ & Roger H. Gordon and David D. Li & April 1997 \\
\hline No. 55: Corruption and Reform & Susanto Basu and David Li & June 1996 \\
\hline $\begin{array}{l}\text { No. 54: Decentralization and the } \\
\text { Macroeconomic Consequences of } \\
\text { Commitment to State-Owned Firms }\end{array}$ & Loren Brandt and Xiaodong Zhu & June 1997 \\
\hline $\begin{array}{l}\text { No. 53: Published in: The International } \\
\text { Journal of Industrial Organization, } \\
\text { "Competitive Shocks and Industrial Structure: } \\
\text { The Case of Polish Manufacturing." August, } \\
\text { 1999. . }\end{array}$ & Pankaj Ghemawat and Robert E. Kennedy & May 1997 \\
\hline $\begin{array}{l}\text { No. 52: Published in: The Quarterly Journal } \\
\text { of Economics, "Insecure Property Rights and } \\
\text { Government Ownership of Firms." May, } \\
\text { 1998. }\end{array}$ & Jiahua Che and Yingyi Qian & May 1997 \\
\hline $\begin{array}{l}\text { No. 51: Incentives, Scale Economies, and } \\
\text { Organizational Form }\end{array}$ & $\begin{array}{l}\text { Eric Maskin, Yingyi Qian, and Chenggang } \\
X u\end{array}$ & May 1997 \\
\hline $\begin{array}{l}\text { No. 50: Published in: Post-Soviet-Affairs, } \\
\text { "End of the Tunnel? The Effects of Financial } \\
\text { Stabilization in Russia" April-June 1997, } \\
\text { pages 105-33 }\end{array}$ & $\begin{array}{l}\text { Barry W. Ickes, Peter Murrell, and Randi } \\
\text { Ryterman }\end{array}$ & March 1997 \\
\hline $\begin{array}{l}\text { No. 49: The Evolution of Bank Credit Quality } \\
\text { in Transition: Theory and Evidence from } \\
\text { Romania }\end{array}$ & Enrico C. Perotti and Octavian Carare & October 1996 \\
\hline $\begin{array}{l}\text { No. 48: Where Do the Leaders Trade? } \\
\text { Information Revelation and Interactions } \\
\text { Between the Segments of Czech Capital } \\
\text { Markets }\end{array}$ & Jan Hanousek and Libor Nemecek & May 1997 \\
\hline $\begin{array}{l}\text { No. 47: Firms' Heterogeneity in Transition: } \\
\text { Evidence from a Polish Panel Data Set }\end{array}$ & Irena Grosfeld and Jean-François Nivet & May 1997 \\
\hline $\begin{array}{l}\text { No. 46: Strategic Creditor Passivity, } \\
\text { Regulation, and Bank Bailouts }\end{array}$ & Janet Mitchell & May 1997 \\
\hline $\begin{array}{l}\text { No. 45a: Decentralization in Transition } \\
\text { Economies: A Tragedy of the Commons? }\end{array}$ & Daniel M. Berkowitz and Wei Li & September 1997 \\
\hline $\begin{array}{l}\text { No. 44a: The Information Content of Stock } \\
\text { Markets: Why do Emerging Markets have } \\
\text { Synchronous Stock Price Movements? } \\
\text { (forthcoming in the Journal of Financial } \\
\text { Economics). } \\
\text { No. 43: Agency in Project Screening and } \\
\text { Termination Decisions: Why Is Good Money } \\
\text { Thrown After Bad? }\end{array}$ & $\begin{array}{l}\text { Randall Morck, Bernard Yeung, and } \\
\text { Wayne Yu }\end{array}$ & May 1997 \\
\hline $\begin{array}{l}\text { No. 42: Published in: Economics of } \\
\text { Transition, "Channels of Redistribution: }\end{array}$ & $\begin{array}{l}\text { Simon Commander, Andrei Tolstopiatenko, } \\
\text { and Ruslan Yemtsov }\end{array}$ & May 1997 \\
\hline
\end{tabular}

Publications denoted by an asterisk are not available on the Kresge Library webpage (http://www.wdi.bus.umich.edu) 


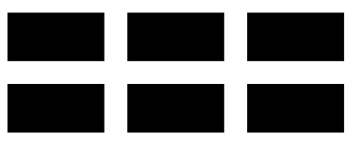

THE WILLIAM DAVIDSON INSTITUTE

AT THE UNIVERSITY OF MICHIGAN BUSINESSSCHOOL

\begin{tabular}{|c|c|c|}
\hline $\begin{array}{l}\text { Inequality and Poverty in the Russian } \\
\text { Transition." Vol. } 7 \text { (2) } 1999 .\end{array}$ & & \\
\hline $\begin{array}{l}\text { No. 41: Published in: Economics of } \\
\text { Transition, "Labour Market Characteristics } \\
\text { and Profitability: Econometric Analysis of } \\
\text { Hungarian Exporting Firms, 1986-1995" } \\
\text { 6(1), May 1998, pages 145-62 }\end{array}$ & László Halpern and Gabor Korosi & May 1997 \\
\hline $\begin{array}{l}\text { No. 40: Published in: the Harvard Law } \\
\text { Review, "The Tragedy of the Anticommons: } \\
\text { Property in the Transition from Marx to } \\
\text { Markets." January } 1998 .\end{array}$ & Michael Heller & February 1997 \\
\hline $\begin{array}{l}\text { No. 39: Privatization and Managerial } \\
\text { Efficiency }\end{array}$ & Olivier Debande and Guido Friebel & May 1997 \\
\hline $\begin{array}{l}\text { No. } 38 \text { Published in: The Quarterly Journal } \\
\text { of Economics, "Disorganization." Vol. 112, } \\
\text { No. 4, November 1997, pp. 1091-1126. }\end{array}$ & Olivier Blanchard and Michael Kremer & January 1997 \\
\hline $\begin{array}{l}\text { No. 37: Published in: Economics of } \\
\text { Transition, "Transition and the Output Fall." } \\
\text { 7(1), 1999, pages 1-28. }\end{array}$ & Gérard Roland and Thierry Verdier & March 1997 \\
\hline $\begin{array}{l}\text { No. 36: Restructuring an Industry During } \\
\text { Transition: A Two-Period Model }\end{array}$ & Richard Ericson & September 1996 \\
\hline $\begin{array}{l}\text { No. 34: The East-West Joint Venture: } B C \\
\text { Torsion Case Study }\end{array}$ & Sonia Ferencikova and Vern Terpstra & December 1998 \\
\hline $\begin{array}{l}\text { No. } 33 \text { Published in: Journal of Comparative } \\
\text { Economics, "Quantifying Price Liberalization } \\
\text { in Russia." Vol. 26, No. 4, December 1998, } \\
\text { pp. } 735-737 .\end{array}$ & $\begin{array}{l}\text { Daniel Berkowitz, David DeJong, and } \\
\text { Steven Husted }\end{array}$ & December 1998 \\
\hline $\begin{array}{l}\text { No. 32: What Can North Korea Learn from } \\
\text { China's Market Reforms? }\end{array}$ & John McMillan & September 1996 \\
\hline $\begin{array}{l}\text { No. 31: Published in : China-Economic- } \\
\text { Review, “Towards a Model of China as a } \\
\text { Partially Reformed Developing Economy } \\
\text { Under a Semifederalist Government.", 9(1), } \\
\text { Spring 1998, pages 1-23. }\end{array}$ & Yijiang Wang and Chun Chang & March 1997 \\
\hline $\begin{array}{l}\text { No. 30: Convergence in Output in Transition } \\
\text { Economies: Central and Eastern Europe, } \\
\text { 1970-1995 }\end{array}$ & Saul Estrin and Giovanni Urga & February 1997 \\
\hline $\begin{array}{l}\text { No. 29: Published in: Economics of } \\
\text { Transition, "Altered Band and Exchange } \\
\text { Volatility." Volume 6, no. 1, 1998, 173-181. }\end{array}$ & Evzen Kocenda & March 1997 \\
\hline $\begin{array}{l}\text { No. 28: Published in: Quarterly Journal of } \\
\text { Economics, "Public Versus Private } \\
\text { Ownership of Firms: Evidence from Rural } \\
\text { China." Volume 113, no. 3, August 1998, 773- } \\
\text { 808. }\end{array}$ & Hehui Jin and Yingyi Qian & January 1997 \\
\hline $\begin{array}{l}\text { No. 27: East-West Joint Ventures in a } \\
\text { Transitional Economy: The Case of Slovakia }\end{array}$ & Sonia Ferencikova & March 1997 \\
\hline $\begin{array}{l}\text { No. 26: Published in Economic Analysis } \\
\text { "Behavior of a Slovenian Firm in Transition" } \\
\text { Vol. 1, no. 1, 1998, 57-73. }\end{array}$ & Janez Prasnikar & February 1997 \\
\hline $\begin{array}{l}\text { No. 25: Cultural Encounters and Claims to } \\
\text { Expertise in Postcommunist Capitalism }\end{array}$ & Michael D. Kennedy & February 1997 \\
\hline
\end{tabular}

Publications denoted by an asterisk are not available on the Kresge Library webpage (http://www.wdi.bus.umich.edu) 


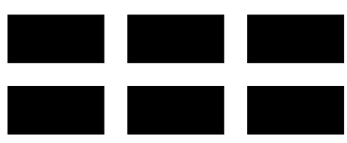

THE WILLIAM DAVIDSON INSTITUTE

AT THE UNIVERSITY OF MICHIGAN BUSINESSSCHOOL

\begin{tabular}{|c|c|c|}
\hline $\begin{array}{l}\text { No. 24: ZVU a.s.: Investment Funds on the } \\
\text { Board of Directors of an Engineering Giant }\end{array}$ & Tory Wolff & August 1995 \\
\hline $\begin{array}{l}\text { No. 23: The Role of Investment Funds in the } \\
\text { Czech Republic (joint publication with Czech } \\
\text { Management Center) }\end{array}$ & Dusan Triska & June 1996 \\
\hline $\begin{array}{l}\text { No. 22: Czech Investment Fund Industry: } \\
\text { Development and Behaviour (joint publication } \\
\text { with Czech Management Center) }\end{array}$ & Richard Podpiera & May 1996 \\
\hline $\begin{array}{l}\text { No. 21: Restructuring of Czech Firms: An } \\
\text { Example of Gama, a.s. (joint publication with } \\
\text { Czech Management Center) }\end{array}$ & Antonin Bulin & June 1996 \\
\hline $\begin{array}{l}\text { No. 20: YSE Funds: A Story of Czech } \\
\text { Investment Funds (joint publication with } \\
\text { Czech Management Center) }\end{array}$ & Michal Otradovec & November 1995 \\
\hline $\begin{array}{l}\text { No. 19: Prvni Investicni a.s., The First } \\
\text { Investment Corporation (joint publication } \\
\text { with Czech Management Center) }\end{array}$ & Jaroslav Jirasek & August 1995 \\
\hline $\begin{array}{l}\text { No. 18: PPF a.s., The First Private Investment } \\
\text { Fund (joint publication with Czech } \\
\text { Management Center) }\end{array}$ & Michal Otradovec & November 1995 \\
\hline $\begin{array}{l}\text { No. } 17 \text { Published in: Post-Soviet Geography } \\
\text { and Economics, "Russia's Managers in } \\
\text { Transition: Pilferers or Paladins?" Vol. } 37, \\
\text { o.7 (September 1996), pp. } 397-426 . \\
\end{array}$ & Susan J. Linz and Gary Krueger & November 1996 \\
\hline $\begin{array}{l}\text { No. 16: Banks in Transition-Investment } \\
\text { Opportunities in Central Europe and Russia } \\
\text { Edited Transcript from } 31 \text { May } 1996 \\
\text { Conference in New York City }\end{array}$ & $\begin{array}{l}\text { With commentary and edited by Anna } \\
\text { Meyendorff }\end{array}$ & January 1997 \\
\hline $\begin{array}{l}\text { No. 15: Marketing in Transitional Economies: } \\
\text { Edited Transcript \& Papers from } 1 \text { April } 1996 \\
\text { Conference in Ann Arbor, Michigan }\end{array}$ & Compiled by The Davidson Institute & December 1996 \\
\hline $\begin{array}{l}\text { No. 14: Pensions in the Former Soviet Bloc: } \\
\text { Problems and Solutions. Published by } \\
\text { Council on Foreign Relations. "The Coming } \\
\text { Global Pension Crisis" New York, } 1997 \\
\end{array}$ & Jan Svejnar & November 1996 \\
\hline $\begin{array}{l}\text { No. 13: Enterprise Restructuring and } \\
\text { Performance in the Transition. Forthcoming } \\
\text { in Financial Systems in Transition: The } \\
\text { Design of Financial Systems in Central } \\
\text { Europe eds. Anna Meyendorff and Anjan } \\
\text { Thakor. }\end{array}$ & $\begin{array}{l}\text { Lubomir Lizal, Miroslav Singer, and Jan } \\
\text { Svejnar }\end{array}$ & December 1996 \\
\hline $\begin{array}{l}\text { No. } 12 \text { Published in: Journal of International } \\
\text { Marketing, "Executive Insights: Marketing } \\
\text { Issues and Challenges in Transitional } \\
\text { Economies." Vol. 5, No. 4, 1997, pp. 95-114. } \\
\text { Also published in: Marketing Issues in } \\
\text { Transitional Economies ed. Rajeev Batra. }\end{array}$ & Rajeev Batra & April 1997 \\
\hline $\begin{array}{l}\text { No. 11: Worker Trust and System } \\
\text { Vulnerability in the Transition from Socialism } \\
\text { to Capitalism }\end{array}$ & Andrew Schotter & August 1996 \\
\hline $\begin{array}{l}\text { No. } 10 \text { Published in: Comparative Economic } \\
\text { Studies, "Russian Firms in Transition: }\end{array}$ & Susan J. Linz & July 1996 \\
\hline
\end{tabular}

Publications denoted by an asterisk are not available on the Kresge Library webpage (http://www.wdi.bus.umich.edu) 


\section{$\square \square$ \\ THE WILLIAM DAVIDSON INSTITUTE \\ AT THE UNIVERSITY OF MICHIGAN BUSINESSSCHOOL}

\begin{tabular}{|c|c|c|}
\hline $\begin{array}{l}\text { Champions, Challengers, and Chaff." Vol. } \\
\text { 39, No.2, Summer 1997, pp. 1-36. }\end{array}$ & & \\
\hline $\begin{array}{l}\text { No. 9: Corporate Debt Crisis and Bankruptcy } \\
\text { Law During the Transition: The Case of China }\end{array}$ & David D. Li and Shan Li & December 1995 \\
\hline $\begin{array}{l}\text { No. } 8 \text { Published in: Journal of Comparative } \\
\text { Economics, "A Theory of Ambiguous } \\
\text { Property Rights in Transition Economies: The } \\
\text { Case of the Chinese Non-State Sector." Vol. } \\
\text { 23, No. 1, August 1996, pp. 1-19. }\end{array}$ & David D. Li & June 1996 \\
\hline $\begin{array}{l}\text { No. 7: The Foreign Economic Contract Law of } \\
\text { China: Cases and Analysis }\end{array}$ & Dong-lai Li & June 1993 \\
\hline $\begin{array}{l}\text { No. 3: Bank Privatization in Hungary and the } \\
\text { Magyar Kulkereskedelmi Bank Transaction }\end{array}$ & Roger Kormendi and Karen Schnatterly & May 1996 \\
\hline $\begin{array}{l}\text { Replacing Nos. 1-2 \& 4-6: Journal of } \\
\text { Comparative Economics Symposium on } \\
\text { "Bank Privatization in Central Europe and } \\
\text { Russia." Vol. 25, No. 1, August } 1997 .\end{array}$ & $\begin{array}{l}\text { No. } 1 \text { "Bank Privatization in Transitional } \\
\text { Economies" by Roger Kormendi and } \\
\text { Edward Snyder. No. } 2 \text { "Transactional } \\
\text { Structures of Bank Privatizations in } \\
\text { Central Europe and Russia" by Anna } \\
\text { Meyendorff and Edward A. Snyder. No. } 4 \\
\text { "Bank Privatization in Poland: The Case } \\
\text { of Bank Slaski" by Jeffery Abarbaness and } \\
\text { John Bonin. No. } 5 \text { "Bank Privatization in } \\
\text { Post-Communist Russia: The Case of } \\
\text { Zhilsotsbank" by Jeffery Abarbanell and } \\
\text { Anna Meyendorff and No. 6 "'The Czech } \\
\text { Republic's Commercial Bank: Komercni } \\
\text { Banka" by Edward A. Snyder and Roger } \\
\text { C. Kormendi. }\end{array}$ & August 1997 \\
\hline
\end{tabular}

Publications denoted by an asterisk are not available on the Kresge Library webpage (http://www.wdi.bus.umich.edu) 